\title{
Rudimentos de Mecânica, Ações Hamiltoneanas e Aplicação Momento
}

\author{
Guilherme Casas Gonçalves
}

\author{
DISSERTAÇÃO APRESENTADA \\ $\mathrm{AO}$ \\ Instituto De Matemática e EstatísticA \\ DA \\ Universidade DE SÃo PAUlo \\ PARA \\ OBTENÇÃO DO TÍTULO \\ $\mathrm{DE}$ \\ Mestre em CIÊNCIAS
}

Programa: Matemática

Orientador: Prof. Dr. Marcos Martins Alexandrino da silva

Durante o desenvolvimento deste trabalho o autor recebeu auxlio financeiro da CAPES/CNPq

São Paulo, maio de 2015 


\section{Rudimentos de Mecânica, Ações Hamiltoneanas e Aplicação Momento}

Esta versão da dissertação contém as correções e alterações sugeridas pela Comisso Julgadora durante a defesa da versão original do trabalho, realizada em 15/05/2015. Uma cópia da versão original está disponível no

Instituto de Matemática e Estatística da Universidade de São Paulo.

Comisso Julgadora:

- Prof. Dr. Marcos Martins Alexandrino da Silva (orientador) - IME-USP

- Prof. Dr. Dirk Töben - UFSCAR

- Prof. Dr. Miguel Domínguez Vásquez - IMPA 


\section{Resumo}

GONÇAlVES, G. C. Rudimentos de Mecânica, Ações Hamiltoneanas e Aplicação Momento. 2015. 120 f. Dissertação (Mestrado) - Instituto de Matemática e Estatística, Universidade de São Paulo, So Paulo, 2015.

Essa dissertação trata de geometria simplética e suas aplicações, apresentando conceitos tais como o gradiente simplético e também o teorema de Darboux. Discutimos a formulação Lagrangeana da mecânica, apresentando as equações de Euler-Lagrange e, usando a geometria simplética, mostramos como estes naturalmente evoluem para o formalismo Hamiltoneano e as equações de Hamilton. Introduzimos também o conceito da métrica de Jacobi e demonstramos o teorema de Noether. Apresentamos o conceito de ações simpléticas e Hamiltoneanas, bem como aplicações momento e comomento. São demonstrados resultados importantes como o teorema de KirillovKostant-Sourieau para órbitas coadjuntas e a redução simplética de Marsden-Weinstein-Meyer. Os resultados centrais apresentados são o teorema de Atiyah-Guillemin-Steinberg de convexidade, o teorema de Schur e Horn para matrizes unitárias e o teorema de Delzant, este último sendo apresentado apenas com uma idéia da prova.

Palavras-chave: ações, aplicação momento, mecânica. 


\section{Abstract}

GONÇAlVES, G. C. Rudiments of Mechanics, Hamiltonian Actions and Momentum Map. 2015. 120 f. Dissertação (Mestrado) - Instituto de Matemática e Estatística, Universidade de São Paulo, So Paulo, 2010.

This thesis is about symplectic geometry and its applications, presenting concepts such as the symplectic gradient and also Darboux's theorem. We discuss the Lagrangian formulation of mechanics, presenting the Euler-Lagrange equations and, using symplectic geometry, show how those naturally evolve into the Hamiltonian formalism and the Hamilton equations. We instroduce also the concept of the Jacobi metrics and prove Noether's theorem. We also introduce the concept of symplectic and Hamiltonian actions as well as moment and comoment maps. We prove important results such as the Kirillov-Kostant-Sourieau theorem for coadjoint orbits and the symplectic reduction of Marsden-Weinstein-Meyer. The central results presented are the convexity theorem of Guillemin-Atiyah-Steinberg, the Schur and Horn theorem for unitary matrices and the Delzant theorem, this last one being presented only with an idea of the proof.

Keywords: actions, mechanics, momentum map. 


\section{Sumário}

Lista de Símbolos $\quad$ vii

$\begin{array}{lc}\text { Lista de Figuras } & \text { ix }\end{array}$

1 Mecânica e Rudimentos de Geometria Simplética 3

1.1 Formulação Lagrangeana . . . . . . . . . . . . . . . . . . . . . . . . . . . . 3

1.1 .1 Equações de Euler-Lagrange . . . . . . . . . . . . . . . . . . . . . . . . . . . . . . .

1.1 .2 Equações de Newton . . . . . . . . . . . . . . . . . . . . . . . . . . . . 7

1.1.3 Transformação de Legendre e Energia . . . . . . . . . . . . . . . . . . . . 8

1.1.4 Teorema de Noether . . . . . . . . . . . . . . . . . . . . . . . . . 10

1.2 Formulação Hamiltoneana . . . . . . . . . . . . . . . . . . . . . . . . . 12

1.2.1 Rudimentos de Geometria Simplética . . . . . . . . . . . . . . . . . . . 12

1.2 .2 Equações de Hamilton . . . . . . . . . . . . . . . . . . . . . . . . 17

1.2 .3 Métrica de Jacobi . . . . . . . . . . . . . . . . . . . . . . . . . . . . . . . . . 21

2 Fatos Básicos e Ações simpléticas $\quad 25$

2.1 Introdução . . . . . . . . . . . . . . . . . . . . . . . . 25

2.2 Ações Diferenciáveis . . . . . . . . . . . . . . . . . . . . . . . 25

2.3 Ações Simpléticas, Hamiltonianas e Aplicação Momento . . . . . . . . . . . . . . . 26

2.4 Teorema de Kirillov-Kostant-Sourieau . . . . . . . . . . . . . . . . . . . . 36

2.5 Redução Simplética . . . . . . . . . . . . . . . . . . . . . . . . . . . 39

2.5 .1 Resultados preliminares . . . . . . . . . . . . . . . . . . 39

2.5.2 Teorema de Marsden-Weinstein-Meyer . . . . . . . . . . . . . . . . . . 40

2.5.3 Redução para Grupos Produto . . . . . . . . . . . . . . . . . . . . . . . . 42

2.6 Condições para Existência e para a Unicidade da Aplicação Comomento . . . . . . . 43

2.6.1 Existência da Aplicação Comomento . . . . . . . . . . . . . . . . . . . . . . 44

2.6.2 Unicidade da Aplicação Comomento . . . . . . . . . . . . . . . . . . . 45

3 Aplicação Momento e Convexidade $\quad 47$

3.1 Introdução . . . . . . . . . . . . . . . . . . . . . . . . . . . . 47

3.2 Teorema de Convexidade . . . . . . . . . . . . . . . . . . . . . 48

3.2.1 Estruturas Quase-Complexas . . . . . . . . . . . . . . . . . 48

3.2 .2 Teoria de Morse . . . . . . . . . . . . . . . . . . . . . . . . 49

3.2 .3 Prova do Teorema de Convexidade . . . . . . . . . . . . . . . . . . . . 51

3.2 .4 Prova dos Lemas . . . . . . . . . . . . . . . . . . . . . . . . 55 
3.3 Teorema de Schur e Horn . . . . . . . . . . . . . . . . . . . . . 60

3.4 Teorema de Delzant . . . . . . . . . . . . . . . . . . . 63

$\begin{array}{ll}\text { Referências Bibliográficas } & 69\end{array}$ 


\title{
Lista de Símbolos
}

\author{
$\psi \quad$ Ação suave \\ $\mu \quad$ Aplicação momento \\ $\omega \quad$ Forma simplética \\ $G \quad$ Grupo de Lie \\ $\mathfrak{g} \quad$ Álgebra de Lie de $G$ \\ $T^{n} \quad$ Toro n-dimensional $=S^{1} \times \ldots \times S^{1}$ \\ $\mathfrak{t} \quad$ Álgebra de Lie de um toro \\ $\eta \quad$ Elemento de $\mathfrak{g}$ \\ $\xi, \zeta \quad$ Elementos de $\mathfrak{g}^{*}$ \\ $M \quad$ Variedade diferenciável \\ $\mathfrak{X}(M) \quad$ Conjunto dos campos em $M$
}




\section{Lista de Figuras}

1.1 Diagrama transformação de Legendre e coordenadas . . . . . . . . . . . . . . . . . 9

1.2 Diagrama transformação de Legendre e coordenadas . . . . . . . . . . . . . . . . 20

3.1 Aproximando a reta $r$ por uma reta $\tilde{r}$ que intercepta $r^{\perp}$ em um ponto de $E_{\pi \circ \mu} \ldots \ldots 4$

3.2 Exemplos de politopos de Delzant . . . . . . . . . . . . . . . . . . . 63

3.3 Exemplos de politopos não Delzant . . . . . . . . . . . . . . . . . 63 


\section{Introdução}

Uma forma simplética é uma 2-forma fechada e não-degenerada, e uma variedade simplética é um $\operatorname{par}(M, \omega)$ onde $M$ é uma variedade e $\omega$ é uma forma simplética em $M$. Como a forma simplética é não-degenerada, para todo funcional $H: M \rightarrow \mathbb{R}$, chamado Hamiltoneano, podemos associar um campo $X_{H}$ com a propriedade $\mathrm{d} H=\omega\left(X_{H}, \cdot\right)$. Chamamos $X_{H}$ de gradiente simplético ou campo Hamiltoneano associado ao Hamiltoneano $H$. esse conceito é muito útil em certos formalismos de mecânica, onde o fibrado cotangente, que é um exemplo de variedade simplética, é usado para representar o espaço posição-momento.

Uma ação $\psi$ de um grupo de Lie $G$ numa variedade simplética $M$ é dita simplética se ela preserva a forma simplética $\omega$. Sabemos que cada elemento $\eta$ de $\mathfrak{g}$ álgebra de Lie de $G$ induz um campo $X^{\eta}$. É possível mostrar (vide proposição 2.3.2) que para uma ação simplética, esses campos são localmente Hamiltoneanos. Se isto for válido globalmente a ação será chamada fracamente Hamiltoneana. Neste caso podemos definir uma aplicação linear $\mu^{*}: \mathfrak{g} \rightarrow C^{\infty}(M)$ que a cada $\eta$ elemento da álgebra de Lie associa uma função $\mu^{*}(\eta)$ tal que o campo Hamiltoneano $X_{\mu^{*}(\eta)}$ associado a ela coincide com o campo $X^{\eta}$. Se além disso $\mu^{*}$ for um homomorfismo de Lie (entre o colchete de Lie e o colchete de Poisson) $\mu^{*}$ será chamada aplicação comomento e a ação será dita Hamiltoneana (vide definição 2.3.4). Finalmente, se invertermos a ordem dos argumentos dessa função, podemos definir a aplicação momento $\mu: M \rightarrow \mathfrak{g}^{*}$ por $\mu(p)(\eta)=\mu^{*}(\eta)(p)$. Como veremos no capitulo 2 o momento linear e o momento angular são exemplos de aplicação momento.

Um dos resultados centrais desta tese será teorema de convexidade de Atiyah-Guillemin-Steinberg que garante entre outras coisas, que a imagem da aplicação momento associada à ação de um toro é um conjunto convexo (vide teorema 3.1.1). Discutiremos aqui a prova de Atiyah [8], baseado em [3] e [5]. Um outro teorema, chamado teorema de Delzant [9], afirma que esse conjunto não só é convexo, mas pertence à classe especial dos politopos de Delzant e está associado univocamente a uma única variedade simplética tórica. Apresentaremos na seção 3.4 uma idéia da prova desse teorema, baseado em [1]. Um outro teorema importante apresentado, que é um corolário do teorema de convexidade é o teorema de Shur e Horn, que afirma que a diagonal de uma matriz unitária está no casco convexo das permutações do seu espectro (vide 3.1.2)

Agora discutiremos o conteúdo da dissertação por capítulo. O capítulo 1 mostra uma aplicação da geometria simplética, em particular seu papel em desenvolver um formalismo para a mecânica. Na seção 1.1 apresentamos o formalismo Lagrangeano, definindo o funcional ação e derivando as equações de Euler-Lagrange na seção 1.1.1, tanto para $\mathbb{R}^{n}$ quanto para variedades. Em seguida mostramos na seção 1.1.2 como esse formalismo realmente reflete a mecânica de Newton e na seção 1.1.3 apresentando os conceitos de Transformação de Legendre e funcional energia que serão necessários mais adiante. Encerramos a seção com a prova do teorema de Noether e um exemplo de sua aplicação em 1.1.4.

A seção 1.2 começa com uma introdução à geometria simplética na seção 1.2 .1 começando com formas simpléticas e estruturas lineares simpléticas, espaços vetoriais e variedades simpléticas, seguidos de exemplos. Em seguida apresentamos o teorema de Darboux, que diz que localmente toda variedade simplética é equivalente ao $\mathbb{R}^{2 n}$ munido de sua forma simplética canônica. Introduzimos também conceitos tais como o gradiente simplético e o complementar simplético que serão usados mais à frente, por exemplo, na definição de aplicação momento e no teorema de redução de Marsden, respectivamente. Na seção 1.2.2 definimos as equações de Hamilton no fibrado cotangente e, com a transformação de Legendre, mostramos que, para certos Lagrangeanos, podemos escolher funções 
Hamiltoneanas tais que suas respectivas equações de Euler-Lagrange e de Hamilton sejam equivalentes sob o isomorfismo da transformação de Legendre. Encerramos o capítulo com a seção 1.2.3 apresentando a prova do teorema da métrica de Jacobi que apresenta, para certos Lagrangeanos $L$, uma métrica cujas geodésicas equivalem às soluções das equações de Euler-Lagrange para $L$.

No capítulo 2, começamos com uma revisão de ações diferenciáveis na seção 2.2 , revendo os conceitos de ação infinitesimal, ações adjunta e coadjunta de um grupo de Lie e equivariância. Na seção 2.3 introduzimos o conceito de ações simpléticas e Hamiltoneanas, apresentando as aplicações momento e comomento e a relação entre as duas. Apresentamos também alguns lemas que nos auxiliam a criar novas ações Hamiltoneanas a partir de ações Hamiltoneanas existentes, bem como exemplos de tais ações, alguns dos quais serão usados no capítulo 3. Na seção 2.4 demonstramos o teorema de Kirillov-Kostant-Sourieau que diz que toda órbita coadjunta é uma variedade simplética e que a ação coadjunta restrita a essa órbita é Hamiltoneana. Esse teorema será usado na seção 3.3 para provar o teorema de Schur e Horn. A seção 2.5 é dedicada a provar o teorema de MarsdenWeinstein-Meyer de redução simplética que nos permite usar uma ação Hamiltoneana para reduzir uma variedade preservando a forma simplética e também como fazer uma "redução parcial"quando temos um grupo produto, fazendo a redução com uma componente e induzindo uma ação da outra componente no quociente (tais ideias serão utilizadas na ideia da prova do teorema de Delzant, seção 3.4).

O capítulo 3 contém os resultados principais da dissertação. Começamos pelo teorema de convexidade de Atiyah-Guillemin-Steinberg na seção 3.2. Para provar esse teorema introduzimos na seção 3.2.1 o conceito de estruturas triplas, uma estrutura quase-complexa, uma métrica Riemanniana e uma forma simplética compatíveis em algum sentido, e na seção 3.2.2 apresentamos funções de Morse-Bott, que são funções cujos pontos críticos formam subvariedades cujo espaço tangente coincide com o kernel de sua Hessiana. Combinando esses dois elementos apresentamos na seção 3.2.3 a demonstração do teorema de convexidade. Nas seções 3.2.2 e 3.2.3 são enunciados lemas sem prova a fim de não desviar a atenção do leitor do foco principal. As provas desses lemas são feitas na seção 3.2.4. Em seguida, na seção 3.3, apresentamos um corolário do teorema de convexidade, o teorema de Schur e Horn para matrizes unitárias. O último teorema apresentado é o teorema de Delzant, na seção 3.4. Por ser um teorema com muitos aspectos técnicos, apresentamos aqui apenas uma idéia da prova, fazendo um caso específico de dimensão baixa e, ao final, explicando como se faria a prova para um caso de dimensão maior. 


\section{Capítulo 1}

\section{Mecânica e Rudimentos de Geometria Simplética}

Após a modelagem original de Newton outros formalismos surgiram para lidar com o mesmo problema por outras abordagens. Esses formalismos não são meras formas de reescrever a mecânica clássica de Newton. Eles já foram adaptados a outros problemas em áreas aparentemente não relacionadas, como a economia e a biologia.

Neste capítulo serão apresentados dois formalismos. O Lagrangeano se baseia em encontrar pontos críticos de funcionais, buscando curvas que o minimizem. Já o Hamiltoneano usa fundamentos de geometria simplética para encontrar curvas que preservem alguma quantidade.

Esse capítulo é baseado em [4].

\subsection{Formulação Lagrangeana}

A formulação Lagrangeana se baseia em encontrar pontos críticos de um funcional cujo domínio será um espaço de curvas. Veremos que tais curvas minimizam uma grandeza ao longo delas, e que elas estão intimamente relacionadas a geodésicas da geometria Riemanniana. Na verdade, veremos que, para certos Lagrangeanos, podemos encontrar uma métrica (chamada métrica de Jacobi) tal que as curvas críticas do funcional sejam geodésicas.

\subsubsection{Equações de Euler-Lagrange}

Seja $M$ variedade Riemanniana e $L: T M \rightarrow \mathbb{R}$ função suave que chamaremos Lagrangeano. Dados $x_{0}, x_{1} \in M$, defina $C_{x_{0}}^{x_{1}}(M)$ como o conjunto das curvas $\alpha:[0,1] \rightarrow M$ suaves por partes $\operatorname{com} \alpha(0)=x_{0}$ e $\alpha(1)=x_{1}$. Então o funcional ação associado a $L$ é a função $I_{L}: C_{x_{0}}^{x_{1}}(M) \rightarrow \mathbb{R}$ dada por

$$
I_{L}(\alpha)=\int_{0}^{1} L\left(\alpha^{\prime}(t)\right) \mathrm{d} t
$$

A formulação Lagrangeana se baseia no princípio da ação minima: as soluções do sistema são aquelas que minimizam o funcional ação associado ao Lagrangeano. Queremos então buscar pontos críticos de $I_{L}$ para encontrar curvas que minimizem esse funcional. Para isso, no entanto, precisamos antes definir um objeto chamado variação.

Definição 1.1.1. Considere uma curva $\alpha \in C_{x_{0}}^{x_{1}}(M)$ e pontos $0=t_{0}<\ldots<t_{n}=1$ tais que $\left.\alpha\right|_{\left(t_{i}, t_{i+1}\right)}$ é suave para todo $i \in\{0, \ldots, n\}$. Uma variação de $\alpha$ é uma função suave por partes $h$ : $[0,1] \times(-\epsilon, \epsilon) \rightarrow M$, com $\epsilon>0$, tal que $h^{i}=\left.h\right|_{\left[t_{i}, t_{i+1}\right] \times(-\epsilon, \epsilon)}$ é suave e $h_{0}=\alpha$, onde $h_{s}(t)=h(s, t)$. Dizemos que a variação é própria se $h_{s}(0)=\alpha(0)$ e $h_{s}(1)=\alpha(1)$ para todo $s \in[0,1]$.

Agora podemos definir que uma curva $\alpha \in C_{x_{0}}^{x_{1}}(M)$ é ponto crítico de $I_{L}$ se $\left.\frac{\mathrm{d}}{\mathrm{d} s} I_{L}\left(h_{s}\right)\right|_{s=0}=0$ para toda variação $h$ de $\alpha$. 
No lema 1.1 .3 adiante, dada uma variação $h$ de uma curva $\alpha \in C_{x_{0}}^{x_{1}}(M)$ iremos querer calcular $\frac{\partial h}{\partial s}$. Vamos ver que isso sempre será possível. Da definição é óbvio que $\frac{\partial h_{s}}{\partial s}(t)$ existe para todo $t$ em que $\alpha$ é suave. Para ver que a derivada parcial existe num $t_{i}$ onde $\alpha$ não é derivável, recorde que $\left.h\right|_{\left[t_{i}, t_{i+1}\right] \times(-\epsilon, \epsilon)}$ ser suave é equivalente a encontrar $\delta>0$ e extensão $\tilde{h}^{i}:\left(t_{i}-\delta, t_{i+1}+\delta\right) \times(-\epsilon, \epsilon) \rightarrow M$ suave de $h_{i}$. Logo, para cada $t_{i}$ temos duas maneiras de calcular $\frac{\partial h_{s}}{\partial s}\left(t_{i}\right)$, usando $\tilde{h}^{i-1}$ ou $\tilde{h}^{i}$. Mas, note que $\tilde{h}_{s}^{i-1}\left(t_{i}\right)=\tilde{h}^{i}\left(t_{i}\right)$ pela continuidade de $h$, logo $\frac{\partial h_{s}}{\partial s}\left(t_{i}\right)$ está unicamente definido.

Antes de prosseguir, apresentaremos um lema que será útil nas próximas passagens, chamado lema fundamental do cálculo das variações.

Lema 1.1.2. Seja $f=\left(f_{1}, \ldots, f_{n}\right): \mathbb{R}^{n} \rightarrow \mathbb{R}^{m}$ suave tal que $\int_{0}^{1} \sum_{i=0}^{n} f_{i}(t) \delta_{i}(t) \mathrm{d} t=0$, para toda função $\delta=\left(\delta_{1}, \ldots, \delta_{n}\right): \mathbb{R}^{n} \rightarrow \mathbb{R}^{m}$ suave com $\delta(0)=\delta(1)=0$ para todo inteiro $n \geq 0$. Então $f=0$.

Seria interessante obter uma maneira mais eficiente de determinar pontos críticos de $I_{L}$. O lema a seguir mostra uma maneira para o caso $M=\mathbb{R}^{m}$. Veremos depois que a condição pode ser extendida ao caso geral. Apenas para esclarecer a notação utilizada, trataremos o Lagrangeano $L$ como uma função com domínio em $\mathbb{R}^{2 n}$. As primeiras $m$ coordenadas serão chamadas $q_{1}, \ldots, q_{m} \mathrm{e}$ as $m$ últimas, $\dot{q}_{1}, \ldots, \dot{q}_{m}$.

Note também os diferentes usos do sinal $(\cdot)^{\prime}$ para curvas. Dada uma curva $\alpha:[a, b] \rightarrow M$, se $M$ é uma variedade diferenciável arbitrária, $\alpha^{\prime}$ é uma curva no fibrado tangente T $M$. No caso $M=\mathbb{R}^{n}$, por outro lado, a mesma notação $\alpha^{\prime}$ denota uma nova curva com contra-domínio $\mathbb{R}^{n}$, enquanto a curva no fibrado tangente $\mathrm{TR}^{n}=\mathbb{R}^{n} \times \mathbb{R}^{n}$ é dada por $\left(\alpha, \alpha^{\prime}\right)$. Ambas as notações serão usadas e a diferença deverá ficar clara pelo contexto.

Lema 1.1.3 (Equações de Euler-Lagrange). Sejam $L: \mathbb{R}^{m} \times \mathbb{R}^{m} \rightarrow \mathbb{R}$ um Lagrangeano, $x_{0}, x_{1} \in \mathbb{R}^{m}$ e $\alpha \in C_{x_{0}}^{x_{1}}$. Então $\alpha$ é ponto crítico de $I_{L}$ se, e somente se, valem as chamadas equações de EulerLagrange

$$
\frac{\partial L}{\partial q_{i}}\left(\alpha(t), \alpha^{\prime}(t)\right)=\frac{\mathrm{d}}{\mathrm{d} t} \frac{\partial L}{\partial \dot{q}_{i}}\left(\alpha(t), \alpha^{\prime}(t)\right)
$$

Diremos então que $\alpha$ é solução de Euler-Lagrange para L, ou simplesmente solução de EulerLagrange, quando o Lagrangeano em questão estiver claro.

Demonstração. Considere $h$ variação própria de $\alpha$, e chame $\delta=\left.\frac{\partial h_{s}}{\partial s}\right|_{s=0}$. Então

$$
\left.\frac{\mathrm{d}}{\mathrm{d} s} I_{L}\left(h_{s}\right)\right|_{s=0}=\left.\int_{0}^{1} \frac{\partial}{\partial s} L\left(h_{s}(t),\left(h_{s}\right)^{\prime}(t)\right)\right|_{s=0} \mathrm{~d} t
$$

$\log \mathrm{O}$

$$
\left.\frac{\mathrm{d}}{\mathrm{d} s} I_{L}\left(h_{s}\right)\right|_{s=0}=\int_{0}^{1} \sum_{i=1}^{n} \frac{\partial}{\partial q_{i}} L\left(\alpha(t), \alpha^{\prime}(t)\right) \delta_{i}(t) \mathrm{d} t+\int_{0}^{1} \sum_{i=1}^{n} \frac{\partial}{\partial \dot{q}_{i}} L\left(\alpha(t), \alpha^{\prime}(t)\right) \delta_{i}^{\prime}(t) \mathrm{d} t
$$

Considere a segunda integral. Usando integração por partes, temos que

$\int_{0}^{1} \sum_{i=1}^{n} \frac{\partial}{\partial \dot{q}_{i}} L\left(\alpha(t), \alpha^{\prime}(t)\right) \delta_{i}^{\prime}(t) \mathrm{d} t=\left.\sum_{i=1}^{n} \frac{\partial}{\partial \dot{q}_{i}} L\left(\alpha(t), \alpha^{\prime}(t)\right) \delta_{i}(t)\right|_{t=0} ^{t=1}-\int_{0}^{1} \sum_{i=1}^{n} \frac{\mathrm{d}}{\mathrm{d} t} \frac{\partial}{\partial \dot{q}_{i}} L\left(\alpha(t), \alpha^{\prime}(t)\right) \delta_{i}(t) \mathrm{d} t$

Como $h$ é própria, temos que $\delta(0)=\delta(1)=0$. Logo, temos que a primeira parcela do termo à direita é zero. Assim, substituindo (1.3) em (1.2) e colocando $\delta_{i}$ em evidência, temos

$$
\left.\frac{\mathrm{d}}{\mathrm{d} s} I_{L}\left(h_{s}\right)\right|_{s=0}=\int_{0}^{1} \sum_{i=1}^{n}\left(\frac{\partial}{\partial q_{i}} L\left(\alpha(t), \alpha^{\prime}(t)\right)-\frac{\mathrm{d}}{\mathrm{d} t} \frac{\partial}{\partial \dot{q}_{i}} L\left(\alpha(t), \alpha^{\prime}(t)\right)\right) \delta(t) \mathrm{d} t
$$

Assuma primeiro que $\alpha$ é solução de Euler-Lagrange. Então $\left.\frac{\mathrm{d}}{\mathrm{d} s} I_{L}\left(h_{s}\right)\right|_{s=0}=0$ para toda variação 
$h$ de $\alpha$. Por outro lado assuma que $\alpha$ é ponto crítico de $I_{L}$. Então temos que o termo à direita da igualdade é zero. O resultado segue pelo lema 1.1.2.

Podemos definir soluções de Euler-Lagrange também para uma variedade Riemanniana $M$ arbitrária.

Definição 1.1.4. Considere $M$ variedade diferenciável e uma curva $\alpha:[0,1] \rightarrow M$. Dizemos que $\alpha$ é solução de Euler-Lagrange em coordenadas para um Lagrangeano $L: \mathrm{T} M \rightarrow \mathbb{R}$ no ponto $t_{0} \in[0,1]$ se para toda carta $\psi: U \subset \mathbb{R}^{m} \rightarrow V \subset M$ em $M$ com $\alpha\left(t_{0}\right) \in V$ temos que

$$
\frac{\partial L_{\psi}}{\partial q_{i}}\left(\psi^{-1} \circ \alpha\left(t_{0}\right),\left(\psi^{-1} \circ \alpha\right)^{\prime}\left(t_{0}\right)\right)=\left.\frac{\mathrm{d}}{\mathrm{d} t} \frac{\partial L_{\psi}}{\partial \dot{q}_{i}}\left(\psi^{-1} \circ \alpha(t),\left(\psi^{-1} \circ \alpha\right)^{\prime}(t)\right)\right|_{t=t_{0}}
$$

onde $L_{\psi}=L \circ \mathrm{d} \psi$. Dizemos que $\alpha$ é solução de Euler-Lagrange em coordenadas para $L$ se $\alpha$ é solução de Euler-Lagrange em coordenadas segundo $L$ em todo $t \in[0,1]$. Nos dois casos poderemos omitir L quando este estiver claro.

O lema a seguir mostra que a definição 1.1.4 tem o mesmo sentido de minimizar um funcional.

Lema 1.1.5. Sejam $M$ variedade diferenciável e $L: \mathrm{T} M \rightarrow \mathbb{R}$ um Lagrangeano. Considere uma curva $\alpha:[0,1] \rightarrow M$ tal que existe $\psi: U \subset \mathbb{R} \rightarrow V \subset M$ carta de $M$ com $\alpha([0,1]) \subset V$. Então $\alpha$ é de Euler-Lagrange em coordenadas segundo L se, e só se,

$$
\left.\frac{\mathrm{d}}{\mathrm{d} s} I_{L}\left(h_{s}\right)\right|_{s=0}=0
$$

para toda $h:(-\epsilon, \epsilon) \times[0,1] \rightarrow M$ variação de $\alpha \operatorname{com} h((-\epsilon, \epsilon) \times[0,1]) \subset V$, onde $I_{L}$ é definido pela fórmula 1.1.

Demonstração. Se $h:(-\epsilon, \epsilon) \times[0,1] \rightarrow M$ é como descrita no enunciado, temos que

$$
\begin{aligned}
\left.\frac{\mathrm{d}}{\mathrm{d} s} I_{L}\left(h_{s}\right)\right|_{s=0} & =\int_{0}^{1} L\left(h_{s}(t)\right) \mathrm{d} t \\
& =\int_{0}^{1} L_{\psi}\left(\psi^{-1} \circ h_{s}(t)\right) \mathrm{d} t \\
& =\left.\frac{\mathrm{d}}{\mathrm{d} s} I_{L_{\psi}}\left(\psi^{-1} \circ h_{s}\right)\right|_{s=0}
\end{aligned}
$$

Nosso objetivo aqui é obter o resultado aplicando a proposição 1.1.3. Mas observe que nem toda variação de $\psi^{-1} \circ \alpha$ pode ser escrita como $\psi^{-1} \circ h$, com $h$ alguma variação de $\alpha$ com imagem contida em $V$. Podemos, no entanto, contornar esse problema argumentando que, para toda variação $g:(-\epsilon, \epsilon) \times[0,1] \rightarrow \mathbb{R}^{n}$ de $\psi^{-1} \circ \alpha$, podemos encontrar $\tilde{\epsilon}<\epsilon$ tal que $\tilde{g}=\left.g\right|_{(-\tilde{\epsilon}, \tilde{\epsilon}) \times[0,1]}=\psi^{-1} \circ h$ para alguma variação $h$ de $\alpha$ com imagem contida em $V$. E como

$$
\begin{aligned}
\left.\frac{\mathrm{d}}{\mathrm{d} s} I_{L_{\psi}}\left(g_{s}\right)\right|_{s=0} & =\left.\frac{\mathrm{d}}{\mathrm{d} s} I_{L_{\psi}}\left(\tilde{g}_{s}\right)\right|_{s=0} \\
& =\left.\frac{\mathrm{d}}{\mathrm{d} s} I_{L_{\psi}}\left(\psi^{-1} \circ h_{s}\right)\right|_{s=0}
\end{aligned}
$$

o resultado então segue da proposição 1.1.3

Note que o lema 1.1.5 implica, ao menos num caso particular que as equações de Euler-Lagrange independem de coordenadas, já que são equivalentes a um conceito intrínsico. Isso na verdade é um caso particular de um fato mais geral, o de as equações de Euler-Lagrange serem preservadas por difeomorfismos, como as cartas de uma variedade. Esse fato é enunciado formalmente na proposição a seguir. 
Proposição 1.1.6. Sejam $M, N$ variedades diferenciáveis e $f: M \rightarrow N$ um difeomorfismo. Se $L: \mathrm{T} M \rightarrow \mathbb{R}$ é um Lagrangeano então uma curva $\alpha:(-\epsilon, \epsilon) \rightarrow M$ é de Euler-Lagrange em coordenadas segundo $L$ se, e só se, $f^{-1} \circ \alpha$ é de Euler-Lagrange segundo $L_{f}$.

A prova é idêntica à do lema 1.1.5, trocando $\mathbb{R}^{n}$ por $N$ e $\psi$ por $f$.

Vamos agora tentar generalizar o lema 1.1.5 para curvas quaisquer. Começaremos provando um seguinte lema, mas para isso precisaremos introduzir uma nova notação. Ao considerar o funcional ação, sempre fixamos dois pontos $x_{0}, x_{1} \in M$ como os extremos das curvas consideradas. No lema a seguir, dados pontos $\tilde{x_{0}}$ e $\tilde{x}_{1}$ usaremos usaremos a notação $I_{L}^{\tilde{x}_{0}, \tilde{x}_{1}}$ para denotar o funcional definido pela fórmula 1.1, mas com domínio $C_{\tilde{x}_{0}}^{\tilde{x}_{1}}(M)$. Manteremos dois pontos $x_{0}, x_{1} \in M$ priviliegiados fazendo $I_{L}^{x_{0}, x_{1}}=I_{L}$

Lema 1.1.7. Sejam $M$ variedade Riemmaneana e $L: T M \rightarrow \mathbb{R}$ um Lagrangeano. Se $t_{1}, t_{2} \in[0,1]$ com $t_{1}<t_{2}$ e $\alpha:[0,1] \rightarrow M$, então se $\alpha$ é ponto crítico de $I_{L}$ temos que $\left.\alpha\right|_{\left[t_{1}, t_{2}\right]}$ é ponto crítico de $I_{L}^{\alpha\left(t_{1}\right), \alpha\left(t_{2}\right)}$.

Demonstração. Usando a fórmula 1.1 e a aditividade de integrais podemos ver que

$$
I_{L}(\alpha)=I_{L}^{\alpha(0), \alpha\left(t_{0}\right)}\left(\left.\alpha\right|_{\left[0, t_{0}\right]}\right)+I_{L}^{\alpha\left(t_{0}\right), \alpha\left(t_{1}\right)}\left(\left.\alpha\right|_{\left[t_{0}, t_{1}\right]}\right)+I_{L}^{\alpha\left(t_{1}\right), \alpha(1)}\left(\left.\alpha\right|_{\left[t_{1}, 1\right]}\right)
$$

Seja então $\tilde{h}:(-\epsilon, \epsilon) \times\left[t_{1}, t_{2}\right] \rightarrow M$ variação própria de $\left.\alpha\right|_{\left[t_{1}, t_{2}\right]}$ e defina $h:(-\epsilon, \epsilon) \times[0,1] \rightarrow M$ por

$$
h_{s}(t)= \begin{cases}\tilde{h}_{s}(t), & \text { se } t \in\left[t_{1}, t_{2}\right] \\ \alpha(t), & \text { caso contrário }\end{cases}
$$

$h$ é uma variação de $\alpha, \operatorname{logo} \frac{\mathrm{d}}{\mathrm{d} s} I_{L}\left(h_{s}\right)=0$. Como $I_{L}^{\alpha(0), \alpha\left(t_{0}\right)}\left(\left.h_{s}\right|_{\left[0, t_{0}\right]}\right)$ e $I_{L}^{\alpha\left(t_{1}\right), \alpha(1)}\left(\left.h_{s}\right|_{\left[t_{1}, 1\right]}\right)$ são constantes com relação a $s$, temos que

$$
\frac{\mathrm{d}}{\mathrm{d} s} I_{L}^{\alpha\left(t_{0}\right), \alpha\left(t_{1}\right)}\left(\tilde{h}_{s}\right)=0
$$

O lema 1.1.7 nos dá o seguinte corolário

Corolário 1.1.8. Sejam $M$ variedade Riemmaneana e $L: \mathrm{T} M \rightarrow \mathbb{R}$ um Lagrangeano. Então se $\alpha:[0,1] \rightarrow M$ é ponto crítico de $I_{L}, \alpha$ é solução de Euler-Lagrange em coordenadas segundo $L$.

Demonstração. Seja $\psi: U \subset \mathbb{R} \rightarrow V \subset M$ parametrização de $M$ tal que $V$ intercepta a imagem de $\alpha$. Considere $t_{1}, t_{2} \in[0,1]$ tais que $\alpha\left(\left[t_{1}, t_{2}\right]\right) \subset V$. Então a curva $\tilde{\alpha}=\left.\alpha\right|_{\left[t_{1}, t_{2}\right]}$ é ponto crítico de $I_{L}^{\alpha\left(t_{1}\right), \alpha\left(t_{2}\right)}$. Pelo lema 1.1.5, $\tilde{\alpha}$, e, portanto, $\alpha$, são de Euler-Lagrange em coordenadas segundo $L$ em todo $t_{0} \in\left[t_{1}, t_{2}\right]$. Como $\psi$ era carta arbitrária, isto prova o resultado.

O corolário 1.1.8 nos motiva a dar uma nova definição.

Definição 1.1.9. Sejam $M$ variedade Riemanneana e $L: T M \rightarrow \mathbb{R}$ um Lagrangeano. Dada uma curva $\alpha:[0,1] \rightarrow M$, diremos que $\alpha$ é solução de Euler-Lagrange segundo $L$ se $\alpha$ é ponto crítico de $I_{L}$.

Note que o corlário 1.1.8 nos garante se uma curva $\alpha$ é de Euler-Lagrange segundo um Lagrangeano $L$, então $\alpha$ é de Euler-Lagrange em coordenadas segundo $L$. Note também que, quando $M=\mathbb{R}^{n}$, o lema 1.1.3 nos garante que não há conflito de definições. 


\subsubsection{Equações de Newton}

Agora, com os resultados já provados, podemos ver que o formalismo introduzido realmente engloba ao menos parte da mecânica clássica de Newton. Se considerarmos um sistema com uma partícula de massa $m$, com posição entre os tempos 0 e 1 dada pela função $\alpha:[0,1] \rightarrow M$ e sujeita a uma força conservativa com potencial dado pela função $U: M \rightarrow \mathbb{R}$. Quando $M=\mathbb{R}^{n}$, a mecânica Newtoneana nos diria que a equação que rege o movimento dessa partícula é

$$
m \alpha^{\prime \prime}(t)=-\operatorname{grad} U(\alpha(t))
$$

Considere o Lagrangeano $L: \mathbb{R}^{n} \times \mathbb{R}^{n} \rightarrow \mathbb{R}$ dado por $L(q, \dot{q})=\frac{m}{2}\langle\dot{q}, \dot{q}\rangle-U(q)$. Afirmo que $\alpha$ é ponto crítico de $I_{L}$. De fato, o lema 1.1.3 nos diz que isso vale se, e só, se

$$
\begin{aligned}
\frac{\partial L}{\partial q_{i}}\left(\alpha(t), \alpha^{\prime}(t)\right) & =-\frac{\partial U}{\partial q_{i}}(\alpha(t)) \\
& =m \alpha_{i}^{\prime \prime}(t) \\
& =\frac{\mathrm{d}}{\mathrm{d} t} \frac{\partial L}{\partial \dot{q}_{i}}\left(\alpha(t), \alpha^{\prime}(t)\right)
\end{aligned}
$$

Logo encontrar a trajetória da partícula através do formalismo Newtoneano é equivalente a encontrar pontos críticos do funcional ação associado ao Lagrangeano acima.

Vamos agora considerar o caso em que $M$ é uma veriedade Riemanneana qualquer. Assumindo $U$ e $\alpha$ como acima, a equação de movimento de Newton assume a forma

$$
\nabla_{\alpha^{\prime}} \alpha^{\prime}=-\operatorname{grad} U
$$

onde $\nabla$ é a conexão Riemmaneana associada à métrica de $M$.

A proposição a seguir mostra que a noção de pontos críticos de um funcional ação continua capturando essa porção da mecânica.

Proposição 1.1.10. Sejam $M$ uma variedade Riemanniana, $\alpha:[0,1] \rightarrow M$ uma curva suave por partes, $U: M \rightarrow \mathbb{R}$ uma função suave e considere o lagrangeano $L\left(v_{p}\right)=\frac{1}{2}\left\langle v_{p}, v_{p}\right\rangle-U(p)$. Então $\alpha$ é solução de Euler-Lagrange segundo L se, e só se, $\alpha$ é suave e $\nabla_{\alpha^{\prime}} \alpha^{\prime}=-\operatorname{grad} U$.

Demonstração. Seja $h:[0,1] \times(-\epsilon, \epsilon) \rightarrow M$ uma variação própria de $\alpha$. Então, se as descontinuidades de $\alpha$ são $0=t_{0}=\ldots=t_{k+1}=1$, temos que

$$
\begin{aligned}
\frac{\mathrm{d}}{\mathrm{d} s} I_{L}\left(h_{s}\right) & =\frac{\mathrm{d}}{\mathrm{d} s} \int_{0}^{1} \frac{1}{2}\left\langle\frac{\mathrm{d} h_{s}}{\mathrm{~d} t}(t), \frac{\mathrm{d} h_{s}}{\mathrm{~d} t}(t)\right\rangle-U\left(h_{s}(t)\right) \mathrm{d} t \\
& =\sum_{i=0}^{k} \frac{\mathrm{d}}{\mathrm{d} s} \int_{t_{i}}^{t_{i+1}} \frac{1}{2}\left\langle\frac{\mathrm{d} h_{s}}{\mathrm{~d} t}(t), \frac{\mathrm{d} h_{s}}{\mathrm{~d} t}(t)\right\rangle-U\left(h_{s}(t)\right) \mathrm{d} t
\end{aligned}
$$

Analisando o termo do produto interno de cada integral do somatório, temos que

$$
\begin{aligned}
\frac{\mathrm{d}}{\mathrm{d} s} \int_{t_{i}}^{t_{i+1}} \frac{1}{2}\left\langle\frac{\mathrm{d} h_{s}}{\mathrm{~d} t}(t), \frac{\mathrm{d} h_{s}}{\mathrm{~d} t}(t)\right\rangle \mathrm{d} t & =\int_{t_{i}}^{t_{i+1}}\left\langle\nabla_{\frac{\mathrm{d} h s}{\mathrm{~d} s}} \frac{\mathrm{d} h_{s}}{\mathrm{~d} t}(t), \frac{\mathrm{d} h_{s}}{\mathrm{~d} t}(t)\right\rangle \mathrm{d} t \\
& =\int_{t_{i}}^{t_{i+1}}\left\langle\nabla_{\frac{\mathrm{d} h_{s}}{\mathrm{~d} t}} \frac{\mathrm{d} h_{s}}{\mathrm{~d} s}(t), \frac{\mathrm{d} h_{s}}{\mathrm{~d} t}(t)\right\rangle \mathrm{d} t
\end{aligned}
$$

onde a primeira igualdade se dá devido ao fato de a integral em $t$ e a derivada em $s$ comutarem e 
a segunda, graças à simetria da conexão. Aplicando a regra do produto à última integral, temos

$$
\begin{aligned}
\int_{t_{i}}^{t_{i+1}}\left\langle\nabla_{\frac{\mathrm{d} h_{s}}{\mathrm{~d} t}} \frac{\mathrm{d} h_{s}}{\mathrm{~d} s}(t), \frac{\mathrm{d} h_{s}}{\mathrm{~d} t}(t)\right\rangle \mathrm{d} t & =\int_{t_{i}}^{t_{i+1}} \frac{\mathrm{d}}{\mathrm{d} t}\left\langle\frac{\mathrm{d} h_{s}}{\mathrm{~d} s}(t), \frac{\mathrm{d} h_{s}}{\mathrm{~d} t}(t)\right\rangle \mathrm{d} t-\int_{t_{i}}^{t_{i+1}}\left\langle\frac{\mathrm{d} h_{s}}{\mathrm{~d} s}(t), \nabla_{\frac{\mathrm{d} h_{s}}{\mathrm{~d} t}} \frac{\mathrm{d} h_{s}}{\mathrm{~d} t}(t)\right\rangle \mathrm{d} t \\
& =\left.\left\langle\frac{\mathrm{d} h_{s}}{\mathrm{~d} s}(t), \frac{\mathrm{d} h_{s}}{\mathrm{~d} t}(t)\right\rangle\right|_{t=t_{i}} ^{t=t_{i+1}}-\int_{t_{i}}^{t_{i+1}}\left\langle\frac{\mathrm{d} h_{s}}{\mathrm{~d} s}(t), \nabla_{\frac{\mathrm{d} h_{s}}{\mathrm{~d} t}} \frac{\mathrm{d} h_{s}}{\mathrm{~d} t}(t)\right\rangle \mathrm{d} t
\end{aligned}
$$

Logo, cada parcela do somatório pode ser escrita

$$
\frac{\mathrm{d}}{\mathrm{d} s} \int_{t_{i}}^{t_{i+1}} \frac{1}{2}\left\langle\frac{\mathrm{d} h_{s}}{\mathrm{~d} t}(t), \frac{\mathrm{d} h_{s}}{\mathrm{~d} t}(t)\right\rangle-U\left(h_{s}(t)\right) \mathrm{d} t=\left.\left\langle\frac{\mathrm{d} h_{s}}{\mathrm{~d} s}(t), \frac{\mathrm{d} h_{s}}{\mathrm{~d} t}(t)\right\rangle\right|_{t=t_{i}} ^{t=t_{i+1}}-\int_{t_{i}}^{t_{i+1}}\left\langle\frac{\mathrm{d} h_{s}}{\mathrm{~d} s}(t), \nabla_{\frac{\mathrm{d} h_{s}}{\mathrm{~d} t}} \frac{\mathrm{d} h_{s}}{\mathrm{~d} t}(t)+\operatorname{grad} U(t)\right\rangle \mathrm{d} t
$$

Somando todas as parcelas, fazendo $s=0$ e lembrando que $h$ é própria, obtemos

$$
\left.\frac{\mathrm{d}}{\mathrm{d} s} I_{L}\left(h_{s}\right)\right|_{s=0}=\sum_{i=1}^{k}\left\langle\left.\frac{\mathrm{d} h_{s}}{\mathrm{~d} s}\left(t_{i}\right)\right|_{s=0}, \alpha^{\prime}\left(t_{i}^{+}\right)-\alpha^{\prime}\left(t_{i}^{-}\right)\right\rangle-\int_{0}^{1}\left\langle\left.\frac{\mathrm{d} h_{s}}{\mathrm{~d} s}(t)\right|_{s=0}, \nabla_{\alpha^{\prime}} \alpha^{\prime}(t)+\operatorname{grad} U(t)\right\rangle \mathrm{d} t
$$

onde $\alpha^{\prime}\left(t_{i}^{+}\right)=\lim _{t \rightarrow t_{i}^{+}}$e $\alpha^{\prime}\left(t_{i}^{-}\right)=\lim _{t \rightarrow t_{i}^{-}}$.

Suponha que $\alpha$ é solução de Euler-Lagrange segundo $L$. Então, podemos escolher $h$ tal que para cada $i \in\{1, \ldots, k+1\}, h_{s}\left(t_{i}\right)$ é função constante de $s$. Logo o primeiro termo da direita é nulo e, usando o lema 1.1.2 para cada intervalo $\left[t_{i}, t_{i+1}\right]$, concluimos que $\nabla_{\alpha^{\prime}} \alpha^{\prime}=-\operatorname{grad} U$. Mas isso nos deixa apenas com o primeiro termo, e podendo variar os valores de $h_{s}\left(t_{i}\right)$ livremente, concluimos que $\alpha^{\prime}\left(t_{i}^{+}\right)-\alpha^{\prime}\left(t_{i}^{-}\right)=0$ para $0 \leq i \leq n, \log \alpha$ é suave.

Por outro lado, se $\alpha$ é suave e $\nabla_{\alpha^{\prime}} \alpha^{\prime}=-\operatorname{grad} U$, a equação 1.5 nos garante que $\alpha$ é solução de Euler-Lagrange segundo $L$.

Note que, no lema acima, se considerarmos $U \equiv 0$, temos o seguinte corolário

Corolário 1.1.11. Se $M$ é uma variedade Riemmaneana com métrica g, então uma curva $\alpha$ é geodésica em $M$ se, e só se, $\alpha$ é solução de Euler-Lagrange para o Lagrangeano $L(v)=g(v, v)$, ou seja, $\alpha$ é geodésica para a métrica $g$

Vamos agora considerar um sitema físico conservativo, cuja força tem potencial dado por uma função $U: M \rightarrow \mathbb{R}$ limitada, ou seja, tal que existe $c \in \mathbb{R}$ tal que $U(p)<c$ para todo $p \in M$. A proposição a seguir nos diz que podemos encontrar uma métrica $g_{J}$ para $M$, chamada métrica de Jacobi, tal que as soluções físicas do sistema dado correspondem a geodésicas de $g_{J}$. A prova desse teorema requer alguns resultados da formulação Hamiltoneana e, portanto, deixaremos sua prova para a seção 1.2.3.

Teorema 1.1.12. Considere $M$ variedade Riemanneana dotada de métrica $g$ e $U: M \rightarrow \mathbb{R}$ função suave com $U(q)<c, \forall q \in M$, para algum $c \in \mathbb{R}$. Se $L: T M \rightarrow \mathbb{R}$ é um Lagrangeano dado por $L\left(v_{p}\right)=\left\langle v_{p}, v_{p}\right\rangle-U(p)$ e $g_{J}$ é uma métrica em $M$ tal que $\left(g_{J}\right)_{q}=(c-U(q)) g_{q}$, então uma curva $\alpha:[0,1] \rightarrow M$ é de Euler-Lagrange segundo $L$ se, e só se, $\alpha$ é geodésica de $g_{J}$, a menos de reparametrização.

\subsubsection{Transformação de Legendre e Energia}

Aqui introduziremos algumas ferramentas construidas com base no Lagrangeano, mas que serão o fundamento do formalismo Hamiltoneano. Essas ferramentas são a transformação de Legendre e a energia. A transformação de Legendre é um isomorfismo induzido pelo Lagrangeano que identifica o fibrado tangente e o fibrado cotangente de uma variedade. O fibrado cotangente é o protótipo de uma variedade simplética, como veremos à frente e usaremos essa estrutura a nosso favor quando formos desenvolver o formalismo Hamiltoneano. A energia é um funcional criado a partir da transformação de Legendre e do Lagrangeano, que será central no formalismo Hamiltoneano. Na verdade, veremos 
que o formalismo Hamiltoneano pode ser resumido a encontrar soluções do fluxo do funcional energia.

Dado um Lagrangeano $\mathrm{T} M \rightarrow \mathbb{R}$, definimos a transformação de Legendre $T_{L}: \mathrm{T} M \rightarrow \mathrm{T}^{*} M$ da seguinte forma

$$
T_{L}(v) u=\left.\frac{\mathrm{d}}{\mathrm{d} t} L(v+t u)\right|_{t=0}
$$

para todos $v, u \in \mathrm{T}_{x} M$, para cada $x \in M$.

O lema a seguir descreve a transformação de Legendre em coordenadas. Lembre-se que, se $\psi: U \subset \mathbb{R}^{n} \rightarrow V \subset M$ é uma carta de $M$, então a função d $\psi: \mathrm{T} U \subset \mathrm{TR}^{n} \rightarrow \mathrm{T} V \subset \mathrm{T} M$, é um carta para $\mathrm{T} M$ e $\psi^{*}: \mathrm{T}^{*} V \subset \mathrm{T}^{*} M \rightarrow \mathrm{T}^{*} V \subset \mathrm{T}^{*} \mathbb{R}^{n}$ é um sistema de coordenadas de $\mathrm{T}^{*} M$.

Lema 1.1.13. Seja $\psi: U \subset \mathbb{R}^{2 n} \rightarrow V \subset \mathrm{T} M$ sistema de coordenadas para $\mathrm{T} M$, e $L: \mathrm{T} M \rightarrow \mathbb{R}$. Então

$$
\begin{aligned}
\psi^{*} \circ T_{L} \circ \mathrm{d} \psi(q, \dot{q}) & =T_{L_{\psi}}(q, \dot{q}) \\
& =\left(q, \frac{\partial L_{\psi}}{\partial \dot{q}}(q, \dot{q})\right)
\end{aligned}
$$

onde $L_{\psi}=L \circ \mathrm{d} \psi$. Ou seja, o diagram abaixo comuta

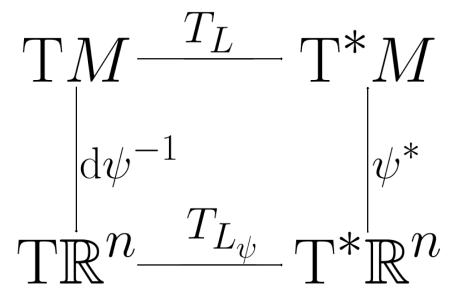

Figura 1.1: Diagrama transformação de Legendre e coordenadas

Demonstração. Note primeiro que $\tilde{T}_{L}(q, \dot{q}) \in T^{*} \mathbb{R}^{n}$ e que, se $v \in \mathrm{T}_{q} \mathbb{R}^{n} \approx \mathbb{R}^{n}$

$$
\begin{aligned}
\psi^{*} \circ T_{L} \circ \mathrm{d} \psi(q, \dot{q})(q, v) & =T_{L}(\mathrm{~d} \psi \dot{q})(\mathrm{d} \psi v) \\
& =\left.\frac{\mathrm{d}}{\mathrm{d} t} L\left(\mathrm{~d} \psi_{q} \dot{q}+t \mathrm{~d} \psi_{q} v\right)\right|_{t=0} \\
& =\left.\frac{\mathrm{d}}{\mathrm{d} t}(L \circ \mathrm{d} \psi)(q, \dot{q}+t v)\right|_{t=0} \\
& =T_{L_{\psi}}(q, \dot{q})(q, v)
\end{aligned}
$$

O que nos dá a primeira igualdade. Para provar a segunda, observe apenas que

$$
\begin{aligned}
T_{L_{\psi}}(q, \dot{q})(q, v) & =\left.\frac{\mathrm{d}}{\mathrm{d} t}\left(L_{\psi}\right)(q, \dot{q}+t v)\right|_{t=0} \\
& =\left(q, \frac{\partial L_{\psi}}{\partial \dot{q}}(q, \dot{q})(v)\right)
\end{aligned}
$$

Para dar uma motivação à existência da transformação de Legendre, vamos analisá-la no caso $M=\mathbb{R}^{n}$ com o Lagrangeano usado na mecânica de Newton, a lembrar $L(q, \dot{q})=\frac{m}{2}\langle\dot{q}, \dot{q}\rangle-U(q)$. Neste caso, como a identidade é uma carta, o lema acima nos diz que

$$
\begin{aligned}
T_{L}(q, \dot{q})(q, v) & =\left.\frac{m}{2} \frac{\mathrm{d}}{\mathrm{d} t}(\langle\dot{q}+t v, \dot{q}+t v\rangle-U(q))\right|_{t=0} \\
& =\langle m \dot{q}, v\rangle
\end{aligned}
$$


Ou seja, temos $T_{L}(q, \dot{q})=(q,\langle m \dot{q}, \cdot\rangle)$. Mas, lembrando que $\dot{q}$ representa a componente das velocidades do espaço de fase, temos que $m \dot{q}$ é o momento linear. Logo, no caso Newtoneano a transformação de Legendre é uma mudança de coordenadas do par espaço-velocidade para espaçomomento.

Em posse da transformação de Legendre, podemos definir a energia associada a $L$.

Definição 1.1.14. Seja $M$ variedade diferenciável e $L: \mathrm{T} M \rightarrow \mathbb{R}$ é um Lagrangeano, definimos a energia associada a $L$ como o funcional $E_{L}: \mathrm{T} M \rightarrow \mathbb{R}$ dado por

$$
E(v)=T_{L}(v) v-L(v)
$$

Pelo lema 1.1.13, temos que, se $\psi$ é uma parametrização de $M$, então

$$
\begin{aligned}
E_{L_{\psi}}(q, \dot{q}) & =\frac{\partial L_{\psi}}{\partial \dot{q}}(q, \dot{q}) \dot{q}-L_{\psi}(q, \dot{q}) \\
& =\left\langle\frac{\partial L_{\psi}}{\partial \dot{q}}(q, \dot{q}), \dot{q}\right\rangle-L_{\psi}(q, \dot{q})
\end{aligned}
$$

sendo que na última igualdade usamos a identificação do $\mathbb{R}^{n}$ com o seu dual via produto interno.

Note, agora, que, se $\alpha$ é solução de Euler-Lagrange segundo $L$ temos

$$
\frac{\mathrm{d}}{\mathrm{d} t}\left\langle\frac{\partial L_{\psi}}{\partial \dot{q}}\left(\alpha(t), \alpha^{\prime}(t)\right), \alpha^{\prime}(t)\right\rangle=\left\langle\frac{\mathrm{d}}{\mathrm{d} t} \frac{\partial L_{\psi}}{\partial \dot{q}}\left(\alpha(t), \alpha^{\prime}(t)\right), \alpha^{\prime}(t)\right\rangle+\left\langle\frac{\partial L_{\psi}}{\partial \dot{q}}\left(\alpha(t), \alpha^{\prime}(t)\right), \alpha^{\prime \prime}(t)\right\rangle
$$

e também que

$$
\frac{\mathrm{d}}{\mathrm{d} t} L_{\psi}\left(\alpha(t), \alpha^{\prime}(t)\right)=\left\langle\frac{\partial L_{\psi}}{\partial q}\left(\alpha(t), \alpha^{\prime}(t)\right), \alpha^{\prime}(t)\right\rangle+\left\langle\frac{\partial L_{\psi}}{\partial \dot{q}}\left(\alpha(t), \alpha^{\prime}(t)\right), \alpha^{\prime \prime}(t)\right\rangle
$$

Assim obtemos que $\frac{\mathrm{d}}{\mathrm{d} t} E_{L_{\psi}}\left(\alpha(t), \alpha^{\prime}(t)\right)=0$. Logo a energia é constante ao longo das soluções de Euler-Lagrange para seu Lagrangeano associado. Assim, a cada solução de Euler-Lagrange $\alpha$ para um Lagrangeano $L$ podemos associar uma energia de valor $E_{L}(\alpha(0))$. Podemos também associar uma energia a uma geodésica para uma métrica $g$, lembrando que, pelo corolário 1.1.11, ela é solução de Euler-Lagrange para $L(v)=g(v, v)$

Considerando novamente o caso físico em que $M=\mathbb{R}^{n} \operatorname{com} L(q, \dot{q})=\frac{m}{2}\langle\dot{q}, \dot{q}\rangle-U(q)$, temos que

$$
\begin{aligned}
E_{L_{\psi}}(q, \dot{q}) & =\langle m \dot{q}, \dot{q}\rangle-\frac{m}{2}\langle\dot{q}, \dot{q}\rangle+U(q) \\
& =m\langle\dot{q}, \dot{q}\rangle+U(q)
\end{aligned}
$$

e, portanto, $E_{L}$ é soma das energias cinética e potencial, que é a energia total de um problema apenas com forças conservativas.

\subsubsection{Teorema de Noether}

Nesta seção, provaremos um importante resultado da formulação Lagrangeana. O teorema de Noether afirma que, em um sistema descrito por um Lagrangeano seguindo o princípio da ação mínima, a toda simetria do Lagrangeano está associada uma lei de conservação. Apresentaremos agora o enunciado formal do teorema.

Teorema 1.1.15 (Noether). Sejam $M$ uma variedade Riemanneana, $g: \mathbb{R} \times M \rightarrow M$ um grupo de difeomorfismos a um parâmetro e $L: \mathrm{T} M \rightarrow \mathbb{R}$ um Lagrangeano invariante por $g_{s}$. Se $\mathcal{I}: \mathrm{T} M \rightarrow \mathbb{R}$ é definido por

$$
\mathcal{I}_{L}(v)=T_{L}(v)\left(\left.\frac{\mathrm{d}}{\mathrm{d} s} g_{s}(p)\right|_{s=0}\right)
$$

onde $p=\pi(v)$, então para toda curva $\alpha$ solução de Euler-Lagrange para L, temos que $\mathcal{I}\left(\alpha^{\prime}\right)$ é constante. 
Demonstração. Seja $\psi: U \subset M \rightarrow V \subset \mathbb{R}^{n}$ carta de $M$ e defina $\tilde{\alpha}=\psi \circ \alpha, \tilde{g}_{s}=\psi \circ g_{s} \circ \psi^{-1} \mathrm{e}$ $L_{\psi}=L \circ \mathrm{d} \psi$. Temos então que

$$
\begin{aligned}
\mathcal{I}_{L}\left(\alpha^{\prime}\right) & =T_{L}\left(\alpha^{\prime}\right)\left(\left.\frac{\mathrm{d}}{\mathrm{d} s} g_{s}(\alpha)\right|_{s=0}\right) \\
& =T_{L_{\psi}}\left(\tilde{\alpha}, \tilde{\alpha}^{\prime}\right) \circ \mathrm{d} \psi\left(\left.\mathrm{d} \psi^{-1} \circ \frac{\mathrm{d}}{\mathrm{d} s}\left(\tilde{g}_{s}\right) \circ \psi\left(\psi^{-1}(\alpha)\right)\right|_{s=0}\right) \\
& =T_{L_{\psi}}\left(\tilde{\alpha}, \tilde{\alpha}^{\prime}\right)\left(\left.\frac{\mathrm{d}}{\mathrm{d} s} \tilde{g}_{s}(\alpha)\right|_{s=0}\right) \\
& =\left\langle\frac{\partial L_{\psi}}{\partial \dot{q}}\left(\tilde{\alpha}, \tilde{\alpha}^{\prime}\right),\left.\frac{\mathrm{d}}{\mathrm{d} s} \tilde{g}_{s}(\alpha)\right|_{s=0}\right\rangle
\end{aligned}
$$

Assim, precisamos apenas provar que $\left\langle\frac{\partial L_{\psi}}{\partial \dot{q}}\left(\tilde{\alpha}, \tilde{\alpha}^{\prime}\right),\left.\frac{\mathrm{d}}{\mathrm{d} s} \tilde{g}_{s}(\alpha)\right|_{s=0}\right\rangle$ é constante. Mas observe que

$$
\begin{aligned}
\frac{\mathrm{d}}{\mathrm{d} t}\left\langle\frac{\partial L_{\psi}}{\partial \dot{q}}\left(\tilde{\alpha}(t), \tilde{\alpha}^{\prime}(t)\right),\left.\frac{\mathrm{d}}{\mathrm{d} s} \tilde{g}_{s}(\alpha(t))\right|_{s=0}\right\rangle= & \left\langle\frac{\mathrm{d}}{\mathrm{d} t} \frac{\partial L_{\psi}}{\partial \dot{q}}\left(\tilde{\alpha}(t), \tilde{\alpha}^{\prime}(t)\right),\left.\frac{\mathrm{d}}{\mathrm{d} s} \tilde{g}_{s}(\alpha(t))\right|_{s=0}\right\rangle \\
& +\left\langle\frac{\partial L_{\psi}}{\partial q}\left(\tilde{\alpha}(t), \tilde{\alpha}^{\prime}(t)\right),\left.\frac{\mathrm{d}}{\mathrm{d} t} \frac{\mathrm{d}}{\mathrm{d} s} \tilde{g}_{s}(\alpha(t))\right|_{s=0}\right\rangle \\
= & \left\langle\frac{\partial L_{\psi}}{\partial \dot{q}}\left(\tilde{\alpha}(t), \tilde{\alpha}^{\prime}(t)\right),\left.\frac{\mathrm{d}}{\mathrm{d} s} \tilde{g}_{s}(\alpha(t))\right|_{s=0}\right\rangle \\
& +\left\langle\frac{\partial L_{\psi}}{\partial \dot{q}}\left(\tilde{\alpha}(t), \tilde{\alpha}^{\prime}(t)\right),\left.\frac{\mathrm{d}}{\mathrm{d} s} \frac{\mathrm{d}}{\mathrm{d} t} \tilde{g}_{s}(\alpha(t))\right|_{s=0}\right\rangle \\
= & \frac{\partial}{\partial s} L_{\psi}\left(\tilde{g}_{s} \circ \alpha(t), \frac{\mathrm{d}}{\mathrm{d} t} \tilde{g}_{s} \circ \alpha(t)\right) \\
= & \frac{\partial}{\partial s} L_{\psi}\left(\alpha(t), \alpha^{\prime}(t)\right) \\
= & 0
\end{aligned}
$$

Para ilustrar a utilidade do teorema de Noether, o exemplo a seguir irá usá-lo para deduzir a conservação do momento angular em sistemas conservativos com simetria radial.

Exemplo 1.1.16. Considere $L: \mathbb{T R}^{3} \approx \mathbb{R}^{6} \rightarrow \mathbb{R}$ o Lagrangeano Newtoneano $L(q, \dot{q})=\frac{m}{2}\langle\dot{q}, \dot{q}\rangle-U(q)$ tal que $U$ seja invariante por rotações. Dado $\eta=\left(\eta_{1}, \eta_{2}, \eta_{3}\right) \in \mathbb{R}^{3}$, defina a matriz

$$
A_{\eta}=\left[\begin{array}{ccc}
0 & -\eta_{3} & \eta_{2} \\
\eta_{3} & 0 & -\eta_{1} \\
-\eta_{2} & \eta_{1} & 0
\end{array}\right] \in \mathfrak{s o}(3)
$$

Usaremos aqui o fato de que, dado $v \in \mathbb{R}^{3}, A_{\eta} v=\eta \times v$, onde $\times$ é o produto vetorial. Esse resultado pode ser verificado apenas calculando ambos os termos e não será provado.

Considere $g_{s}^{\eta}=\exp (s \eta)$ grupo a 1 parâmetro de matrizes de $\mathrm{SO}(3)$. Como $U$ é invariante por rotações e a ação de $\mathrm{SO}(3)$ em $\mathbb{R}^{3}$ preserva a norma, temos que $L$ é invariante por $g_{s}$. Assim, o teorema de Noether nos garante que

$$
\begin{aligned}
\mathcal{I}_{L}(q, \dot{q}) & =\left\langle\frac{\partial L}{\partial \dot{q}}(q, \dot{q}),\left.\frac{\mathrm{d}}{\mathrm{d} s} \tilde{g}_{s}(\alpha)\right|_{s=0}\right\rangle \\
& =\left\langle m \dot{q}, A_{\eta} q\right\rangle \\
& =\langle m \dot{q}, \eta \times q\rangle \\
& =\langle\eta \times q, m \dot{q}\rangle \\
& =\langle q \times(m \dot{q}), \eta\rangle
\end{aligned}
$$

é constante ao longo das soluções de Euler-Lagrange de $L$. Como $\eta$ foi escolhido arbitrariamente, 
temos que $q \times(m \dot{q})$ o momento angular é constante.

\subsection{Formulação Hamiltoneana}

A formulação Hamiltoneana usa geometria simplética para encontrar curvas que preservem alguma grandeza, no caso da física, a energia. Antes de introduzir esse formalismo faremos uma revisão de conceitos básicos de geometria simplética.

\subsubsection{Rudimentos de Geometria Simplética}

Definição 1.2.1. Se $V$ é um espaço vetorial, uma estrutura simplética linear em $V$ é um 2-tensor alternado $\omega \in A^{2}(V)$ não-degenerado. Da mesma forma, se $M$ é uma variedade diferenciável, uma forma simplética em $M$ é uma 2-forma $\omega \in \Omega^{2}(M)$ fechada e não-degenerada.

Se $V$ é um espaço vetorial e $\omega$ é uma estrutura simplética linear em $V$, então o par $(V, \omega)$ é dito um espaço vetorial simplético ou apenas espaço simplético. Analogamente, se $M$ é uma variedade diferenciável e $\omega$ é uma forma simplética em $M$, então o par $(M, \omega)$ é dito uma variedade simplética. Note que todo espaço vetorial simplético pode ser considerado uma variedade simplética, onde o tensor alternado é visto como uma forma constante, que é automaticamente fechada.

Frequentemente denotaremos uma variedade simplética apenas pela variedade $M$, omitindo a forma simplética. Nesses casos $\omega$ denotará suas forma simplética, a menos quando especificado o contrário.

Muitas vezes iremos querer saber se dois espaços simpléticos são equivalentes. A definição a seguir nos dá uma forma de saber se duas variedades simpléticas são intercambiáveis.

Definição 1.2.2. Sejam $\left(M_{1}, \omega_{1}\right)$ e $\left(M_{2}, \omega_{2}\right)$ variedades simpléticas. Se $f: M_{1} \rightarrow M_{2}$ é um difeomorfismo tal que $\omega_{1}=f^{*} \omega_{2}$, ele é dito um simplectomorfismo. $\left(M_{1}, \omega_{1}\right)$ e $\left(M_{2}, \omega_{2}\right)$ são ditas simplectomorfas se existir um simplectomorfismo entre elas.

\section{Exemplos}

Exemplo 1.2.3 (Espaço Euclidiano). O primeiro exemplo de espaço simplético é o espaço $\mathbb{R}^{2 n}$. Aqui consideraremos $\mathbb{R}^{2 n}=\mathbb{R}^{n} \times \mathbb{R}^{n}$ e escreveremos $v=\left(v_{1}, v_{2}\right) \in \mathbb{R}^{2 n}$, com $v_{1}, v_{2} \in \mathbb{R}^{n}$. Defina $\omega: \mathbb{R}^{2 n} \times \mathbb{R}^{2 n} \times \mathbb{R}$ tal que

$$
\omega\left(\left(u_{1}, u_{2}\right),\left(v_{1}, v_{2}\right)\right)=\left\langle u_{1}, v_{2}\right\rangle-\left\langle v_{1}, u_{2}\right\rangle
$$

Vamos mostrar que $\omega$ é simplética. Que $\omega$ é bilinear e anti-simétrica é óbvio da definição. Para ver que $\omega$ é não-degenerada, seja $\left(u_{1}, u_{2}\right) \in \mathbb{R}^{2 n}$ tal que $\omega\left(\left(u_{1}, u_{2}\right),(\cdot)\right)=0$. Logo $\omega\left(\left(u_{1}, u_{2}\right),\left(0, v_{2}\right)\right)=$ $\left\langle u_{1}, v_{2}\right\rangle=0$, para todo $v_{2} \in \mathbb{R}^{n}$, o que implica $u_{1}=0$. Por um argmuento similar temos também $u_{2}=0$.

$\omega$ é chamada a forma simplética canônica de $\mathbb{R}^{2 n}$.

Será interessante mais adiante ter uma descrição de $\omega$ em coordenadas. Considere, então, $f_{1}, \ldots, f_{n}$ a base canônica de $\mathbb{R}^{n}$ e defina $e_{i}=\left(f_{i}, 0\right) \in \mathbb{R}^{2 n}$ e $\tilde{e}_{i}=\left(0, f_{i}\right) \in \mathbb{R}^{2 n}$. Se definir$\operatorname{mos} \mathrm{d} x_{i}: \mathbb{R}^{2 n} \rightarrow \mathbb{R}$ como a projeção sobre o espaço gerado pelos $e_{i}$ e $\mathrm{d} y_{i}: \mathbb{R}^{2 n} \rightarrow \mathbb{R}$ o equivalente para $\tilde{e}_{i}$, temos que $\mathrm{d} x_{i}$ e $\mathrm{d} y_{i}$ são 1 -formas e que

$$
\omega=\sum_{i=1}^{n} \mathrm{~d} x_{i} \wedge \mathrm{d} y_{i}
$$

Da mesma forma temos que o espaço $\mathbb{C}^{n}$ também é simplético. Já que os espaços são isomorfos, podemos usar a mesma forma simplética, mas no caso $\mathbb{C}^{n}$ usaremos um múltiplo e o escreveremos em termos das 1 -formas $\mathrm{d} z_{i}=\mathrm{d} x_{i}+\mathrm{d} y_{i}$ e $\mathrm{d} \bar{z}_{i}=\mathrm{d} x_{i}-\mathrm{d} y_{i}$. A nova forma simplética então é dada 
por

$$
-2 \omega=\sum_{i=1}^{n} \mathrm{~d} z_{i} \wedge \mathrm{d} \bar{z}_{i}
$$

A igualdade vale pois $\mathrm{d} z_{i} \wedge \mathrm{d} \bar{z}_{i}=-2 \mathrm{~d} x_{i} \wedge \mathrm{d} y_{i}$

Exemplo 1.2.4 (Fibrado Cotangente). O exemplo de variedade simplética que mais usaremos, é o fibrado cotangente. Dada uma variedade $M$, seu fibrado cotangente definido por

$$
\mathrm{T}^{*} M=\bigcup_{p \in M} \mathrm{~T}_{p} M^{*}
$$

Esse fibrado tem uma forma simplética natural, definida da seguinte maneira:

Vamos primeiro definir $\lambda \in \Lambda \mathrm{T}^{*} M$ a forma estrutural ou forma tautoógica de $\mathrm{T}^{*} M$. Dados $\pi: \mathrm{T}^{*} M \rightarrow M$ a projeção canônica do fibrado cotangente, $\alpha \in \mathrm{T}^{*} M$ e $v \in \mathrm{T}_{\alpha} \mathrm{T}^{*} M$, então, se $x=\pi(\alpha)$,

$$
\lambda_{\alpha}(v)=\alpha_{x}\left(\mathrm{~d} \pi_{x}(v)\right)
$$

Definimos a forma simplética canônica de $\mathrm{T}^{*} M$ como $\omega=-\mathrm{d} \lambda$. Para checar que $\omega$ é realmente simplética, precisamos mostrar que ela é fechada e não-degenerada. Da definição já segue que a forma é fechada, resta apenas verificar que é não-degenerada.

Para isso, vamos analisá-la em coordenadas. Seja $\psi: U \subset \mathbb{R}^{2 n} \rightarrow V \subset \mathrm{T}^{*} M$ paramterização de $\mathrm{T}^{*} M$. Podemos escrever $\psi^{-1}=\left(q_{1}, \ldots, q_{n}, p_{n}, \ldots, p_{n}\right)$. Assim, o funcional $\alpha \in \mathrm{T}^{*} M$ pode ser escrito

$$
\alpha=\sum_{i=1}^{n} p_{i}(\alpha) \mathrm{d} q_{i}(\alpha)
$$

$\mathrm{O}$ vetor $v \in \mathrm{T}_{\omega} \mathrm{T}^{*} M$ pode ser escrito

$$
v=\sum_{i=1}^{n} a_{i} \frac{\partial}{\partial q_{i}}(\omega)+\sum_{i=1}^{n} b_{i} \frac{\partial}{\partial p_{i}}(\omega)
$$

para algum $\left(a_{1}, \ldots, a_{n}, b_{1}, \ldots, b_{n}\right) \in \mathbb{R}^{2 n}$. Finalmente, a forma estrutural $\lambda$ pode ser escrita

$$
\lambda=\sum_{i=1}^{n} A_{i} \mathrm{~d} q_{i}+\sum_{i=1}^{n} B_{i} \mathrm{~d} p_{i}
$$

com $A_{1}, \ldots, A_{n}, B_{1}, \ldots, B_{n}$ funções de $\mathrm{T}^{*} M$ para $\mathbb{R}$. Assim, podemos reescrever a fórmula (1.7) obtendo

$$
\sum_{i=1}^{n}\left(a_{i} A_{i}+b_{i} B_{i}\right)=\sum_{i=1}^{n} a_{i} p_{i}
$$

para todo $\left(a_{1}, \ldots, a_{n}, b_{1}, \ldots, b_{n}\right) \in \mathbb{R}^{2 n}$. Logo $\lambda=\sum_{i=1}^{n} p_{i} \mathrm{~d} q_{i}$. A fórmula em coordenadas da derivada exterior nos dá

$$
\begin{aligned}
\omega & =-\mathrm{d} \lambda \\
& =-\sum_{i=1}^{n} \mathrm{~d} p_{i} \wedge \mathrm{d} q_{i} \\
& =\sum_{i=1}^{n} \mathrm{~d} q_{i} \wedge \mathrm{d} p_{i} \\
& =\sum_{i=1}^{n} \mathrm{~d} q_{i} \otimes \mathrm{d} p_{i}-\sum_{i=1}^{n} \mathrm{~d} p_{i} \otimes \mathrm{d} q_{i}
\end{aligned}
$$


Assim, se $\alpha, \beta \in \mathrm{T}^{*} M$, escritos nas coordenadas $\left(q_{1}, \ldots, q_{n}, p_{n}, \ldots, p_{n}\right)$ como $\left(\alpha_{1}, \alpha_{2}\right),\left(\beta_{1}, \beta_{2}\right)$, $\operatorname{com} \alpha_{1}, \alpha_{2}, \beta_{1}, \beta_{2} \in \mathbb{R}^{n}$, temos em notação matricial

$$
\omega(\alpha, \beta)=\left[\begin{array}{ll}
\alpha_{1} & \alpha_{2}
\end{array}\right]\left[\begin{array}{cc}
0 & \operatorname{Id} \\
-\operatorname{Id} & 0
\end{array}\right]\left[\begin{array}{l}
\beta_{1} \\
\beta_{2}
\end{array}\right]=\left\langle\alpha_{1}, \beta_{2}\right\rangle-\left\langle\alpha_{2}, \beta_{1}\right\rangle
$$

Portanto, se $\alpha \in \mathrm{T}^{*} M$ é tal que $\omega(\alpha, \beta)=0$ para todo $\beta \in \mathrm{T}^{*} M$, temos, escolhendo $\beta$ da forma $\left(\beta_{1}, 0\right)$, que $\alpha_{2}=0$ e com $\left(0, \beta_{2}\right)$ temos que $\alpha_{1}=0$, provandop a não-degenrescência de $\omega$.

Observação 1.2.5. Note que não foi feita nenhuma exigência especial da carta $\psi$. Logo, no caso em que $M=\mathbb{R}^{n}$, temos que $\mathrm{T}^{*} M \approx \mathbb{R}^{2 n}$ e $\operatorname{Id}_{\mathbb{R}^{2 n}}$ é uma carta de $\mathrm{T}^{*} M$. Dessa forma, $\omega$ é dada pela fórmula 1.8, que coincide com a forma simplética canônica de $\mathbb{R}^{2 n}$.

Em certos casos será interessante considerar produtos cartesianos de variedades simpléticas. A definição abaixo nos dá uma maneira de construir uma forma simplética para esse produto.

Definição 1.2.6. Sejam $\left(M_{1}, \omega_{1}\right)$ e $\left(M_{2}, \omega_{2}\right)$ variedades simpléticas. A variedade simplética produto de $M_{1}$ e $M_{2}$ é o par $\left(M_{1} \times M_{2}, \omega\right)$, onde, se $v_{1}, w_{1} \in M_{1}$ e $v_{2}, w_{2} \in M_{2}$

$$
\omega\left(\left(v_{1}, v_{2}\right),\left(w_{1}, w_{2}\right)\right)=\omega_{1}\left(v_{1}, w_{1}\right)+\omega_{2}\left(v_{2}, w_{2}\right)
$$

$\omega$ é chamada a forma simplética produto de $\omega_{1} e \omega_{2}$.

\section{Teorema de Darboux}

Nesta seção provaremos o teorema de Darboux, que afirma que localmente, toda variedade simplética simplética é simplectomorfa ao $\mathbb{R}^{2 n}$ dotado de sua forma simplética canônica. Essa seção é baseada em [6], palestras 34 e 35 .

Provaremos esse teorema primeiro para o caso particular de espaços vetoriais simpléticos. Começaremos com o seguinte lema

Lema 1.2.7. Seja $V$ espaço vetorial e $\omega \in A^{2}(V)$. Então existe $\left\{f_{1}, \ldots, f_{2 k}, f_{2 k+1}, \ldots, f_{n}\right\}$ base de $V^{*}$ tal que

$$
\omega=\sum_{i=1}^{k} f_{2 i-1} \wedge f_{2 i}
$$

Além disso, $\omega$ é não degenerada se, e só se, $2 k=m$.

Demonstração. Se $\omega=0$ basta tomar $k=0$. Considere $\omega \neq 0$. Por indução, assuma o resultado provado para $\operatorname{dim} V \leq n-1$. Considere agora $\left\{v_{1}, \ldots, v_{2 k}, v_{2 k+1}, \ldots, v_{n}\right\}$ base de $V$ e sua base dual $\left\{v_{1}^{*}, \ldots, v_{2 k}^{*}, v_{2 k+1}^{*}, \ldots, v_{n}^{*}\right\}$. Podemos escrever

$$
\omega=\sum_{i<j} a_{i j} v_{i}^{*} \wedge v_{j}^{*}
$$

Sem perda de generalidade, podemos assumir que $a_{12} \neq 0$. Defina então os funcionais

$$
\begin{aligned}
f_{1} & =\frac{1}{a_{12}} \iota_{v_{1}} \omega \\
f_{2} & =\iota_{v_{2}} \omega
\end{aligned}
$$

e a forma $\omega_{1}=\omega-f_{1} \wedge f_{2}$. Temos então que

$$
\begin{aligned}
\iota_{v_{1}} \omega_{1} & =\iota_{v_{1}} \omega-\left(\iota_{v_{1}} f_{1}\right) \wedge f_{2}+f_{1} \wedge\left(\iota_{v_{1}} f_{2}\right) \\
& =\iota_{v_{1}} \omega-\left(\frac{1}{a_{12}} \iota_{v_{1}} \iota_{v_{1}} \omega\right) \wedge f_{2}+f_{1} \wedge\left(\iota_{v_{1}} \iota_{v_{2}} \omega\right) \\
& =a_{12} f_{1}+f_{1} \wedge\left(-a_{12}\right) \\
& =0
\end{aligned}
$$


e também

$$
\begin{aligned}
\iota_{v_{2}} \omega_{1} & =\iota_{v_{2}} \omega-\left(\iota_{v_{2}} f_{1}\right) \wedge f_{2}+f_{1} \wedge\left(\iota_{v_{2}} f_{2}\right) \\
& =\iota_{v_{1}} \omega-\left(\frac{1}{a_{12}} \iota_{v_{2}} \iota_{v_{1}} \omega\right) \wedge f_{2}+f_{1} \wedge\left(\iota_{v_{2}} \iota_{v_{2}} \omega\right) \\
& =f_{2}+1 \wedge f_{2} \\
& =f_{2}-f_{2} \wedge 1 \\
& =0
\end{aligned}
$$

Podemos então considerar $\omega_{1}$ como uma forma apenas em $W$ o subespaço gerado por $\left\{v_{3}, \ldots, v_{n}\right\}$. Mas, pela hipótese de indução, existe $\left\{f_{3}, \ldots, f_{2 k}, f_{2 k+1}, \ldots, f_{n}\right\}$ tal que

$$
\omega_{1}=\sum_{i=3}^{k} f_{2 i-1} \wedge f_{2 i}
$$

o que nos dá

$$
\omega=\sum_{i=1}^{k} f_{2 i-1} \wedge f_{2 i}
$$

Observe, no entanto, que $f_{1}\left(v_{2}\right)$ e $f_{2}\left(v_{1}\right)$ são não nulos, $\operatorname{logo}\left\{f_{1}, f_{2}, v_{3}^{*}, \ldots, v_{n}^{*}\right\}$ é uma base de $V^{*}$, o que por sua vez implica que $\left\{f_{1}, \ldots, f_{n}\right\}$ também é base de $V^{*}$, provando a primeira parte do lema.

Para provar a segunda parte, note que, se $2 k<m$, temos que $\iota_{f_{m}} \omega=0$ e $\omega$ é degenerada. Por outro lado, se $2 k=m$, seja $\left\{w_{1}, \ldots, w_{2 k}\right\}$ base de $V$ tal que $\left\{f_{1}, \ldots, f_{n}\right\}$ é sua base dual. Então $\iota_{w_{2 i-1}} \omega=f_{2 i}$ e $\iota_{w_{2 i}} \omega=-f_{2 i-1}$ são não nulos, $\log \omega \omega$ é não degenerada.

Podemos agora provar o resultado desejado.

Lema 1.2.8 (Darboux linear). Seja $V$ um espaço vetorial e $\omega$ uma forma simpética em $V$. Existe $\psi: \mathbb{R}^{2 n} \rightarrow V$ tal que $\psi^{*} \omega$ é a forma simplética canôninca.

Demonstração. Pelo lema 1.2.7, existe $\left\{f_{1}, \ldots, f_{2 n}\right\}$ base de $V^{*}$ tal que

$$
\omega=\sum_{i=1}^{n} f_{2 i-1} \wedge f_{2 i}
$$

. Considere $\left\{v_{1}, \ldots, v_{2 n}\right\}$ base de $V$ tal que $\left\{f_{1}, \ldots, f_{2 n}\right\}$ é sua base dual e defina $\psi: \mathbb{R}^{2 n} \rightarrow V$ tal que $\psi\left(e_{i}\right)=v_{2 i-1}$ e $\psi\left(e_{i+n}\right)=v_{2 i}$, onde $\left\{e_{1}, \ldots, e_{2 n}\right\}$ é a base canônica de $\mathbb{R}^{2 n}$.

Se $\left\{e_{1}^{*}, \ldots, e_{2 n}^{*}\right\}$ é a base dual de $\left\{e_{1}, \ldots, e_{2 n}\right\}$, temos que $\psi^{*}\left(f_{2 i-1}\right)=e_{i}^{*}$ e $\psi^{*}\left(v_{2 i}^{*}\right)=e_{i+n}^{*}$. Assim

$$
\begin{aligned}
\psi^{*} \omega & =\sum_{i=1}^{n} \psi^{*}\left(f_{2 i-1}\right) \wedge \psi^{*}\left(f_{2 i}\right) \\
& =\sum_{i=1}^{n} e_{i}^{*} \wedge e_{i+n}^{*}
\end{aligned}
$$

Para provar o caso geral, usaremos o chamado truque de Moser, que garante que, dada uma família de formas simpléticas, sob certas condições, todas as formas são equivalentes. Para provar isso usaremos um pequeno lema técnico sobre formas diferenciáveis. 
Lema 1.2.9. Sejam $M$ variedade diferenciável e uma família $\omega_{t} \in \Omega^{k}(M)$ de formas diferenciáveis. Dadas uma família de campos $X_{t} \in \mathfrak{X}(M)$ então existe uma família $\rho_{t}: M \rightarrow M$ de difeomorfismos com $\rho_{0}=\mathrm{Id}$ tais que

$$
\frac{\mathrm{d}}{\mathrm{d} t} \rho_{t}^{*} \omega_{t}=\rho_{t}^{*}\left(\mathcal{L}_{X_{t}}+\frac{\mathrm{d} \omega_{t}}{\mathrm{~d} t}\right)
$$

Lema 1.2.10 (Truque de Moser). Seja $M$ variedade compacta e $\omega_{t}$ família de formas simpléticas tal que $\frac{\mathrm{d} \omega_{t}}{\mathrm{~d} t}$ é exata para todo $t$. Então existe uma familia de difeomorfismos $\rho_{t}: M \rightarrow M$ com $\rho_{0}=\operatorname{Id}_{M}$ tal que $\rho_{t}^{*} \omega_{t}=\omega_{0}$.

Demonstração. Seja $\sigma_{t} \in \Omega^{1}(M)$ família de 1-formas tal que

$$
\frac{\mathrm{d} \omega_{t}}{\mathrm{~d} t}=\mathrm{d} \sigma_{t}
$$

Como $\omega_{t}$ é não-degenerada, podemos encontrar campos $X_{t} \in \mathfrak{X}(M)$ tais que

$$
\iota_{X} \omega_{t}=-\sigma_{t}
$$

Como $\omega_{t}$ é fechada, o lema 1.2.9 nos garante que existe uma $\rho_{t}: M \rightarrow M$ familia de difeomorfismos com $\rho_{0}=\operatorname{Id}_{M}$ satisfazendo

$$
\begin{aligned}
\frac{\mathrm{d}}{\mathrm{d} t} \rho_{t}^{*} \omega_{t} & =\rho_{t}^{*}\left(\mathcal{L}_{X_{t}}+\frac{\mathrm{d} \omega_{t}}{\mathrm{~d} t}\right) \\
& =\rho_{t}^{*}\left(\mathrm{~d} \iota_{X_{t}} \omega_{t}+\mathrm{d} \sigma_{t}\right) \\
& =0
\end{aligned}
$$

o que implica $\rho_{t}^{*} \omega_{t}=\rho_{0}^{*} \omega_{0}=\omega_{0}$.

Teorema 1.2.11 (Darboux). Toda variedade simplética é localmente simplectomorfa ao $\mathbb{R}^{2 n}$ munido da forma simplética canônica.

Demonstração. Seja $(M, \omega)$ variedade simplética e $p \in M$. Pelo teorema 1.2 .8 , existe $F: \mathbb{R}^{2 n} \rightarrow$ $T_{p} M$ tal que $F^{*}\left(\omega_{p}\right)$ é a forma simplética canônica do $\mathbb{R}^{2 n}$.

Seja então $\psi: U \subset \mathbb{R}^{2 n} \rightarrow V \subset M$ tal que $U$ é contrátil, $p \in V$ e $\left.\psi\right|_{T_{p} M}=F$. Considere $\omega_{0}$ a forma simplética canônica de $\mathbb{R}^{n}$ restrita a $V$ e $\omega_{1}=\psi^{*} \omega$ e defina a família de 2-formas

$$
\omega_{t}=t \omega_{1}+(1-t) \omega_{0}
$$

Vamos mostrar que $\omega_{t}$ é simplético para todo $t \in[0,1]$. É suficiente mostrar que $\omega_{t}$ é fechado e não degenerado. Para verificar que $\omega_{t}$ é fechado basta ver

$$
\begin{aligned}
\mathrm{d} \omega_{t} & =t \mathrm{~d} \omega_{1}+(1-t) \mathrm{d} \omega_{0} \\
& =0
\end{aligned}
$$

Para ver que $\omega_{t}$ é não degenerada, note que $\left(\omega_{1}\right)_{\psi^{-1}(p)}=\omega_{0}, \operatorname{logo}\left(\omega_{t}\right)_{\psi^{-1}(p)}=\omega_{0}$ é não degenerada. Assim, reduzindo $U$ se necessário, temos que $\omega_{t}$ é não degenerada em todo ponto.

Como $U$ é contrátil e $\mathrm{d}\left(\omega_{1}-\omega_{0}\right)=0$, existe $\sigma \in \Omega^{1}(U)$ tal que $\frac{\mathrm{d}}{\mathrm{d} t} \omega_{t}=\omega_{1}-\omega_{0}=\mathrm{d} \sigma$.

O resultado segue pelo truque de Moser.

\section{Outras Definições}

Veremos que podemos adaptar vários conceitos da geometria Riemmaneana para a geometria simplética. O primeiro que apresentaremos é o de espaço complementar.

Definição 1.2.12. Sejam $(V, \omega)$ espaço simplético e $W$ subespaço vetorial de $V$. Então o complementar simplético de $W$ é definido por

$$
W^{\omega}:=\{v \in V \mid \omega(v, w)=0, \forall w \in W\}
$$


Podemos classificar subespaços e subvariedades de acordo com os complementares simpléticos. Sejam $(V, \omega)$ é um espaço simplético e $W$ é um subespaço de $V$. $W$ é dito subespaço isotrópico se $W \subset W^{\omega}$ e coisotrópico se $W^{\omega} \subset W$. Um subespaço isotrópico e coisotrópico é dito Lagrangeano. Finalmente $W$ é dito simplético se $W \cap W^{\omega}=\emptyset$.

Analogamente, se $(M, \omega)$ é uma variedade simplética e $N$ é subvariedade de $M$, temos que $N$ é isotrópica, coisotrópica, Lagrangeana ou simplética se, para todo $p \in N, T_{p} N$ é um subespaço isotrópico, coisotrópico, Lagrangeano ou simplético de $\left(T_{p} M, \omega_{p}\right)$

Proposição 1.2.13. Seja $(V, \omega)$ espaço simplético e $W$ subespaço vetorial de $V$. Então temos que $\operatorname{dim} W+\operatorname{dim} W^{\omega}=\operatorname{dim} V$.

Demonstração. Considere $\varphi: V \rightarrow V^{*}$ a função linear dada por $\varphi(v)(w)=\omega(v, w)$. Como $\omega$ é não degenerada, $\varphi$ é um isomorfismo. Observe que $\varphi\left(W^{\omega}\right)=W^{0}$ é o anulador de $W$. Logo $\operatorname{dim} W^{\omega}=\operatorname{dim} W^{0}=\operatorname{dim} V-\operatorname{dim} W$.

Na proposição a seguir tentaremos reproduzir outro conceito da geometria Riemanneana: o gradiente. Recorde que, dado um produto interno $g$ em $M$ e uma função suave $f: M \rightarrow \mathbb{R}$, grad $f$ é o campo vetorial tal que $\mathrm{d} f=g(\operatorname{grad} f, \cdot)$.

Proposição 1.2.14. Se $M$ uma variedade simplética, dada $f: M \rightarrow \mathbb{R}$ função suave, existe um único campo $X_{f} \in \mathfrak{X}(M)$ tal que $\mathrm{d} f=\omega\left(X_{f}, \cdot\right)$. $X_{f}$ é chamado gradiente simplético de $f$.

Demonstração. Vamos primeiro provar a unicidade do gradiente simplético. Suponha que existam $X_{1}, X_{2} \in \mathfrak{X}(M)$ satisfazendo as propriedades do gradiente simplético. Temos então, para todo $Y \in \mathfrak{X}(M)$

$$
\begin{aligned}
\omega\left(X_{1}-X_{2}, Y\right) & =\omega\left(X_{1}, Y\right)-\omega\left(X_{2}, Y\right) \\
& =\mathrm{d} f(Y)-\mathrm{d} f(Y) \\
& =0
\end{aligned}
$$

Como $\omega$ é não-degenerada e $Y$ é qualquer, temos $X_{1}=X_{2}$.

A existência do gradiente simplético vem do fato de $\omega$ ser não degenerada.

Lema 1.2.15. Dada $(M, \omega)$ variedade simplética, a aplicação $C^{\infty}(M) \ni f \mapsto X_{f} \in \mathfrak{X}(M)$ é linear.

Demonstração. Sejam $f_{1}, f_{2}: M \rightarrow \mathbb{R}$ e $k_{1}, k_{2} \in \mathbb{R}$. Então, para todo $p \in M$ e $v \in T_{p} M$, temos que

$$
\begin{aligned}
\omega\left(X_{k_{1} f_{1}+k_{2} f_{2}}, v\right) & =\mathrm{d}\left(k_{1} f_{1}+k_{2} f_{2}\right)_{p}(v) \\
& =\left(k_{1} \mathrm{~d} f_{1}+k_{2} \mathrm{~d} f_{2}\right)_{p}(v) \\
& =\omega\left(k_{1} X_{f_{1}}+k_{2} X_{f_{2}}, v\right)
\end{aligned}
$$

O resultado vem da não-degenerescência de $\omega$.

\subsubsection{Equações de Hamilton}

Nesta seção tentaremos reescrever o formalismo Lagrangeano sob a luz da geometria simplética, transportando-o para o fibrado cotangente. A idéia é reduzir o sistema de equações diferenciais de segunda ordem dado pelas equações de Euler-Lagrange para um de primeira ordem. Essas equações, conhecidas como as equações de Hamilton, são definidas a partir de uma função $H: \mathbb{R}^{2 n} \rightarrow \mathbb{R}$, que chamaremos de Hamiltoneano, da seguinte forma

$$
\begin{aligned}
q_{i}^{\prime}(t) & =\frac{\partial H}{\partial p_{i}}(q(t), p(t)) \\
p_{i}^{\prime}(t) & =-\frac{\partial H}{\partial q_{i}}(q(t), p(t))
\end{aligned}
$$


onde $p, q:(-\epsilon, \epsilon) \rightarrow \mathbb{R}^{n}$ são curvas, $\frac{\partial H}{\partial q_{i}}$ denota a derivada parcial de $H$ com relação à $i$-ésima coordenada e $\frac{\partial H}{\partial p_{i}}$ denota a derivada parcial com relação à $(n+i)$-ésima coordenada. Dizemos, nesse caso, que a curva $(q, p)$ satisfaz as equações de Hamilton para $H$ ou que $(q, p)$ é solução das equações de Hamilton para $H$.

Usaremos esse sistema de equações para tentar encontrar condições equivalentes às equações de Euler-Lagrange. Entretanto isso não será possível para todo Lagrangeano. Vamos começar então definindo uma sub-classe de Lagrangeanos

Definição 1.2.16. Um Lagrangeano $L$ é dito regular se a matriz $\left[\frac{\mathrm{d}^{2} L}{\mathrm{~d} \dot{q}^{2}}\right]$ é sempre invertível. $L$ é dito hiper-regular se sua transformação de Legendre $T_{L}$ é um difeomorfismo.

Note que se $L$ é hiper-regular, então

$$
\mathrm{d} T_{L}=\left[\begin{array}{cc}
\mathrm{Id} & 0 \\
\frac{\mathrm{d}^{2} L}{\mathrm{~d} q \mathrm{~d} \dot{q}} & \frac{\mathrm{d}^{2} L}{\mathrm{~d} \dot{q}^{2}}
\end{array}\right]
$$

é sempre invertível. Logo todo Lagrangeano hiper-regular também é regular.

Definição 1.2.17. Para um Lagrangeano hiper-regular $L: \mathrm{T} M \rightarrow \mathbb{R}$, podemos definir seu Hamiltoneano associado como a função $H_{L}=E_{L} \circ T_{L}^{-1}$. Em particular, quando $M=\mathbb{R}^{n}$, procurar soluções das equações de Hamilton para $H_{L}$.

A proposição a seguir se vale desse fato para tentar transportar os resultados da mecânica Lagrangeana para o fibrado cotangente via a transformação de Legendre, primeiramente no caso $M=\mathbb{R}^{n}$.

Proposição 1.2.18. Seja $L: \mathbb{R}^{2 n} \rightarrow \mathbb{R}$ lagrangeano hiper-regular. Então $\alpha:[0,1] \rightarrow \mathbb{R}^{n}$ é solução de Euler-Lagrange em coordenadas para $L$ se, e só se, $T_{L}\left(\alpha, \alpha^{\prime}\right)$ é solução das equações de Hamilton para $H_{L}$.

Demonstração. Defina a curva $\beta:[0,1] \rightarrow \mathbb{R}^{n}$ tal que

$$
\begin{aligned}
(\alpha(t), \beta(t)) & =T_{L}\left(\alpha(t), \alpha^{\prime}(t)\right) \\
& =\left(\alpha(t), \frac{\partial L}{\partial \dot{q}}\left(\alpha(t), \alpha^{\prime}(t)\right)\right)
\end{aligned}
$$

As equações de Hamilton para $H_{L}$ então se escrevem como

$$
\alpha_{i}^{\prime}(t)=\frac{\partial H_{L}}{\partial p_{i}}(\alpha(t), \beta(t)) \beta_{i}^{\prime}(t)=-\frac{\partial H_{L}}{\partial q_{i}}(\alpha(t), \beta(t))
$$

Vamos buscar uma expressão explícita para as derivadas de $H_{L}$. Pela proposição 1.1.13, temos que $T_{L}(q, \dot{q})=\left(q, \frac{\partial L}{\partial \dot{q}}(q, \dot{q})\right)$. Logo podemos, com um pequeno abuso de notação, econtrar uma função $\dot{q}: \mathbb{R}^{2 n} \rightarrow \mathbb{R}^{n}$ tal que $T_{L}^{-1}(q, p)=(q, \dot{q}(q, p))$. Com isso conseguimos escrever

$$
\begin{aligned}
H_{L}(q, p) & =E_{L}(q, \dot{q}(q, p)) \\
& =\sum_{i=1}^{n} \frac{\partial L}{\partial \dot{q}_{i}}(q, \dot{q}(q, p)) \dot{q}_{i}(q, p)-L(q, \dot{q}(q, p)) \\
& =\sum_{i=1}^{n} p_{i} \dot{q}_{i}(q, p)-L(q, \dot{q}(q, p))
\end{aligned}
$$


Temos então que

$$
\begin{aligned}
\frac{\partial H_{L}}{\partial p_{j}}(q, p) & =\sum_{i=1}^{n} \frac{\partial p_{i}}{\partial p_{j}} \dot{q}_{i}(q, p)+\sum_{i=1}^{n} p_{i} \frac{\partial q_{i}}{\partial p_{j}}(q, p)-\sum_{i=0}^{n} \frac{\partial L}{\partial \dot{q}_{i}}(q, \dot{q}(q, p)) \frac{\partial \dot{q}_{i}}{\partial p_{j}}(q, p) \\
& =\dot{q}_{i}(q, p)+\sum_{i=1}^{n}\left(p_{i}-\frac{\partial L}{\partial \dot{q}_{i}}(q, \dot{q}(q, p))\right) \frac{\partial \dot{q}_{i}}{\partial p_{j}}(q, p) \\
& =\dot{q}_{i}(q, p) \\
\frac{\partial H_{L}}{\partial q_{j}}(q, p) & =\sum_{i=1}^{n} p_{i} \frac{\partial \dot{q}_{i}}{q_{j}}(q, p)-\frac{\partial L}{\partial q_{i}}(q, \dot{q}(q, p))-\sum_{i=1}^{n} \frac{\partial L}{\partial \dot{q}_{i}}(q, \dot{q}(q, p)) \frac{\partial \dot{q}_{i}}{\partial q_{j}}(q, p) \\
& =-\frac{\partial L}{\partial q_{i}}(q, \dot{q}(q, p))+\sum_{i=1}^{n}\left(p_{i}-\frac{\partial L}{\partial \dot{q}_{i}}(q, \dot{q}(q, p))\right) \frac{\partial \dot{q}_{i}}{\partial q_{j}}(q, p) \\
& =-\frac{\partial L}{\partial q_{i}}(q, \dot{q}(q, p))
\end{aligned}
$$

Assim, os termos à direita das equações de Hamilton são obtidos substituindo $(q, p)$ por $(\alpha(t), \beta(t))$ em 1.11 e 1.13. Dessa forma obtemos

$$
\begin{aligned}
& \frac{\partial H_{L}}{\partial p_{i}}(\alpha(t), \beta(t))=\dot{q}_{i}(\alpha(t), \beta(t)) \\
& \frac{\partial H_{L}}{\partial q_{i}}(\alpha(t), \beta(t))=-\frac{\partial L}{\partial q_{i}}(q, \dot{q}(\alpha(t), \beta(t)))
\end{aligned}
$$

Note que $\dot{q}_{i}(\alpha(t), \beta(t))=\alpha^{\prime}(t)$ para toda curva $\alpha$ com $\beta$ definido como acima, fazendo com que a primeira metade das equações de Hamilton sempre seja satisfeita. Temos então que $(\alpha(t), \beta(t))$ seja solução das equações de Hamilton para $H_{L}$ se, e só se

$$
\begin{aligned}
\frac{\partial L}{\partial q_{i}}(q, \dot{q}(\alpha(t), \beta(t))) & =\beta_{i}^{\prime}(t) \\
& =\frac{\mathrm{d}}{\mathrm{d} t} \frac{\partial L}{\partial \dot{q}_{i}}\left(\alpha(t), \alpha^{\prime}(t)\right)
\end{aligned}
$$

que é exatamente a condição para que $\alpha$ seja solução de Euler-Lagrange em coordenadas para $L$.

Observação 1.2.19. Para transportar esse resultado para variedades mais gerais, seria interessante relacionar as equações de Hamilton com um conceito que não dependa de coordenadas. Note que dizer que $(q, p)$ é solução das equações de Hamilton para um Hamiltoneano $H$ é equivalente a dizer que $(q, p)$ é solução do fluxo gerado pelo campo $X=\left(\frac{\partial H}{\partial p},-\frac{\partial H}{\partial q}\right)$. Usando $\omega$ a forma simplética canônica de $\mathbb{R}^{2 n}$ temos que $X=X_{H}$ o gradiente simplético de $H$.

Com isso em mente, queremos relacionar soluções de Euler-Lagrange com soluções de fluxos Hamiltoneanos em variedades Riemanneanas quaisquer. Para isso precisaremos do seguinte lema técnico.

Lema 1.2.20. Sejam $\left(M, \omega_{M}\right),\left(N, \omega_{N}\right)$ variedade simpléticas e $f: M \rightarrow N$ simplectomorfismo. Se $H_{N}: N \rightarrow \mathbb{R}$ é Hamiltoneano e $H_{M}=H_{N} \circ f$, temos que $\alpha:[0,1] \rightarrow M$ é solução do fluxo de $X_{H_{M}}$ se, e só se, $f \circ \alpha$ é solução do fluxo de $X_{H_{N}}$ 
Demonstração. Observe que, dado $p \in M$ e $v \in T_{p} M$

$$
\begin{aligned}
\omega_{M}\left(X_{H_{M}}(p), v\right) & =\mathrm{d}\left(H_{M}\right)_{p} \\
& =\mathrm{d}\left(H_{N} \circ f\right)_{p} \\
& =\mathrm{d}\left(H_{N}\right)_{f(p)} \circ \mathrm{d} f_{p} \\
& =\omega_{N}\left(X_{H_{N}} \circ f(p), \mathrm{d} f_{p}(v)\right) \\
& =\omega_{M}\left(\mathrm{~d} f_{p}^{-1} \circ X_{H_{M}} \circ f(p), v\right)
\end{aligned}
$$

onde a última igualdade vem do fato de que $f$ é um simplectomorfismo.

Como $\omega_{M}$ é não-degenerada, temos que

$$
X_{H_{M}}(p)=\mathrm{d} f_{p}^{-1} \circ X_{H_{N}} \circ f(p)
$$

Dessa forma, dada uma curva $\alpha:[0,1] \rightarrow M$, temos que se $X_{H_{N}}(f \circ \alpha)=(f \circ \alpha)^{\prime}$, então

$$
\begin{aligned}
X_{H_{M}}(\alpha(t)) & =\mathrm{d} f_{\alpha(t)}^{-1} \circ X_{H_{M}}((f \circ \alpha)(t)) \\
& =\mathrm{d} f_{\alpha(t)}^{-1} \circ(f \circ \alpha)^{\prime}(t) \\
& =\alpha^{\prime}(t)
\end{aligned}
$$

A outra implicação é obtida fazendo o mesmo para $f^{-1}$.

Proposição 1.2.21. Seja $M$ uma variedade Riemanneana e $L: \mathrm{T} M \rightarrow \mathbb{R}$ um Lagrangeano. Então $\alpha:[0,1] \rightarrow M$ é solução de Euler-Lagrange em coordenadas se, e só se, $T_{L}\left(\alpha^{\prime}\right)$ é solução do fluxo Hamiltoneano de $H_{L}$.

Demonstração. A idéia é usar a proposição 1.2 .18 e a comutatividade do diagrama abaixo, provada em 1.1.13.

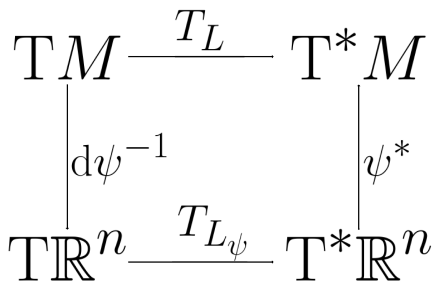

Figura 1.2: Diagrama transformação de Legendre e coordenadas

Sejam $\omega$ a forma simplética natural de $\mathrm{T}^{*} M$ e $\psi: U \subset \mathbb{R}^{n} \rightarrow V \subset M$ carta de $M$. Defina então $\tilde{\alpha}=\psi^{-1} \circ \alpha$ e $L_{\psi}=L \circ \mathrm{d} \psi$. Como as equações de Euler-Lagrange são invariantes por difeomorfismos, temos que $\alpha$ é solução de Euler-Lagrange para $L$ se, e só se, $\tilde{\alpha}$ é solução de Euler-Lagrange para $L_{\psi}$, o que, pela proposição 1.2.18 e observação 1.2.19 é equivalente a $T_{L_{\psi}}\left(\tilde{\alpha}, \tilde{\alpha}^{\prime}\right)$ ser solução do fluxo de $X_{H_{L_{\psi}}}$.

Logo, basta provar que $T_{L_{\psi}}\left(\tilde{\alpha}, \tilde{\alpha}^{\prime}\right)$ é solução do fluxo de $X_{H_{L_{\psi}}}$ se, e só se, $T_{L}\left(\alpha^{\prime}\right)$ é solução do fluxo de $X_{H_{L}}$

Mas observe que, dado $v \in \mathrm{TR}^{n}$

$$
\begin{aligned}
E_{L_{\psi}}(v) & =T_{L_{\psi}}(v)(v)-L_{\psi}(v) \\
& =T_{L}(\mathrm{~d} \psi(v))(\mathrm{d} \psi(v))-L(\mathrm{~d} \psi(v)) \\
& =E_{L} \circ \mathrm{d} \psi(v)
\end{aligned}
$$


e, portanto

$$
\begin{aligned}
H_{L_{\psi}} & =E_{L_{\psi}} \circ T_{L_{\psi}}^{-1} \\
& =E_{L} \circ \mathrm{d} \psi \circ\left(\psi^{*} \circ T_{L} \circ \mathrm{d} \psi\right) \\
& =E_{L} \circ \mathrm{d} \psi \circ \mathrm{d} \psi^{-1} \circ T_{L}^{-1} \circ\left(\psi^{*}\right)^{-1} \\
& =H_{L} \circ\left(\psi^{*}\right)^{-1}
\end{aligned}
$$

Mas note que, como mostrado no exemplo 1.2.4, se escrevermos $\mathrm{d} \psi^{-1}=\left(q_{1}, \ldots, q_{n}, p_{1}, \ldots, p_{n}\right)$ e fizermos $\omega$ a forma simplética canônica de $\mathrm{T}^{*} M$ e $\omega_{0}$ a forma simplética canônica de $\mathbb{R}^{2 n}$, temos $\left(\left(\psi^{*}\right)^{-1}\right)^{*} \omega=\sum_{i=1}^{n} \mathrm{~d} q_{i} \wedge \mathrm{d} p_{i}=\omega_{0}$. Logo $\left(\psi^{*}\right)^{-1}$ é um simplectomorfismo entre $\left(\mathrm{T}^{*} M, \omega\right)$ e $\left(\mathbb{R}^{2 n}, \omega_{0}\right)$.

A solução segue pelo lema 1.2.20.

Corolário 1.2.22. Seja $M$ variedade Riemanneana, $\omega$ a forma simplética canônica de $\mathrm{T}^{*} M$. Sejam também $L: \mathrm{T} M \rightarrow \mathbb{R}$ um Lagrangeano e $\omega_{L}=T_{L}^{*} \omega$. Então uma curva $\alpha:[0,1] \rightarrow M$ é solução de Euler-Lagrange em coordenadas para $L$ se, e só se, $\alpha^{\prime}$ é solução do fluxo Hamiltoneano do funcional energia $E_{L}$ referente à forma simplética $\omega_{L}$

Demonstração. Note que $T_{L}$ é um simplectomorfismo entre as variedades simpléticas $\left(\mathrm{T} M, \omega_{L}\right)$ e $\left(\mathrm{T}^{*} M, \omega\right)$. Recordando que $E_{L}=H_{L} \circ T_{L}$, o lema 1.2 .20 nos garante que, dada uma curva $\alpha:[0,1] \rightarrow M, \alpha^{\prime}$ é solução do fluxo de $X_{E_{L}}$ se, e só se, $T_{L}\left(\alpha^{\prime}\right)$ é solução do fluxo de $X_{H_{L}}$. A proposição 1.2.21 conclui a prova.

\subsubsection{Métrica de Jacobi}

Agora temos o arcabouço necesário para provar o teorema 1.1.12. Para isso será necessário provar dois lemas. O primeiro é um resultado bastante técnico, mas que apresenta o argumento central da demonstração.

Lema 1.2.23. Sejam $(M, \omega)$ uma variedade simplética e $H_{1}, H_{2}: M \rightarrow \mathbb{R}$ Hamiltoneanos em $M$ tais que existem $h_{1}$ e $h_{2}$ valores regulares de $H_{1}$ e $H_{2}$, respectivamente, com $H_{1}^{-1}\left(h_{1}\right)=H_{2}^{-1}\left(h_{2}\right)$. Então se $\alpha_{1}$ e $\alpha_{2}$ são soluções dos campos $X_{H_{1}}$ e $X_{H_{2}}$, temos que existe reparametrização $\varphi:[0,1] \rightarrow[0,1]$ tal que $\alpha_{2}=\alpha_{1} \circ \varphi$.

Demonstração. Defina $\Lambda:=H_{1}^{-1}\left(h_{1}\right)=H_{2}^{-1}\left(h_{2}\right)$. Se $z \in \Lambda$ e $v \in \mathrm{T}_{z} \Lambda$ então temos que

$$
\begin{aligned}
0 & =\mathrm{d}\left(H_{1}\right)_{z}(v) \\
& =\omega\left(X_{H_{1}}(z), v\right)
\end{aligned}
$$

Isso implica que $X_{H_{1}}(z) \in\left(\mathrm{T}_{z} \Lambda\right)^{\omega}$. O mesmo vale para $H_{2}$.

Pela proposição 1.2.13, temos que $\operatorname{dim} \mathrm{T}_{z} \Lambda+\operatorname{dim}\left(\mathrm{T}_{z} \Lambda\right)^{\omega}=\operatorname{dim} M$. Juntando a isso o fato de que $\operatorname{dim} \mathrm{T}_{z} \Lambda=\operatorname{dim} M-1$, temos que $\operatorname{dim}\left(\mathrm{T}_{z} \Lambda\right)^{\omega}=1$. Além disso, o fato de $h_{1}$ e $h_{2}$ serem valores regulares implica que $X_{H_{1}}$ e $X_{H_{2}}$ são campos que não de anulam em $\Lambda$ e que estão ambops contidos no mesmo subespaço unidimensional. Assim, podemos encontrar uma função suave $\lambda: \Lambda \rightarrow \mathbb{R}$ tal que, para todo $z \in \Lambda$

$$
X_{H_{2}}(z)=\lambda(z) X_{H_{1}}(z)
$$

Vamos agora construir a reparametrização de $\alpha_{1}$ para $\alpha_{2}$. Defina a função $f: \mathbb{R} \rightarrow \mathbb{R}$ por $f(t)=\int_{0}^{t} \frac{1}{\lambda \circ \alpha_{1}(s)} \mathrm{d} s$. Como $f^{\prime}(t)=\frac{1}{\lambda \circ \alpha_{1}(t)} \neq 0$, temos que $f$ é inversível. Vamos provar que $\varphi=f^{-1}$ é a reparametrização desejada.

De fato, temos que 


$$
\begin{aligned}
\varphi^{\prime}(t) & =\left(f^{-1}\right)^{\prime}(t) \\
& =\frac{1}{f^{\prime}\left(f^{-1}(t)\right)} \\
& =\left(\lambda \circ \alpha_{1}\right)(\varphi(t))
\end{aligned}
$$

Isso implica que

$$
\begin{aligned}
\left(\alpha_{1} \circ \varphi\right)^{\prime}(t) & =\alpha_{1}^{\prime}(\varphi(t)) \varphi^{\prime}(t) \\
& =X_{H_{1}}\left(\alpha_{1} \circ \varphi(t)\right) \lambda\left(\alpha_{1} \circ \varphi(t)\right) \\
& =X_{H_{2}}\left(\alpha_{1}(\varphi(t))\right)
\end{aligned}
$$

ou seja, que $\alpha_{1} \circ \varphi$ é solução do fluxo de $X_{H_{2}}$. Mas, pela unicidade das souleções de equações diferenciais ordinárias, temos que $\alpha_{1} \circ \varphi=\alpha_{2}$.

O segundo lema consiste apenas dos cálculos dos Hamiltoneanos associados aos Lagrangeanos apresentados no teorema.

Lema 1.2.24. Sejam $L, L_{J}: \mathrm{T} M \rightarrow \mathbb{R}$ os Lagrangeanos apresentados no teorema e denote por $H, H_{J}: \mathrm{T}^{*} M \rightarrow \mathbb{R}$ os respectivos Hamiltoneanos associados. Temos então que

$$
\begin{aligned}
H\left(v_{p}\right) & =\frac{1}{2}\left\|v_{p}\right\|^{2}+U(q) \\
H_{J}\left(v_{p}\right) & =\frac{\left\|v_{p}\right\|^{2}}{2(c-U(q))}
\end{aligned}
$$

Agora podemos demonstrar o teorema.

Demonstração. Se provarmos que $H^{-1}(c)=H_{J}^{-1}(1)$ e $c$ e 1 são valores regulares de $H$ e $H_{J}$, respectivamente, pelo lema 1.2.23 o teorema estará provado.

A igualdade das pré-imagens pode ser provada tomando $v_{p} \in \mathrm{T}_{p}^{*} M$ e observando que

$$
\begin{aligned}
H\left(v_{p}\right)=c & \Leftrightarrow\left\|v_{p}\right\|^{2}=2(c-U(q)) \\
& \Leftrightarrow \frac{\left\|v_{p}\right\|^{2}}{2(c-U(q))}=1 \\
& \Leftrightarrow H_{J}\left(v_{p}\right)=1
\end{aligned}
$$

Para provar que $c$ e 1 são valores regulares, note que, como o contra-domínio de $H$ funções é $\mathbb{R}$, basta encontrar uma curva $\alpha:(-\epsilon, \epsilon) \rightarrow \mathrm{T}^{*} M$ tal que $H(\alpha(0))=c$ e $\left.\frac{\mathrm{d}}{\mathrm{d} t} H((\alpha(t)))\right|_{t=0} \neq 0$. O mesmo vale para $H_{J}$, mas fazendo $H_{J}(\alpha(0))=1$.

Considere $v_{p} \in \mathrm{T}_{p}^{*} M$ e defina $\alpha(t)=(t+1) v_{p}$ para todo $t \in(-\epsilon, \epsilon)$. Se escolhermos $v_{p}$ tal que $H\left(v_{p}\right)=c$, teremos que $\left\|v_{p}\right\|^{2}=2(c-U(q)) \neq 0 \mathrm{e}$

$$
\begin{aligned}
\left.\frac{\mathrm{d}}{\mathrm{d} t} H((\alpha(t)))\right|_{t=0} & =\left.\frac{\mathrm{d}}{\mathrm{d} t}\left(\frac{1}{2}\left\|(t+1) v_{p}\right\|^{2}+U(q)\right)\right|_{t=0} \\
& =\frac{1}{2}\left\|v_{p}\right\|^{2} \\
& \neq 0
\end{aligned}
$$

$\operatorname{logo} c$ é valor regular de $H$. 
Para provar que 1 é valor regular de $H_{J}$, escolha $v_{p}$ tal que $H_{J}\left(v_{p}\right)=1$. Dessa forma temos $\left\|v_{P}\right\|^{2}=2(c-U(q))$ e, usando $\alpha$ como definido acima

$$
\begin{aligned}
\left.\frac{\mathrm{d}}{\mathrm{d} t} H_{J}((\alpha(t)))\right|_{t=0} & =\left.\frac{\mathrm{d}}{\mathrm{d} t} \frac{(t+1)\left\|v_{p}\right\|^{2}}{2(c-U(q))}\right|_{t=0} \\
& =\frac{\left\|v_{p}\right\|^{2}}{2(c-U(q))} \\
& \neq 0
\end{aligned}
$$




\section{Capítulo 2}

\section{Fatos Básicos e Ações simpléticas}

\subsection{Introdução}

Problemas matemáticos muitas vezes apresentam simetrias, ou seja, eles tem mais informação do que o necessário. Essas simetrias podem ser originais do problema ou surgir após a introdução de uma estrutura no espaço analisado. Na geometria, simetrias costumam ser apresentadas na forma de ações de grupos. A partir deste capítulo e até o final dessa dissertação faremos um estudo sobre ações que de alguma forma podem ter todas as suas informações traduzidas para algo mais simples com o auxílio da forma simplética. Esse capítulo é baseado em [1] com exemplos de [2].

\subsection{Ações Diferenciáveis}

Antes de começar, seria útil fazer uma revisão dos conceitos básicos de ações diferenciáveis para fixar a notação. Dado $G$ um grupo de Lie, denotaremos por $\operatorname{Lie}(G)$ a álgebra de Lie de $G$, ou então usaremos a letra gótica correspondente à letra usada para respresentar o grupo, no caso, $\mathfrak{g}$.

Vamos fixar algumas notações. Se $\psi: G \times M \rightarrow M$ é uma ação diferenciável, dados $g \in G$ e $p \in M$ denotaremos por $\psi_{g}: M \rightarrow M$ a aplicação tal que $\psi_{g}(p)=\psi(g, p)$. Analogamente, $\psi^{p}: G \rightarrow M$ será a aplicação dada por $\psi^{p}(g)=\psi(g, p)$. Observe que $\psi^{p}(G)=G(p)$ a órbita de $p$.

Se $G$ age em $M$ variedade diferenciável pela ação $\psi: G \times M \rightarrow M$, podemos associar cada elemento $\eta \in \mathfrak{g}$ a um campo definido por

$$
X^{\eta}(p)=\left.\frac{\mathrm{d}}{\mathrm{d} t} \psi(\exp (t \eta), p)\right|_{t=0}
$$

A função $\mathfrak{g} \ni \eta \mapsto X^{\eta} \in \mathfrak{X}(M)$ é chamada ação infinitesimal associada a $\psi$.

A operação de grupo e a estrutura suave de um grupo de Lie podem ser usadas para definir duas ações, uma na sua álgebra de Lie e outra em seu dual, ambas definidas a seguir

Definição 2.2.1 (Ação Adjunta e Coadjunta). Dado G um grupo de Lie com álgebra de Lie $\mathfrak{g}$, podemos definir sua ação adjunta $\mathrm{Ad}: G \times \mathfrak{g} \rightarrow \mathfrak{g}$ por

$$
\operatorname{Ad}(g) \eta=\frac{\mathrm{d}}{\mathrm{d} t}\left(g \exp (t \eta) g^{-1}\right)
$$

para todo $g \in G$ e $\eta \in \mathfrak{g}$.

Podemos também definir a ação coadjunta de $G, \operatorname{Ad}^{*}: G \times \mathfrak{g}^{*} \rightarrow \mathfrak{g}^{*}$, dada por

$$
\operatorname{Ad}^{*}(g) \xi=\xi \circ \operatorname{Ad}\left(g^{-1}\right)
$$

para todo $g \in G$ e $\xi \in \mathfrak{g}^{*}$. Outra forma de escrever a ação coadjunta é $\operatorname{Ad}^{*}(g)=\operatorname{Ad}\left(g^{-1}\right)^{*}$

O lema a seguir mostra o que acontece quando transladamos a ação infinitesimal usando a diferencial da ação original. Ele será usado, por exemplo, na seção 2.4 para demonstrar o teorema 
de Kirillov-Kostant-Sourieau.

Lema 2.2.2. Sejam $G$ grupo de Lie, $M$ variedade diferenciável e $\psi: G \times M \rightarrow M$ ação diferenciável. Então

1. $X^{k_{1} \eta_{1}+k_{2} \eta_{2}}=k_{1} X^{\eta_{1}}+k_{2} X^{\eta_{2}}$ para todos $\eta_{1}, \eta_{2} \in \mathfrak{g} e k_{1}, k_{2} \in \mathbb{R}$

2. $\mathrm{d} \psi_{g}\left(X^{\eta}(p)\right)=X^{\operatorname{Ad}(g) \eta}\left(\psi_{g}(p)\right)$ para todos $\eta \in \mathfrak{g}$ e $g \in G$

Demonstração. O item 1 vem do fato de que a ação infinitesimal é na verdade a diferencial da ação original e, portanto, é linear. De fato, temos que, dado $p \in M$

$$
\begin{aligned}
X^{k_{1} \eta_{1}+k_{2} \eta_{2}}(p) & =\left.\frac{\mathrm{d}}{\mathrm{d} t} \psi\left(\exp \left(t\left(k_{1} \eta_{1}+k_{2} \eta_{2}\right)\right), p\right)\right|_{t=0} \\
& =\mathrm{d} \psi_{(e, p)}\left(k_{1} \eta_{1}+k_{2} \eta_{2}, 0\right) \\
& =k_{1} \mathrm{~d} \psi_{(e, p)}\left(\eta_{1}, 0\right)+k_{2} \mathrm{~d} \psi_{(e, p)}\left(\eta_{2}, 0\right) \\
& =\left.k_{1} \frac{\mathrm{d}}{\mathrm{d} t} \psi\left(\exp \left(t \eta_{1}\right), p\right)\right|_{t=0}+\left.k_{2} \frac{\mathrm{d}}{\mathrm{d} t} \psi\left(\exp \left(t \eta_{2}\right), p\right)\right|_{t=0} \\
& =k_{1} X^{\eta_{1}}(p)+k_{2} X^{\eta_{2}}(p)
\end{aligned}
$$

Para verificar o item 2, observe que

$$
\begin{aligned}
\mathrm{d} \psi_{g}\left(X^{\eta}(p)\right) & =\left.\frac{\mathrm{d}}{\mathrm{d} t} \psi(g \exp (t \eta), p)\right|_{t=0} \\
& =\frac{\mathrm{d}}{\mathrm{d} t} \psi\left(g \exp (t \eta) g^{-1}, \psi(g, p)\right) \\
& =\frac{\mathrm{d}}{\mathrm{d} t} \psi(\exp (t \operatorname{Ad}(g)), \psi(g, p)) \\
& =X^{\operatorname{Ad}(g) \eta}\left(\psi_{g}(p)\right)
\end{aligned}
$$

A definição a seguir apresenta o conceito equivalente a morfismos para ações diferenciáveis, dado pelas chamadas funções equivariantes.

Definição 2.2.3. Sejam $\psi_{1}: G_{1} \times M_{1} \rightarrow M_{1}$ e $\psi_{2}: G_{2} \times M_{2} \rightarrow M_{2}$ ações diferenciáveis. Se $F: M_{1} \rightarrow M_{2}$ é uma função suave e $\varphi: G_{1} \rightarrow G_{2}$ é um homomorfismo de Lie, então o par $(F, \varphi)$ é dito equivariante com relação a $\psi_{1}$ e $\psi_{2}$ se vale que

$$
F\left(\psi_{1}(g, p)\right)=\psi_{2}(\varphi(g), F(p))
$$

Quando as ações estiverem consideradas claras, diremos apenas que o par $(F, \varphi)$ é equivariante.

Se $G_{1}=G_{2}=G$, diremos que $F$ é equivariante se $\left(F, \operatorname{Id}_{G}\right)$ for equivariante. Analogamente, se $M_{1}=M_{2}=M$, diremos que $\varphi$ é equivariante se $\left(\operatorname{Id}_{M}, \varphi\right)$ for equivariante.

\subsection{Ações Simpléticas, Hamiltonianas e Aplicação Momento}

Nesta seção tentaremos definir algumas propriedades que tornam uma ação útil em problemas de geometria simplética. A definição a seguir é a primeira e mais óbvia propriedade esperada de uma ação a ser usada com uma forma simplética.

Definição 2.3.1. Se G é um grupo de Lie e $(M, \omega)$ é uma variedade simplética, uma ação diferenciável $\psi: G \times M \rightarrow M$ é dita simplética se $\psi_{g}^{*} \omega=\omega$.

A proposição a seguir apresenta uma propriedade interessante de toda ação simplética. 
Proposição 2.3.2. Sejam $(M, \omega)$ variedade simplética, $G$ grupo de Lie com álgebra de Lie $\mathfrak{g}$ e $\psi$ : $G \times M \rightarrow M$ ação simplética. Então, os campos infinitesimais de $\psi$ são localmente Hamiltoneanos, ou seja, dados $\eta \in \mathfrak{g}$ e $p \in M$, existe $U$ aberto em $M$ contendo $p$ tal que $\left.X^{\eta}\right|_{U}$ é campo Hamiltoneano.

Demonstração. Observe primeiro que $\psi$ ser simplética é equivalente a dizer que a derivada de Lie de $\omega$ nas direções tangentes às órbitas de $\psi$ é zero. De fato, para todo $\eta \in \mathfrak{g}$ temos que

$$
\begin{aligned}
\mathcal{L}_{X \eta} \omega & =\lim _{t \rightarrow 0} \frac{\psi_{\exp (t \eta)}^{*} \omega-\omega}{t} \\
& =\lim _{t \rightarrow 0} \frac{\omega-\omega}{t} \\
& =0
\end{aligned}
$$

Isso, juntamente com a fórmula mágica de Cartan e o fato de $\omega$ ser fechada nos dá que

$$
\begin{aligned}
0 & =\mathcal{L}_{X \eta} \omega \\
& =\mathrm{d} \iota_{X} \eta \omega+\iota_{X} \eta \mathrm{d} \omega \\
& =\mathrm{d} \iota_{X} \eta \omega
\end{aligned}
$$

onde $\iota_{X} \eta$ representação a contração da forma por $X^{\eta}$.

Logo $\iota_{X} \eta \omega$ é forma fechada. Isso implica que, para todo $p \in M$ existe $U$ aberto contendo $p$ tal que $\left.\iota_{X} \eta \omega\right|_{U}$ é exata, ou seja, existe $H: U \rightarrow \mathbb{R}$ tal que $\mathrm{d} H=\left.\iota_{X}{ }^{\eta} \omega\right|_{U}$, ou equivalentemente, $\left.X^{\eta}\right|_{U}=X_{H}$, provando a proposição.

A proposição acima determina uma propriedade local de todas as ações simpléticas. Estaremos interessados daqui em diante em casos em que essa propriedade vale globalmente. Vamos começar estudando um caso particular.

Exemplo 2.3.3 (Ação $S^{1}$ ). Considere $\mathbb{C} \approx \mathbb{R}^{2}$ dotado da forma simplética $\omega=\mathrm{d} x \wedge \mathrm{d} y$ e a ação $\psi: S^{1} \times \mathbb{C} \rightarrow \mathbb{C}$ definida por

$$
\psi(g, z)=g z
$$

onde usamos a identificação $S^{1}=\{z \in \mathbb{C}|| z \mid=1\}$. Como a álgebra de Lie é unidimensional podemos identificá-la com $\mathbb{R}$ de forma que a exponencial de Lie pode ser escrita $\exp (\eta)=e^{2 \pi i \eta}$, para $\eta \in \mathbb{R}$. Dessa forma, o item 1 do lema 2.2.2 nos garante que todos os campos gerados pela ação infinitesimal de $\psi$ são múltiplos de $X^{1}$. Logo, basta encontrar um campo Hamiltoneano $H$ para $X^{1}$ e os teremos que $X^{c}$ terá campo Hamiltoneano $c H$. Vamos começar calculando o campo $X^{1}$.

$$
\begin{aligned}
X^{1}(z) & =\left.\frac{\mathrm{d}}{\mathrm{d} t} \psi(\exp (t), z)\right|_{t=0} \\
& =\left.\frac{\mathrm{d}}{\mathrm{d} t} e^{2 \pi i t} z\right|_{t=0} \\
& =2 \pi i z
\end{aligned}
$$

Logo queremos encontrar uma função $H: \mathbb{C} \rightarrow \mathbb{R}$ tal que

$$
\begin{aligned}
\mathrm{d} H_{z}(w) & =\omega\left(X^{1}(z), w\right) \\
& =\mathrm{d} x \wedge \mathrm{d} y(2 \pi i z, w) \\
& =2 \pi(\operatorname{Re}(i z) \operatorname{Im}(w)-\operatorname{Im}(i z) \operatorname{Re}(w)) \\
& =-2 \pi(\operatorname{Im}(z) \operatorname{Im}(w)+\operatorname{Re}(z) \operatorname{Re}(w))
\end{aligned}
$$

Logo a matriz da diferencial de $H$ é dada por

$$
\left[\mathrm{d} H_{z}\right]=\left[\begin{array}{ll}
-2 \pi \operatorname{Re}(z) & -2 \pi \operatorname{Im}(z)
\end{array}\right]
$$


e temos que $H$ é a função

$$
\begin{aligned}
H(z) & =-\pi\left(\operatorname{Re}(z)^{2}+\operatorname{Im}(z)^{2}\right)+k \\
& =-\pi|z|^{2}+k
\end{aligned}
$$

onde $k \in \mathbb{R}$ é uma constante.

Logo, como já foi observado acima, cada campo infinitesimal $X^{c}$ é gradiente simplético da função $H_{c}(z):=-c \pi|z|^{2}+k$. Podemos então definir uma função $\mu^{*}: \mathbb{R} \rightarrow C^{\infty}(\mathbb{C})$, tal que $\mu^{*}(c)=H_{c}$, que contém toda a informação da ação, traduzida via forma simplética. A aplicação $\mu^{*}$ é chamada aplicação comomento.

Vamos agora generalizar o exemplo acima para o caso em que $G$ é um grupo de Lie conexo qualquer. Seria natural definir $\mu^{*}$ tal que $\mu^{*}(\eta)$ seja Hamiltoneano do campo $X^{\eta}$ para todo $\eta \in$ g. Ações dessa forma são chamadas fracamente Hamiltoneanas. A definição formal de aplicação comomento é a seguinte.

Definição 2.3.4 (Aplicação Comomento). Dada uma ação diferenciável $\psi: G \times M \rightarrow M$ em uma variedade simplética, a aplicação comomento para $\psi$ é uma aplicação linear $\mu^{*}: \mathfrak{g} \rightarrow C^{\infty}(M)$ tal que:

1. $X_{\mu^{*}(\eta)}=X^{\eta}, \forall \eta \in \mathfrak{g}$

2. $\mu^{*}([X, Y])=\left\{\mu^{*}(X), \mu^{*}(Y)\right\}$, onde $\{f, g\}=\omega\left(X_{f}, X_{g}\right)$ é o colchete de Poisson.

Se $\psi$ admite aplicação comomento então a ação é dita Hamiltoneana.

Em palavras, $\mu^{*}$ associa a cada elemento $\eta \in \mathfrak{g}$ uma função Hamiltoneana para o campo $X_{\eta} \mathrm{e}$ é um homomorfismo entre álgebras de Lie.

Note que no caso $G=S^{1}$ a segunda condição é sempre satsifeita. De fato, $\mu^{*}$ ser linear e a álgebra de Lie $\mathfrak{g}$ de $S^{1}$ ser unidimensional implica que $\mu^{*}(X)$ e $\mu^{*}(Y)$ são múltiplos. Logo $\left\{\mu^{*}(X), \mu^{*}(Y)\right\}=$ 0 , pois o colchete de Poisson é alternado. Por outro lado, $\mathfrak{g}$ admite apenas o colchete nulo, e $\mu^{*}([X, Y])=0$.

Podemos ver a aplicação comomento como uma função de dois argumentos, associando um elemento da álgebra de Lie e um ponto de $M$ a um número real. Em muitas situações será mais interessante inverter a ordem em que os argumentos são passados, associando a cada elemento de $M$ um funcional linear em $\mathfrak{g}^{*}$. A função que obtemos fazendo essa inversão é chamada aplicação momento.

Definição 2.3.5 (Aplicação Momento). Dada uma ação Hamiltoneana $\psi: G \times M \rightarrow M$ em uma variedade simplética dotada de aplicação comomento $\mu^{*}$, a aplicação momento para $\psi$ é uma aplicação $\mu: M \rightarrow \mathfrak{g}^{*}$ satisfazendo $\mu(p)(\eta)=\mu^{*}(\eta)(p)$, para todos $p \in M$ e $\eta \in \mathfrak{g}$. Daqui em diante, denotaremos $\mu^{\eta}=\mu^{*}(\eta)$, ou seja $\mu^{\eta}: M \rightarrow \mathbb{R}$ é a aplicação dada por $\mu^{\eta}(p)=\mu(p)(\eta)$

A proposição a seguir traduz as propriedades que definem a aplicação comomento em termos da aplicação momento. A prova foi retirada de [5]

Antes disso, apresentaremos um lema técnico sem sua prova.

Lema 2.3.6. Seja $G$ grupo de Lie conexo com álgebra de Lie $\mathfrak{g}$. Para todos $\eta, \tilde{\eta} \in \mathfrak{g}$

1. $\operatorname{Ad}_{\exp (-t \eta)} \eta=\eta$

2. $\frac{\mathrm{d}}{\mathrm{d} t} \operatorname{Ad}_{\exp (-t \tilde{\eta})} \eta=\left[\operatorname{Ad}_{\exp (-t \tilde{\eta})}, \tilde{\eta}\right]$

Proposição 2.3.7. Sejam $(M, \omega)$ variedade simplética, $G$ grupo de Lie conexo com álgebra de Lie g. Se $\psi: G \times M \rightarrow M$ é ação Hamiltoneana, temos que $\mu: M \rightarrow \mathfrak{g}^{*}$ é aplicação momento para $\psi$ se, e só se, vale que 
1. $X_{\mu^{\eta}}=X^{\eta}, \forall \eta \in \mathfrak{g}$

2. $\mu$ é equivariante, i.e, $\mu \circ \psi_{g}=\operatorname{Ad}_{g}^{*} \circ \mu$

Demonstração. O item 1 é óbvio da definição das aplicações momento e comomento.

Para provar o item 2 , observe que, se $\mu^{*}$ for aplicação comomenti para $\psi$, é quivalente provar que, para todos $g \in G$ e $\eta \in \mathfrak{g}$, vale que $\mu^{*}(\eta) \circ \psi_{g}=\operatorname{Ad}_{g}^{*} \circ \mu^{*}(\eta)$. Como $G$ é conexo, todo elemento $g \in G$ pode ser escrito como $\exp (\tilde{\eta})$, para algum $\tilde{\eta} \in \mathfrak{g}$. Dessa forma, $\operatorname{como} \mu^{*}(\eta) \circ \psi_{e}=\mu^{*}(\eta)=\operatorname{Ad}_{e}^{*} \circ \mu^{*}(\eta)$, basta provar a igualdade $\frac{\mathrm{d}}{\mathrm{d} t} \mu^{*}(\eta) \circ \psi_{\exp (t \eta)}=\frac{\mathrm{d}}{\mathrm{d} t} \operatorname{Ad}_{\exp (t \eta)}^{*} \circ \mu^{*}(\eta)$ para todo $\eta \in \mathfrak{g}$. Observe então que, dados $p \in M$ e $\eta, \tilde{\eta} \in \mathfrak{g}^{*}$

$$
\begin{aligned}
\frac{\mathrm{d}}{\mathrm{d} t} \mu^{*} \circ \psi_{\exp (t \tilde{\eta})}(\eta)(p) & =\mathrm{d}\left(\mu^{*}(\eta)\right)_{\psi_{\exp (t \tilde{\eta})}(p)}\left(\frac{\mathrm{d}}{\mathrm{d} t} \psi_{\exp (t \tilde{\eta})}(p)\right) \\
& =\omega\left(X_{\mu^{*}(\eta)}\left(\psi_{\exp (t \tilde{\eta})}(p)\right),\left.\frac{\mathrm{d}}{\mathrm{d} s} \psi_{\exp (s \tilde{\eta})}\left(\psi_{\exp (t \tilde{\eta})}(p)\right)\right|_{s=0}\right) \\
& =\omega\left(X^{\eta}\left(\psi_{\exp (t \tilde{\eta})}(p)\right), X^{\tilde{\eta}}\left(\psi_{\exp (t \tilde{\eta})}(p)\right)\right) \\
& =\omega\left(\mathrm{d} \psi_{\exp (-\mathrm{t} \tilde{\eta})} \circ X^{\eta}\left(\psi_{\exp (t \tilde{\eta})}(p)\right), \mathrm{d} \psi_{\exp (-\mathrm{t} \tilde{\eta})} \circ X^{\tilde{\eta}}\left(\psi_{\exp (t \tilde{\eta})}(p)\right)\right) \\
& =\omega\left(X^{\operatorname{Ad} \exp (-\mathrm{t} \tilde{\eta})^{\eta}\left(\psi_{\exp (-\mathrm{t} \tilde{\eta})} \circ \psi_{\exp (t \tilde{\eta})}(p)\right), X^{\operatorname{Ad}} \exp (-\mathrm{t} \tilde{\eta}) \tilde{\eta}}\left(\psi_{\exp (-\mathrm{t} \tilde{\eta})} \circ \psi_{\exp (t \tilde{\eta})}(p)\right)\right) \\
& =\omega\left(X^{\operatorname{Ad}_{\exp (-\mathrm{t} \tilde{\eta})} \eta}(p), X^{\tilde{\eta}}(p)\right) \\
& =\omega\left(X_{\mu^{*}\left(\operatorname{Ad}_{\exp (-\mathrm{t} \tilde{\eta})}\right)}(p), X_{\mu^{*}(\tilde{\eta})}(p)\right)
\end{aligned}
$$

Por outro lado, observe que

$$
\begin{aligned}
\frac{\mathrm{d}}{\mathrm{d} t} \operatorname{Ad}_{\exp (t \tilde{\eta})}^{*} \circ \mu^{*}(\eta)(p) & =\frac{\mathrm{d}}{\mathrm{d} t} \mu^{*}\left(\operatorname{Ad}_{\exp (-t \tilde{\eta})} \eta\right)(p) \\
& =\mu^{*}\left(\frac{\mathrm{d}}{\mathrm{d} t} \operatorname{Ad}_{\exp (-t \tilde{\eta})} \eta\right)(p) \\
& =\mu^{*}\left(\left[\operatorname{Ad}_{\exp (-\mathrm{t} \tilde{\eta})} \eta, \tilde{\eta}\right]\right)(p) \\
& =\left\{\mu^{*}\left(\operatorname{Ad}_{\exp (-\mathrm{t} \tilde{\eta})} \eta\right), \mu^{*}(\tilde{\eta})\right\}(p) \\
& =\omega\left(X_{\mu^{*}\left(\operatorname{Ad}_{\exp (-\mathrm{t} \tilde{\eta})} \eta\right)}(p), X_{\mu^{*}(\tilde{\eta})}(p)\right)
\end{aligned}
$$

Vamos agora introduzir um lema que nos permite criar novas aplicações momento a partir de ações Hamiltoneanas conhecidas.

Lema 2.3.8. Sejam $\left(M_{1}, \omega_{1}\right)$ e $\left(M_{2}, \omega_{2}\right)$ variedades simpléticas, $G_{1}$ e $G_{2}$ grupos de Lie com álgebras de Lie $\mathfrak{g}_{1}$ e $\mathfrak{g}_{2}$. Se $\psi_{1}: G_{1} \times M_{1} \rightarrow M_{1}$ e $\psi_{2}: G_{2} \times M_{2} \rightarrow M_{2}$ são ações Hamiltoneanas com aplicação momento $\mu_{1}$ e $\mu_{2}$, então a ação $\psi:\left(G_{1} \times G_{2}\right) \times\left(M_{1} \times M_{2}\right) \rightarrow M_{1} \times M_{2}$ dada por

$$
\psi\left(\left(g_{1}, g_{2}\right),\left(p_{1}, p_{2}\right)\right)=\left(\psi_{1}\left(g_{1}, p_{1}\right), \psi_{2}\left(g_{2}, p_{2}\right)\right)
$$

é Hamiltoneana com aplicação momento $\mu: M_{1} \times M_{2} \rightarrow\left(\mathfrak{g}_{1} \oplus \mathfrak{g}_{2}\right)^{*}$ dada por

$$
\mu\left(p_{1}, p_{2}\right)\left(\eta^{1}, \eta^{2}\right)=\mu_{1}\left(p_{1}\right)\left(\eta^{1}\right)+\mu_{2}\left(p_{2}\right)\left(\eta^{2}\right)
$$

Demonstração. Sejam $p_{1} \in M_{1}, p_{2} \in M_{2}, v_{1} \in T_{p_{1}} M_{1}, v_{2} \in T_{p_{2}} M_{2}, \eta^{1} \in \mathfrak{g}_{1}$ e $\eta^{2} \in \mathfrak{g}_{2}$. Então usando 
a forma simplética produto $\omega$ definida em 1.2.6, temos que,

$$
\begin{aligned}
\omega\left(X_{\mu^{\left(\eta^{1}, \eta^{2}\right)}}\left(p_{1}, p_{2}\right),\left(v_{1}, v_{2}\right)\right) & =\mathrm{d} \mu^{\left(\eta^{1}, \eta^{2}\right)}\left(v_{1}, v_{2}\right) \\
& =\mathrm{d} \mu_{1}^{\eta^{1}}\left(v_{1}\right)+\mathrm{d} \mu_{2}^{\eta^{2}}\left(v_{2}\right) \\
& =\omega_{1}\left(X_{\mu_{1}^{\eta^{1}}}\left(p_{1}\right), v_{1}\right)+\omega_{2}\left(X_{\mu_{2}^{\eta^{2}}}\left(p_{2}\right), v_{2}\right) \\
& =\omega_{1}\left(X^{\eta^{1}}\left(p_{1}\right), v_{1}\right)+\omega_{2}\left(X^{\eta^{2}}\left(p_{2}\right), v_{2}\right) \\
& =\omega\left(X^{\left(\eta^{1}, \eta^{2}\right)}\left(p_{1}, p_{2}\right),\left(v_{1}, v_{2}\right)\right)
\end{aligned}
$$

O exemplo a seguir ilustra o uso o lema 2.3.8. Além disso, o par ação Hamiltoneana e sua aplicação momento apresentado será usado na seção 3.4 para dar uma idéia da prova do teorema de Delzant

Exemplo 2.3.9 (Ação do toro em $\mathbb{C}^{n}$ ). Vamos agora aplicar o lema 2.3.8 à ação apresentada no exemplo 2.3.3. Seja $\varphi: S^{1} \times \mathbb{C} \rightarrow \mathbb{C}$ a ação do exemplo 2.3.3.Defina a ação $\psi: T^{n} \times \mathbb{C}^{n} \rightarrow \mathbb{C}^{n}$ dada por

$$
\left(T^{n}, \mathbb{C}^{n}\right) \ni\left(\left(g_{1}, \ldots, g_{n}\right),\left(z_{1}, \ldots, z_{n}\right)\right) \mapsto\left(g_{1} z_{1}, \ldots, g_{n} z_{n}\right)
$$

usando as identificações $T^{n}=S^{1} \times \ldots \times S^{1}$ e $S^{1}=\{z \in \mathbb{C}|| z \mid=1\}$.

O lema 2.3.8 e os resultados do exemplo 2.3.3 nos garante então que $\psi$ é uma ação Hamiltoneana com aplicação momento $\mu: \mathbb{C}^{n} \rightarrow\left(\mathbb{R}^{n}\right)^{*}$ dada por

$$
\mu\left(z_{1}, \ldots, z_{n}\right)\left(t_{1}, \ldots, t_{n}\right)=\pi\left(\left(\left|z_{1}\right|^{2}+k_{1}\right) t_{1}+\ldots+\left(\left|z_{n}\right|^{2}+k_{n}\right) t_{n}\right)
$$

onde $k_{1}, \ldots, k_{n} \in \mathbb{R}$ são constantes.

A aplicação momento foi assim nomeada por, em casos particulares, coincidir com grandezas físicas de mesmo nome. Os dois exemplos a seguir ilustram casos em que a aplicação momento coincide com o momento linear e momento angular, respectivamente.

Exemplo 2.3.10 (Momento linear). Considere $\mathrm{TR}^{3} \approx \mathbb{R}^{6}$ munido da sua forma simplética canônica $\omega$. Usando a identificação $\mathbb{R}^{6} \approx \mathbb{R}^{3} \times \mathbb{R}^{3}$, considere a ação $\psi: \mathbb{R}^{3} \times \mathbb{R}^{6} \rightarrow \mathbb{R}^{6}$ definida por $\psi(g,(q, p))=(q+g, p)$. Vamos calcular a ação infinitesimal induzida por $\psi$. Dados $(q, p) \in \mathbb{R}^{6} \mathrm{e}$ $\eta \in \operatorname{Lie}\left(\mathbb{R}^{3}\right)=\mathbb{R}^{3}$

$$
\begin{aligned}
X^{\eta}(q, p) & =\left.\frac{\mathrm{d}}{\mathrm{d} t} \psi^{(q, p)}(\exp (t \eta))\right|_{t=0} \\
& =\left.\frac{\mathrm{d}}{\mathrm{d} t} \psi^{(q, p)}(t \eta)\right|_{t=0} \\
& =\left.\frac{\mathrm{d}}{\mathrm{d} t}(q+t \eta, p)\right|_{t=0} \\
& =(\eta, 0)
\end{aligned}
$$

Logo, se $\mu: \mathbb{R}^{6} \rightarrow\left(\mathbb{R}^{3}\right)^{*}$ é um candidato a aplicação momento, precisamos que, para todos $\eta \in \mathbb{R}^{3}$ e $(q, p),\left(v_{1}, v_{2}\right) \in \mathbb{R}^{6}$

$$
\begin{aligned}
\mathrm{d}\left(\mu^{\eta}\right)_{(q, p)}\left(v_{1}, v_{2}\right) & =\omega\left(X^{\eta}(q, p),\left(v_{1}, v_{2}\right)\right) \\
& =\omega\left((\eta, 0),\left(v_{1}, v_{2}\right)\right) \\
& =\left\langle\eta, v_{2}\right\rangle
\end{aligned}
$$


Substituindo $\left(v_{1}, v_{2}\right)$ por elementos da base canônica de $\mathbb{R}^{6}$, obtemos as equações

$$
\begin{aligned}
& \frac{\partial \mu^{\eta}}{\partial q_{i}}(q, p)=0 \\
& \frac{\partial \mu^{\eta}}{\partial p_{i}}(q, p)=\eta_{i}
\end{aligned}
$$

Logo, uma solução é $\mu^{\eta}(q, p)=\langle p, \eta\rangle$ e $\mu(q, p)=\langle p, \cdot\rangle$. Logo, a menos da identificação de Riesz, $\mu$ corresponde ao momento linear.

Para ver que $\mu$ é realmente aplicação momento para $\psi$ precisamos verificar a condição de equivariância. Observe, no entanto, que $\mathbb{R}^{3}$ é um grupo abeliano, logo $\operatorname{Ad}_{g}^{*}=\operatorname{Id}_{\left(\mathbb{R}^{3}\right)^{*}}$, para todo $g \in \mathbb{R}^{3}$. Logo temos que

$$
\begin{aligned}
\mu \circ \psi_{g}(q, p) & =\mu(q+g, p) \\
& =\langle p, \cdot\rangle \\
& =\mu(q, p) \\
& =\operatorname{Ad}_{g}^{*} \circ \mu(q, p)
\end{aligned}
$$

O exemplo do momento angular requer um lema sobre cálculo matricial. Esse lema será usado apenas na prova do exemplo 2.3.12 e o leitor que deseje pode pular sua demonstração sem perdas no restante dessa dissertação.

Lema 2.3.11. Seja $f: \mathbb{R}^{3} \rightarrow \mathfrak{s o}(3)$ definido por

$$
\left(\begin{array}{l}
\eta_{1} \\
\eta_{2} \\
\eta_{3}
\end{array}\right) \mapsto\left[\begin{array}{ccc}
0 & -\eta_{3} & \eta_{2} \\
\eta_{3} & 0 & -\eta_{1} \\
-\eta_{2} & \eta_{1} & 0
\end{array}\right]
$$

e defina $F: \operatorname{so}(3)^{*} \rightarrow \mathbb{R}^{3}$ como o pull-back por $f$ composto com a identificação $\mathbb{R}^{3} \approx\left(\mathbb{R}^{3}\right)^{*}$. Então $f$ e $F$ são isomorfismos e vale que, para todos $v, w \in \mathbb{R}^{3}, g \in \mathrm{SO}(3)$ e $\xi \in \operatorname{so}(3)^{*}$

1. $f(v) w=v \times w$

2. $f((g \cdot v) \times(g \cdot w))=\operatorname{Ad}_{g} f(v \times w)$

3. $\xi(f(v))=\langle F(\xi), v\rangle$

Demonstração. O item 1 pode ser verificado apenas calculando os dois lados a equação e não será provado aqui.

Para provar o item 2 note primeiro que, se as matrizes linha $g_{1}, g_{2}, g_{3}$ são as linhas de $g$, temos

$$
(g \cdot v) \times(g \cdot w)=\left(\begin{array}{l}
g_{2} v g_{3} w-g_{2} w g_{3} v \\
g_{3} v g_{1} w-g_{3} w g_{1} v \\
g_{1} v g_{2} w-g_{1} w g_{2} v
\end{array}\right)
$$

Temos também que

$$
g_{i} v g_{j} w-g_{i} w g_{j} v=g_{i}\left(v w^{T}-w v^{T}\right) g_{j}^{T}
$$

para $i, j \in\{1,2,3\}$. Para simplificar a notação, defina $V:=v w^{T}-w v^{T}$. Podemos reescrever 2.1 como

$$
(g \cdot v) \times(g \cdot w)=\left(\begin{array}{c}
g_{2} V g_{3}^{T} \\
g_{3} V g_{1}^{T} \\
g_{1} V g_{2}^{T}
\end{array}\right)
$$


Observe que a matriz

$$
V=\left[\begin{array}{ccc}
0 & -\left(v_{1} w_{2}-v_{2} w_{1}\right) & v_{3} w_{1}-v_{1} w_{3} \\
v_{1} w_{2}-v_{2} w_{1} & 0 & -\left(v_{2} w_{3}-v_{3} w_{2}\right) \\
-\left(v_{3} w_{1}-v_{1} w_{3}\right) & v_{2} w_{3}-v_{3} w_{2} & 0
\end{array}\right]=-f(v \times w)
$$

Veja também que, para $i \in\{1,2,3\}$ temos $g_{i} v g_{i} w=g_{i} w g_{i} v$, já que $g_{i} v$ e $g_{i} w$ são números reais, $\operatorname{logo} g_{i} V g_{i}^{T}=0$. Isso nos dá que

$$
\begin{aligned}
\operatorname{Ad}_{g} f(v \times w) & =g \cdot(-V) \cdot g^{-1} \\
& =g \cdot(-V) \cdot g^{T} \\
& =\left[\begin{array}{ccc}
0 & -g_{1} V g_{2}^{T} & -g_{1} V g_{3}^{T} \\
-g_{2} V g_{1}^{T} & 0 & -g_{2} V g_{3}^{T} \\
-g_{3} V g_{1}^{T} & -g_{3} V g_{2}^{T} & 0
\end{array}\right] \\
& =\left[\begin{array}{ccc}
0 & -g_{1} V g_{2}^{T} & g_{3} V g_{1}^{T} \\
g_{1} V g_{2}^{T} & 0 & -g_{1} V g_{3}^{T} \\
-g_{3} V g_{1}^{T} & g_{2} V g_{3}^{T} & 0
\end{array}\right]
\end{aligned}
$$

onde a última igualdade vem da antissimetria de $V$ e do fato de que $g_{i} V g_{j}^{T}=\left(g_{i} V g_{j}^{T}\right)^{T}$ por ser uma matriz $1 \times 1$.

Logo, 2.2 e 2.3 nos dão

$\operatorname{Ad}_{g} f(v \times w)=\left[\begin{array}{ccc}0 & -((g \cdot v) \times(g \cdot w))_{3} & ((g \cdot v) \times(g \cdot w))_{2} \\ ((g \cdot v) \times(g \cdot w))_{3} & 0 & -((g \cdot v) \times(g \cdot w))_{1} \\ -((g \cdot v) \times(g \cdot w))_{2} & ((g \cdot v) \times(g \cdot w))_{1} & 0\end{array}\right]=f((g \cdot v) \times(g \cdot w))$

Exemplo 2.3.12 (Momento angular). Considere $\psi: \mathrm{SO}(3) \times \mathrm{T}^{*} \mathbb{R}^{3}$ a ação dada por $\psi_{g}(q, p)=$ $(g q, g p)$, onde $g \in \mathrm{SO}(3),(q, p) \in \mathrm{T}^{*} \mathbb{R}^{3} \approx \mathbb{R}^{6}$ e $g q$ representa a ação usual de $\mathrm{SO}(3)$ em $\mathbb{R}^{3}$.

Se $\psi$ admite aplicação momento $\mu: \mathrm{T}^{*} \mathbb{R}^{3} \rightarrow \mathfrak{s o}(3)^{*}$, é necessário que

$$
X_{\mu^{\eta}}=X^{\eta}
$$

para todo $\eta \in \operatorname{so}(3)$.

Vamos calcular explicitamente ambos os termos da equação. Note que como a função $f$ definida no lema 2.3.11 é um isomorfismo, sempre podemos escrever $\eta$ unicamente como $f(v)$, para algum $v \in \mathbb{R}^{3}$. Assim, se $(q, p) \in \mathbb{R}^{6}$, temos que

$$
\begin{aligned}
X^{f(v)}(v) & =\left.\frac{\mathrm{d}}{\mathrm{d} t} \psi_{\exp (t f(v))}(q, p)\right|_{t=0} \\
& =\left.\frac{\mathrm{d}}{\mathrm{d} t}(\exp (t f(v)) q, \exp (t f(v)) p)\right|_{t=0} \\
& =(f(v) q, f(v) p) \\
& =(v \times q, v \times p)
\end{aligned}
$$

Para calcular $X_{\mu^{f(v)}}$, note que a observação 1.2.5 nos dá que a forma simplética cotangente de $\mathrm{T}^{*} \mathbb{R}^{3}$ coincide com a forma simpétlica canônica de $\mathbb{R}^{6}$. Logo, se escrevermos $X_{\mu^{f(v)}}=\left(X_{\mu^{f(v)}}^{1}, X_{\mu^{f(v)}}^{2}\right) \in$ $\mathbb{R}^{3} \times \mathbb{R}^{3}$, temos que, para todos $\left(v_{1}, v_{2}\right),\left(w_{1}, w_{2}\right) \in \mathbb{R}^{3} \times \mathbb{R}^{3}$.

$$
\begin{aligned}
\mathrm{d} \mu_{\left(v_{1}, v_{2}\right)}^{f(v)}\left(w_{1}, w_{2}\right) & =\omega\left(X_{\mu^{f(v)}}\left(v_{1}, v_{2}\right),\left(w_{1}, w_{2}\right)\right) \\
& =\left\langle X_{\mu^{f(v)}}^{1}\left(v_{1}, v_{2}\right), w_{2}\right\rangle-\left\langle X_{\mu^{f(v)}}^{2}\left(v_{1}, v_{2}\right), w_{1}\right\rangle
\end{aligned}
$$


Fazendo $w_{1}=0$, temos que

$$
X_{\mu^{f(v)}}^{1}\left(v_{1}, v_{2}\right)=\frac{\partial \mu^{f(v)}}{\partial p}\left(v_{1}, v_{2}\right)
$$

Analogamente, fazendo $w_{2}=0$ obtemos

$$
X_{\mu f(v)}^{2}\left(v_{1}, v_{2}\right)=-\frac{\partial \mu^{f(v)}}{\partial q}\left(v_{1}, v_{2}\right)
$$

Logo

$$
X_{\mu^{f(v)}}=\left(\frac{\partial \mu^{f(v)}}{\partial p},-\frac{\partial \mu^{f(v)}}{\partial q}\right)
$$

Temos então, que a condição 2.4 é equivalente a

$$
\left(\frac{\partial \mu^{f(v)}}{\partial p},-\frac{\partial \mu^{f(v)}}{\partial q}\right)=(v \times q, v \times p)
$$

A função $\mu^{f(v)}(q, p)=\langle(q \times p), v\rangle$ satisfaz essa condição. De fato, a propriedade de alternância do produto misto garante que $\mu^{f(v)}(q, p)=\langle(v \times q), p\rangle$, tornando a equação

$$
\frac{\partial \mu^{f(v)}}{\partial p}=v \times q
$$

trivial. Por outro lado, a mesa propriedade nos dá $\mu^{f(v)}(q, p)=-\langle(v \times p), q\rangle$, e, portanto,

$$
\frac{\partial \mu^{f(v)}}{\partial q}=-v \times p
$$

Assim, temos, usando $F$ como no lema 2.3.11

$$
\begin{aligned}
\langle(q \times p), v\rangle & =\mu^{f(v)}(q, p) \\
& =\mu(q, p)(f(v)) \\
& =\langle F \circ \mu(q, p), v\rangle
\end{aligned}
$$

para todo $v \in \mathbb{R}^{3}$. Isso nos dá que, $\mu(q, p)$ é o elemento de $\mathfrak{s o}(3)$ que é identificado por $F \operatorname{com} q \times p$ o momento angular.

Para confirmar que $\mu$ é momento nos resta mostrar que $\mu \circ \psi_{g}=A d_{g}^{*} \circ \mu$. Dados, $(q, p) \in \mathbb{R}^{6}$, $v \in \mathbb{R}^{3}$ e $A \in \mathrm{SO}(3)$, temos que

$$
\left(\operatorname{Ad}_{A}^{*} \circ \mu\right)^{f(v)}(q, p)=\mu^{\operatorname{Ad}_{A^{-1}} f(v)}(q, p)
$$

Seja $w_{A} \in \mathbb{R}^{3}$ tal que $f\left(w_{A}\right)=\operatorname{Ad}_{A^{-1}} f(v)$. Note que podemos escolher a métrica $\langle\cdot, \cdot\rangle$ tal que 
a métrica $g=\left\langle f^{-1}(\cdot), f^{-1}(\cdot)\right\rangle$ em $\mathrm{SO}(3)$ é bi-invariante. Temos então que

$$
\begin{aligned}
\left(\operatorname{Ad}_{A}^{*} \circ \mu\right)^{f(v)}(q, p) & =\mu^{f\left(w_{A}\right)}(q, p) \\
& =\left\langle q \times p, w_{A}\right\rangle \\
& =g\left(f(q \times p), f\left(w_{A}\right)\right) \\
& =g\left(f(q \times p), \operatorname{Ad}_{A^{-1}} f(v)\right) \\
& =g\left(\operatorname{Ad}_{A} f(q \times p), f(v)\right) \\
& =g(f(A q \times A p), f(v)) \\
& =\langle A q \times A p, v\rangle \\
& =\mu^{f(v)}(A q, A p) \\
& =\left(\mu \circ \psi_{A}\right)^{f(v)}(q, p)
\end{aligned}
$$

A seguir apresentaremos dois casos particulares de ações Hamiltoneanas. Veremos que esses exemplos generalizam alguns dos casos já apresentados. Não será feita a prova dos resultados em si, apenas que eles são casos gerais de exemplos já apresentados. A demonstração de cada um pode ser encontrada em [2].

Exemplo 2.3.13 (Ação linear simplética). Seja $(V, \omega)$ uma espaço vetorial simplético e $G$ um grupo de Lie com álgebra de Lie $\mathfrak{g}$. Se $\psi: G \times V \rightarrow V$ é uma ação simplética em $V$, então $\psi$ é Hamiltoneana com aplicação momento $\mu: V \rightarrow \mathfrak{g}^{*}$ dada por $\mu^{\eta}(v)=\frac{1}{2} \omega\left(X^{\eta}(v), v\right)$ Observe que esse resultado generaliza o exemplo 2.3.12. De fato, dada $\psi^{\text {ang }}: \mathrm{SO}(3) \times \mathbb{R}^{6} \rightarrow \mathbb{R}^{6}$, ação do exemplo citado, ou seja, tal que $\psi_{g}^{a n g}(q, p)=(g q, g p)$. $\psi^{a n g}$ é claramente linear e, portanto, $\mathrm{d}\left(\psi_{g}^{a n g}\right)_{q, p}=\psi_{g}^{a n g}$. Assim, se $\omega$ é a forma simplética canônica de $\mathbb{R}^{6}$, dados $\left(q_{1}, p_{1}\right),\left(q_{2}, p_{2}\right) \in \mathbb{R}^{6}$, temos

$$
\begin{aligned}
\left(\psi_{g}^{\text {ang }}\right)^{*} \omega\left(\left(q_{1}, p_{1}\right),\left(q_{2}, p_{2}\right)\right)=\omega\left(\left(\mathrm{d} \psi_{g}^{a n g}\right)\left(q_{1}, p_{1}\right),\left(\mathrm{d} \psi_{g}^{a n g}\right)\left(q_{2}, p_{2}\right)\right) & \\
& =\omega\left(\left(g q_{1}, g p_{1}\right),\left(g q_{2}, g p_{2}\right)\right) \\
& =\left\langle g q_{1}, g p_{2}\right\rangle-\left\langle g p_{1}, g q_{2}\right\rangle \\
& =\left\langle q_{1}, p_{2}\right\rangle-\left\langle p_{1}, q_{2}\right\rangle \\
& =\omega\left(\left(q_{1}, p_{1}\right),\left(q_{2}, p_{2}\right)\right)
\end{aligned}
$$

onde a quarta igualdade vem do fato que $g \in \mathrm{SO}(3)$. Logo, $\psi^{a n g}$ é simplética. Podemos então aplicar a fórmula para o momento dada acima. Dados $\eta=f(v) \in \operatorname{Lie}\left(\mathbb{R}^{3}\right)=\mathbb{R}^{3}$, onde $f$ é a função definida no lema 2.3.11, e $(q, p) \in \mathbb{R}^{6}$, temos que

$$
\begin{aligned}
\mu^{\eta}(q, p) & =\frac{1}{2} \omega\left(X^{f(v)}(q, p),(q, p)\right) \\
& =\frac{1}{2} \omega\left(\left.\frac{\mathrm{d}}{\mathrm{d} t} \psi^{a n g}(\exp (t f(v)),(q, p))\right|_{t=0},(q, p)\right) \\
& =\frac{1}{2} \omega\left(\left.\frac{\mathrm{d}}{\mathrm{d} t}\left(e^{t f(v)} q, e^{t f(v)} p\right)\right|_{t=0},(q, p)\right) \\
& =\frac{1}{2} \omega((v \times q, v \times p),(q, p)) \\
& =\frac{1}{2}(\langle v \times q, p\rangle-\langle v \times p, q\rangle) \\
& =\langle q \times p, v\rangle
\end{aligned}
$$

o que corresponde ao resultado encontrado no exemplo 2.3.12.

Exemplo 2.3.14 (Levantamento cotangente). Seja $M$ variedade diferenciável e $G$ grupo de Lie com álgebran de Lie $\mathfrak{g}$. Dada $\psi: G \times M \rightarrow M$ uma ação diferenciável, defina seu levantamento cotangente $\Psi: G \times \mathrm{T}^{*} M \rightarrow \mathrm{T}^{*} M$ por $\Psi_{g}=\mathrm{d} \psi_{g^{-1}}^{*}$. Então $\Psi$ é ação Hamiltoneana com relação à forma simplética canônica de $\mathrm{T}^{*} M$ com aplicação momento $\mu: \mathrm{T}^{*} M \rightarrow \mathfrak{g}^{*}$ dada pela fórmula $\mu^{\eta}\left(\alpha_{p}\right)=\alpha_{p}\left(X^{\eta}(p)\right)$, onde a ação infinitesimal considerada é a de $\psi$. 
Note que os exemplos 2.3.10 e 2.3.12 são casos particulares deste exemplo. De fato, considere a ação $\psi^{\text {lin }}: \mathbb{R}^{3} \times \mathbb{R}^{3} \rightarrow \mathbb{R}^{3}$ dada por $\psi^{\text {lin }}(g, q)=q+g$. Se $\Psi^{\text {lin }}$ é o levantamento cotagente de $\psi^{\text {lin }}$, temos $\Psi_{g}^{l i n}(q, p)=(q+g, p)$ que coincide com a ação apresentada no exemplo 2.3.10.

Vamos então calcular a aplicação momento de $\Psi^{\text {lin }}$ pelo método apresentado acima. Sejam $q \in \mathbb{R}^{3}, \eta \in \operatorname{Lie}\left(\mathbb{R}^{3}\right)=\mathbb{R}^{3}$ e $\alpha_{q}=\langle p, \cdot\rangle \in\left(\mathrm{T}_{q} \mathbb{R}^{3}\right)^{*}$. Temos que

$$
\begin{aligned}
\mu^{\eta}\left(\alpha_{q}\right) & =\alpha_{q}\left(X^{\eta}(q)\right) \\
& =\alpha_{q}\left(\left.\frac{\mathrm{d}}{\mathrm{d} t}\left(\psi^{l i n}\right)^{q}(\exp (t \eta))\right|_{t=0}\right) \\
& =\alpha_{q}\left(\left.\frac{\mathrm{d}}{\mathrm{d} t}(t \eta+q)\right|_{t=0}\right) \\
& =\langle p, \eta\rangle
\end{aligned}
$$

o que coincide com a aplicação momento encontrada no exemplo 2.3.10.

Para o exemplo 2.3.12, considere a ação $\psi^{\text {ang }}: \mathrm{SO}(3) \times \mathbb{R}^{3} \rightarrow \mathbb{R}^{3}$ dada por $\psi^{\text {ang }}(g, q)=g q$. Seu levantamento cotangente é dado por $\Psi_{g}^{a n g}(q, p)=(g q, g p)$. Então, novamente, dados $q \in \mathbb{R}^{3}$, $\eta=f(v) \in \operatorname{Lie}\left(\mathbb{R}^{3}\right)=\mathbb{R}^{3}$, onde $f$ é a função definida no lema 2.3.11, e $\alpha_{q}=\langle p, \cdot\rangle \in\left(\mathrm{T}_{q} \mathbb{R}^{3}\right)^{*}$, temos que

$$
\begin{aligned}
\mu^{f(v)}\left(\alpha_{q}\right) & =\alpha_{q}\left(X^{\eta}(q)\right) \\
& =\alpha_{q}\left(\left.\frac{\mathrm{d}}{\mathrm{d} t}\left(\psi^{a n g}\right)^{q}(\exp (t f(v)))\right|_{t=0}\right) \\
& =\alpha_{q}\left(\left.\frac{\mathrm{d}}{\mathrm{d} t}\left(e^{t f(v)} q\right)\right|_{t=0}\right) \\
& =\alpha_{q}(f(v) q) \\
& =\langle p, f(v) q\rangle \\
& =\langle p, v \times q\rangle \\
& =\langle q \times p, v\rangle
\end{aligned}
$$

que é a mesma aplicação encontrada no exemplo 2.3.10.

O último lema dessa seção trata de ações de subgrupos e ações Hamiltoneanas. Esse lema será usado em diversas ocasiões no capítulo 3 .

Lema 2.3.15. Sejam $G$ grupo de Lie, $(M, \omega)$ variedade simplética e $\psi: G \times M \rightarrow M$ ação Hamiltoneana com aplicação momento $\mu: M \rightarrow \mathfrak{g}^{*}$. Se $H$ é subgrupo de Lie de $G$, com $\mathfrak{h}=\operatorname{Lie}(H)$ e $i: \mathfrak{h} \rightarrow \mathfrak{g}$ é a inclusão, então a ação $\left.\psi\right|_{H \times M}$ é Hamiltoneana com aplicação momento $i_{\mathfrak{h}}^{*} \circ \mu$.

Demonstração. Queremos provar que $X_{\left(i_{\mathfrak{h}}^{*} \circ \mu\right)^{\eta}}=X^{\eta}$ para todo $\eta \in \mathfrak{h}$. Como $\omega$ é não degenerada, basta provar $i_{X_{(i \mathfrak{h} \circ \mu) \eta}^{*}} \omega=i_{X \eta} \omega$.

Seja então $v_{p} \in \mathrm{T} M$ e $\alpha:(-\epsilon, \epsilon) \rightarrow M$ curva tal que $\alpha(0)=p$ e $\alpha^{\prime}(0)=v_{p}$. Temos que

$$
\begin{aligned}
\omega_{p}\left(X_{\left(i_{\mathfrak{h}}^{*} \circ \mu\right)^{\eta}}(p), v_{p}\right) & =\mathrm{d}\left(\left(i_{\mathfrak{h}}^{*} \circ \mu\right)^{\eta}\right)_{p}\left(v_{p}\right) \\
& =\left.\frac{\mathrm{d}}{\mathrm{d} t}\left(i_{\mathfrak{h}}^{*} \circ \mu\right)^{\eta}(\alpha(t))\right|_{t=0} \\
& =\frac{\mathrm{d}}{\mathrm{d} t}\left\langle\left. i_{\mathfrak{h}}^{*}(\mu(\alpha(t)), \eta\rangle\right|_{t=0}\right. \\
& =\frac{\mathrm{d}}{\mathrm{d} t}\left\langle\left.\left(\mu(\alpha(t)), i_{\mathfrak{h}}(\eta)\right\rangle\right|_{t=0}\right. \\
& =\mathrm{d} \mu^{\eta}\left(v_{p}\right) \\
& =\omega\left(X^{\eta}(p), v_{p}\right)
\end{aligned}
$$


Seguiremos com um exemplo para ilustrar o lema 2.3.15. Esse exemplo ilustra também uma parte da aplicação do teorema de Kirillov-Kostant-Sourieau 2.4.1 que será apresentado na seção 2.4, na prova do teorema de Shur e Horn no capítulo 3.

Exemplo 2.3.16 (Ação de um subgrupo na órbita coadjunta). Seja $G$ um grupo de Lie com álgebra de Lie $\mathfrak{g}$. Considere sua ação coadjunta $\mathrm{Ad}^{*}: G \times \mathfrak{g}^{*} \rightarrow \mathfrak{g}^{*}$. Usaremos aqui o teorema de Kirillov-Kastant-Sourieau que será provado na próxima seção. Em suma, o teorema afirma que, para todo $\zeta \in \mathfrak{g}^{*}$, a órbita $M=\operatorname{Ad}^{*}(G) \zeta$ é uma variedade simplética e que $\psi=\left.\operatorname{Ad}^{*}\right|_{G \times M}$ é ação Hamiltoneana com aplicação momento $i_{M}: M \mathfrak{g}^{*}$ a inclusão.

Considere então $H$ subgrupo de Lie de $G$ com álgebra de Lie $\mathfrak{h}$, com $i_{\mathfrak{h}}: \mathfrak{h} \rightarrow \mathfrak{g}$ a inclusão. O lema 2.3.15 nos garante que a ação $\tilde{\psi}=\left.\psi\right|_{H \times M}$ é Hamiltoneana com aplicação momento $\tilde{\mu}=i_{M}^{*} \circ \mu$. Considere uma métrica $\langle\cdot, \cdot\rangle$ em $\mathfrak{g}$ e defina $\theta: \mathfrak{g} \rightarrow \mathfrak{g}^{*}$ tal que $\theta(\eta)=\langle\eta, \cdot\rangle$. Vamos provar que $\tilde{\mu}=i_{\mathfrak{h}}^{*} \circ \pi_{\theta(\mathfrak{h})}$, onde $\pi_{\theta(\mathfrak{h})}: \theta(\mathfrak{h}) \rightarrow \mathfrak{g}$ é a projeção ortogonal.

Basta provar que $i_{\mathfrak{h}}^{*} \circ i_{M}=i_{\mathfrak{h}}^{*} \circ \pi_{\theta(\mathfrak{h})}$. Note, no entanto, que, se $\xi \in M$

$$
i_{\mathfrak{h}}^{*} \circ i_{M}(\xi)=\left.i_{\mathfrak{h}}^{*} \circ \pi_{\theta(\mathfrak{h})}(\xi) \Leftrightarrow i_{M}(\xi)\right|_{\mathfrak{h}}=\left.\xi\right|_{\mathfrak{h}}=\left.\pi_{\theta(\mathfrak{h})}(\xi)\right|_{\mathfrak{h}}
$$

Logo, é suficiente mostrar que $\xi(\eta)=\pi_{\theta(\mathfrak{h})}(\xi)(\eta)$, para todo $\xi \in \mathfrak{g}^{*}$ e $\eta \in \mathfrak{h}$. Se provarmos que vale $\pi_{\theta(\mathfrak{h})} \circ \theta=\theta \circ \pi_{\mathfrak{h}}$, onde $\pi_{\mathfrak{h}}: \mathfrak{g} \rightarrow \mathfrak{h}$ é projeção ortogonal sobre $\mathfrak{h}$, o lema estará provado pois

$$
\begin{aligned}
\xi(\eta) & =\left\langle\theta^{-1}(\xi), \eta\right\rangle \\
& =\left\langle\pi_{\mathfrak{h}}\left(\theta^{-1}(\xi)\right), \eta\right\rangle+\left\langle\theta^{-1}(\xi)-\pi_{\mathfrak{h}}\left(\theta^{-1}(\xi)\right), \eta\right\rangle \\
& =\left\langle\pi_{\mathfrak{h}}\left(\theta^{-1}(\xi)\right), \eta\right\rangle \\
& =\left(\theta \circ \pi_{\mathfrak{h}}\right)\left(\theta^{-1}(\xi)\right)(\eta) \\
& =\pi_{\theta(\mathfrak{h})}(\xi)(\eta)
\end{aligned}
$$

Para ver que $\pi_{\theta(\mathfrak{h})} \circ \theta=\theta \circ \pi_{\mathfrak{h}}$, note que, se $\xi \in \mathfrak{h}$, então

$$
\theta \circ \pi_{\mathfrak{h}}(\xi)=\theta(\xi)=\pi_{\theta(\mathfrak{h})} \circ \theta(\xi)
$$

Por outro lado, se $\xi \in \mathfrak{h}^{\perp}$ então $\theta(\xi) \in \theta(\mathfrak{h})^{\perp}$, logo

$$
\left(\pi_{\theta(\mathfrak{h})} \circ \theta\right)(\xi)=0=\left(\theta \circ \pi_{\mathfrak{h}}\right)(\eta)
$$

O resultado então segue da linearidade de $\theta$ e $\pi_{\theta(\mathfrak{h})}$.

\subsection{Teorema de Kirillov-Kostant-Sourieau}

Um resultado interessante, que nos dá mais um exemplo de variedade simplética e ação Hamiltoneana, é o teorema de Kirillov-Kostant-Sourieau sobre órbitas coadjuntas. O teorema diz que toda órbita coadjunta admite estrutura simplética para a qual a ação coadjunta é Hamiltoneana. Mais especificamente, o enunciado do teorema é

Teorema 2.4.1. Seja $G$ um grupo de Lie e $\mathrm{Ad}^{*}: G \times \mathfrak{g}^{*} \rightarrow \mathfrak{g}^{*}$ sua ação coadjunta. Se $M=\operatorname{Ad}^{*}(G) \zeta$ é uma órbita da ação coadjunta, então existe $\omega \in \Omega^{2}(M)$ forma simplética, denominada forma de Kirillov-Kostant-Sourieau, definida por

$$
\omega_{\xi}\left(X^{\eta_{1}}(\xi), X^{\eta_{2}}(\xi)\right)=\xi\left(\left[\eta_{1}, \eta_{2}\right]\right)
$$

onde $\eta_{1}, \eta_{2} \in \mathfrak{g}$ e $\xi \in \mathfrak{g}^{*}$. Além disso, a restrição da ação coadjunta $\left.\mathrm{Ad}^{*}\right|_{G \times M}$ é Hamiltoneana com aplicação momento dada pela inclusão $i_{M}: M \rightarrow \mathfrak{g}^{*}$.

A prova desse teorema será feita em partes. Primeiro provaremos que a forma de KirillovKostant-Sourieau é realmente uma forma simplética 
Lema 2.4.2. Sejam $M=\operatorname{Ad}^{*}(G) \zeta$ e $\omega \in \Omega^{2}(M)$ como definidos no teorema 2.4.1. Se $\xi \in M$, então vale que

1. $\omega_{\xi}: \mathrm{T}_{\xi} M \oplus \mathrm{T}_{\xi} M \rightarrow \mathbb{R}$ está bem definida.

2. $\omega_{\xi}$ é bilinear.

3. $\omega_{\xi}$ é anti-simétrica.

4. $\omega_{\xi}$ é não-degenerada.

5. $\omega_{\xi}$ é fechada.

Demonstração. Para provar o item 1 , note primeiro que $M$ é uma órbita de uma ação de $G$, $\operatorname{logo}$ todo elemento de $\mathrm{T}_{\xi} M$ pode ser escrito como $X^{\eta}(\xi)$ para algum $\eta \in \mathfrak{g}$. Dessa forma vemos que $\omega_{\xi}$ realmente está definida no domínio indicado. Para verificar que não há ambiguidade em sua definição, vamos primeiro mostrar uma igualdade que nos ajudará no restante da prova do lema.

$$
\begin{aligned}
X^{\eta}(\xi) & =\left.\frac{\mathrm{d}}{\mathrm{d} t}\left(\operatorname{Ad}^{*}(\exp (t \eta)) \xi\right)\right|_{t=0} \\
& =\left.\frac{\mathrm{d}}{\mathrm{d} t} \xi \circ \operatorname{Ad}(\exp (-t \eta))\right|_{t=0} \\
& =\left.\xi \circ \frac{\mathrm{d}}{\mathrm{d} t} \operatorname{Ad}(\exp (-t \eta))\right|_{t=0} \\
& =\xi \circ \operatorname{ad}(-\eta) \\
& =-\xi \circ \operatorname{ad}(\eta) \\
& =\xi([\eta, \cdot])
\end{aligned}
$$
dá

Sejam $\eta^{1}, \hat{\eta}^{1}, \eta^{2} \in \mathfrak{g}$, tais que $X^{\eta^{1}}(\xi)=X^{\hat{\eta}^{1}}(\xi)$ então temos que $\xi\left(\left[\eta^{1}, \cdot\right]\right)=\xi\left(\left[\hat{\eta}^{1}, \cdot\right]\right)$, o que nos

$$
\begin{aligned}
\omega_{\xi}\left(X^{\eta^{1}}(\xi), X^{\eta^{2}}(\xi)\right) & =\xi\left(\left[\eta^{1}, \eta^{2}\right]\right) \\
& =\xi\left(\left[\hat{\eta}^{1}, \eta^{2}\right]\right) \\
& =\omega_{\xi}\left(X^{\hat{\eta}^{1}}(\xi), X^{\eta^{2}}(\xi)\right)
\end{aligned}
$$

Usando que $X^{\eta}(\xi)=-\xi([\cdot, \eta])$ provamos o mesmo para a segunda coordenada e está provado o item 1.

O item 2 vem do fato de o colchete ser bilinear e do item 1 do lema 2.2.2, que garante que $\mathfrak{g} \ni \eta \mapsto X^{\eta}(\xi) \mathfrak{X}(M)$ é linear.

O item 3 vem direto do fato de que o colchete é anti-simétrico.

Para provar o item 4 , observe que se $\omega_{\xi}\left(X^{\eta^{1}}(\xi), X^{\eta^{2}}(\xi)\right)=0$, para todo $\eta^{2} \in \mathfrak{g}$, temos então

$$
\begin{aligned}
0 & =\omega_{\xi}\left(X^{\eta^{1}}(\xi), X^{\eta^{2}}(\xi)\right) \\
& =\xi\left(\left[\eta^{1}, \eta^{2}\right]\right)
\end{aligned}
$$

para todo $\eta^{2} \in \mathfrak{g}$. Logo $X^{\eta^{1}}(\xi)=\xi\left(\left[\eta^{1}, \cdot\right]\right)=0$.

O item 5 é o mais delicado do lema. Vamos começar com a seguinte observação. Sejam $\eta^{1}, \eta^{2} \in \mathfrak{g}$ e $\xi \in \mathfrak{g}^{*}$, podemos extender esses elementos como campos invariantes à esquerda fazendo $\tilde{\eta}_{g}^{1}=\mathrm{d} L_{g} \eta^{1}$, $\tilde{\eta}_{g}^{2}=\mathrm{d} L_{g} \eta^{2}, \tilde{\xi}_{g}=\mathrm{d} L_{g^{-1}}^{*} \xi$. Note que a função $\tilde{\xi} \circ \tilde{\eta}^{j}$ é constante, $j=1,2$. Em particular, $X \cdot \xi \circ \eta^{j}=0$, para todo $X \in \mathfrak{g}$. 
Então

$$
\begin{aligned}
\mathrm{d} \tilde{\xi}_{e}\left(\tilde{\eta}_{e}^{1}, \tilde{\eta}_{e}^{2}\right) & =\tilde{\eta}_{e}^{2} \cdot \tilde{\xi}\left(\tilde{\eta}^{1}\right)-\tilde{\eta}_{e}^{2} \cdot \tilde{\xi}\left(\tilde{\eta}^{1}\right)-\tilde{\xi}_{e}\left(\left[\eta^{1}, \eta^{2}\right]\right) \\
& =-\xi\left(\left[\eta^{1}, \eta^{2}\right]\right) \\
& =-\omega_{\xi}\left(X^{\eta^{1}}(\xi), X^{\eta^{2}}(\xi)\right)
\end{aligned}
$$

O leitor pode se sentir tentado nesse momento a aplicar o operador diferencial a ambos os lados e concluir que a forma é exata. Entretanto, as formas d $\tilde{\xi}$ e $\omega$ tem domínios diferentes $\left(\mathrm{d} \tilde{\xi} \in \Omega^{2}(G)\right.$, $\omega \in \Omega(M))$ e, portanto, o operador d é diferente para cada uma delas. Para circundar esse problema, escreva $\psi=\operatorname{Ad}^{*}$ e, para cada $\xi \in \mathfrak{g}^{*}$ faça $\psi^{\xi}: G \rightarrow M$ função definida por $\psi^{\xi}(g)=\operatorname{Ad}^{*}(g) \xi$ como definido no início do capítulo. Se provarmos que o pull-back de $\omega$ por $\psi^{\xi}$ coincide com $-\mathrm{d} \tilde{\xi}$, teremos que

$$
\begin{aligned}
\left(\psi^{\xi}\right)^{*} \mathrm{~d} \omega & =\mathrm{d}\left(\left(\psi^{\xi}\right)^{*} \omega\right) \\
& =-\mathrm{d}(\mathrm{d} \tilde{\xi}) \\
& =0
\end{aligned}
$$

Como $\psi^{\xi}$ é uma submersão, pois $\mathrm{d}\left(\psi^{\xi}\right)_{e}(\eta)=X^{\eta}(\xi)$, teremos então que $\mathrm{d} \omega=0$.

Vamos então provar que $\psi^{*} \omega=\mathrm{d} \xi$. Primeiro mostraremos que as duas formas coincidem na identidade

$$
\begin{aligned}
\left(\left(\psi^{\xi}\right)^{*} \omega\right)_{e}\left(\eta^{1}, \eta^{2}\right) & =\omega_{\xi}\left(\mathrm{d}\left(\psi^{\xi}\right)_{e} \eta^{1}, \mathrm{~d}\left(\psi^{\xi}\right)_{e} \eta^{2}\right) \\
& =\omega_{\xi}\left(X^{\eta^{1}}(\xi), X^{\eta^{2}}(\xi)\right) \\
& =\mathrm{d} \tilde{\xi}_{e}\left(\eta^{1}, \eta^{2}\right)
\end{aligned}
$$

No entanto, o item 2 do lema 2.2.2 nos diz que, para todo campo invariante à esquerda $\tilde{\eta}$ em $G$, temos

$$
\begin{aligned}
\mathrm{d}\left(\psi^{\xi}\right)_{g} \tilde{\eta}_{g} & =\left.\frac{\mathrm{d}}{\mathrm{d} t} \psi\left(g \exp \left(t \tilde{\eta}_{e}\right), \xi\right)\right|_{t=0} \\
& =\left.\frac{\mathrm{d}}{\mathrm{d} t} \psi\left(g, \psi\left(\exp \left(t \tilde{\eta}_{e}\right), \xi\right)\right)\right|_{t=0} \\
& =\left.\mathrm{d} \psi_{g} \circ \frac{\mathrm{d}}{\mathrm{d} t} \psi\left(\exp \left(t \tilde{\eta}_{e}\right), \xi\right)\right|_{t=0} \\
& =\mathrm{d} \psi_{g} \circ X^{\tilde{\eta}_{e}}(\xi) \\
& =X^{\operatorname{Ad}(g) \tilde{\eta}_{e}} \circ \psi_{g} \\
& =X^{\operatorname{Ad}(g) \tilde{\eta}_{e}}\left(\operatorname{Ad}^{*}(g) \xi\right)
\end{aligned}
$$

Isso implica que

$$
\begin{aligned}
\left(\left(\psi^{\xi}\right)^{*} \omega\right)_{g}\left(\tilde{\eta}_{g}^{1}, \tilde{\eta}_{g}^{2}\right) & =\omega_{\psi^{\xi}(g)}\left(\mathrm{d} \psi_{g} \tilde{\eta}_{g}^{1}, \mathrm{~d} \psi_{g} \tilde{\eta}_{g}^{2}\right) \\
& =\omega_{\operatorname{Ad}^{*}(g) \xi}\left(X^{\operatorname{Ad}(g) \eta^{1}}\left(\operatorname{Ad}^{*}(g) \xi\right), X^{\operatorname{Ad}(g) \eta^{2}}\left(\operatorname{Ad}^{*}(g) \xi\right)\right) \\
& =\operatorname{Ad}^{*}(g) \xi\left(\left[\operatorname{Ad}(g) \eta^{1}, \operatorname{Ad}(g) \eta^{2}\right]\right) \\
& =\xi\left(\operatorname{Ad}\left(g^{-1}\right) \operatorname{Ad}(g)\left[\eta^{1}, \eta^{2}\right]\right) \\
& =\xi\left(\left[\eta^{1}, \eta^{2}\right]\right) \\
& =\mathrm{d} \tilde{\xi}_{g}\left(\tilde{\eta}_{g}^{1}, \tilde{\eta}_{g}^{2}\right)
\end{aligned}
$$

concluindo a prova. 
O lema a seguir prova que $\mu=i_{M}: M \rightarrow \mathfrak{g}^{*}$ é aplicação momento para a ação coadjunta

Lema 2.4.3. Sejam $M=\operatorname{Ad}^{*}(G) \zeta$ e $\omega$ a forma de Kostant-Kirillov-Sourieau. Se escrevermos $\mu=i_{M}: M \rightarrow \mathfrak{g}^{*}$ é a inclusão, então vale que

1. $X_{\mu} \xi=X^{\xi}, \forall \xi \in \mathfrak{g}$

2. $i_{M}$ é equivariante, i.e., $\left.i_{M} \circ \operatorname{Ad}^{*}(g)\right|_{M}=\operatorname{Ad}^{*}(g) \circ i_{M}$

Demonstração. Para provar o item 1, basta mostrar que $i_{X_{\left(i_{M}\right)} \eta} \omega=i_{X} \eta \omega$. Sejam $\eta, \tilde{\eta} \in \mathfrak{g}$ e $\xi^{*} \in \mathfrak{g}^{*}$. Note que $\left(i_{M}\right)^{\eta}(\xi)=i_{M}(\xi)(\eta)=\xi(\eta)$. Temos então que

$$
\begin{aligned}
\omega_{\xi}\left(X_{\left(i_{M}\right)^{\eta}}(\xi), X^{\tilde{\eta}}(\xi)\right) & =\left(\mathrm{d}\left(i_{M}\right)^{\eta}\right)_{\xi}\left(X^{\tilde{\eta}}(\xi)\right) \\
& =\left.\frac{\mathrm{d}}{\mathrm{d} t}\left(i_{M}\right)^{\eta}\left(\operatorname{Ad}^{*}(\exp t \tilde{\eta}) \xi\right)\right|_{t=0} \\
& =\left.\frac{\mathrm{d}}{\mathrm{d} t}\left(\operatorname{Ad}^{*}(\exp t \tilde{\eta}) \xi\right)(\eta)\right|_{t=0} \\
& =\left.\frac{\mathrm{d}}{\mathrm{d} t} \xi \circ \operatorname{Ad}(\exp (-t \tilde{\eta}))(\eta)\right|_{t=0} \\
& =\xi \circ \operatorname{ad}(-\tilde{\eta} \eta) \\
& =\xi([\eta, \tilde{\eta}]) \\
& =\omega_{\xi}\left(X^{\eta}(\xi), X^{\tilde{\eta}}(\xi)\right)
\end{aligned}
$$

O item 2 é obviamente verdadeiro.

\subsection{Redução Simplética}

Para ilustrar a utilidade da aplicação momento, apresentaremos nesta seção o teorema de Marsden-Weinstein-Meyer sobre redução simplética, que utiliza a aplicação momento para reduzir o número de dimensões do problema. Essa seção é baseada em [1].

\subsubsection{Resultados preliminares}

Aqui apresentaremos os ingredientes básicos usados na prova do teorema de redução simplética. Começaremos definindo o conceito de anuladorda álgebra linear.

Definição 2.5.1. Seja $V$ um espaço vetorial e $W$ subconjunto de $V$. $O$ anulador de $W$ é o subespaço $W^{0}=\left\{f \in V^{*} \mid f(w)=0, \forall w \in W\right\}$

O primeiro lema estabelece uma relação entre o kernel da aplicação momento, que corresponde ao espaço tangente dos seus conjuntos de nível, e o espaço tangente das órbitas da ação.

Lema 2.5.2. Seja $\psi: G \times M \rightarrow M$ ação hamiltoneana. Se $G_{p}$ é o grupo de isotropia de $p$ e $\mathfrak{g}_{p}=\operatorname{Lie}\left(G_{p}\right)$ e $\mathcal{O}_{p}$ é a órbita de $G$ que passa por $p$, então:

$$
\begin{aligned}
\operatorname{Ker} \mathrm{d} \mu_{p} & =\left(\mathrm{T}_{p} \mathcal{O}_{p}\right)^{\omega_{p}} \\
\operatorname{Im} \mathrm{d} \mu_{p} & =\mathfrak{g}_{p}^{0}
\end{aligned}
$$

onde $\mathfrak{g}_{p}^{0}$ é o anulador de $\mathfrak{g}_{p}$. 
Demonstração. Observe primeiro que

$$
\begin{aligned}
\operatorname{dim} \mathfrak{g}_{p}^{0} & =\operatorname{dim} \mathfrak{g}-\operatorname{dim} \mathfrak{g}_{p} \\
\operatorname{dim}\left(\mathrm{T}_{p} \mathcal{O}_{p}\right)^{\omega_{p}} & =\operatorname{dim} \mathrm{T}_{p} M-\left(\operatorname{dim} \mathfrak{g}-\operatorname{dim} \mathfrak{g}_{p}\right)
\end{aligned}
$$

onde a segunda igualdade vem da proposição 1.2.13. Assim

$$
\operatorname{dim} \mathfrak{g}_{p}^{0}+\operatorname{dim}\left(\mathrm{T}_{p} \mathcal{O}_{p}^{\omega_{p}}\right)=\operatorname{dim} \mathrm{T}_{p} M=\operatorname{dim}\left(\operatorname{Ker} \mathrm{d} \mu_{p}\right)+\operatorname{dim}\left(\operatorname{Imd} \mu_{p}\right)
$$

Se provarmos que $\operatorname{Im} \mathrm{d} \mu_{p} \subset \mathfrak{g}_{p}^{0}$ e $\operatorname{Ker} \mathrm{d} \mu_{p} \subset \mathrm{T}_{p} \mathcal{O}_{p}^{\omega_{p}}$ então o resultado sai por um argumento de dimensão.

Observe que, $\forall \eta \in \mathfrak{g}$ e $\forall v \in T_{p} M$

$$
\omega_{p}\left(\left(X^{\eta}\right)_{p}, v\right)=\left\langle d \mu_{p}(v), \eta\right\rangle
$$

Logo se $v \in \operatorname{Ker} \mathrm{d} \mu_{p}$, então $\omega_{p}\left(\left(X^{\eta}\right)_{p}, v\right)=0, \forall \eta \in \mathfrak{g}$. Portanto temos Ker $\mathrm{d} \mu_{p} \subset\left(\mathrm{T}_{p} \mathcal{O}_{p}\right)^{\omega_{p}}$.

Por outro lado, para todo $\eta \in \mathfrak{g}_{p}$ temos

$$
\begin{aligned}
X^{\eta}(p) & =\left.\frac{\mathrm{d}}{\mathrm{d} t} \psi(\exp (t \eta), p)\right|_{t=0} \\
& =\left.\frac{\mathrm{d}}{\mathrm{d} t} p\right|_{t=0} \\
& =0
\end{aligned}
$$

Logo $\left\langle d \mu_{p}(v), \eta\right\rangle=0$ para todo $v \in \mathrm{T}_{p} M$, o que implica $\operatorname{Im} \mathrm{d} \mu_{p} \subset \mathfrak{g}_{p}^{0}$.

Lema 2.5.3. Se $(V, \omega)$ é um espaço vetorial simplético e $W$ é um subespaço isotrópico de $V$, então $\omega$ induz uma forma simplética $\omega_{\text {red }}$ em $W^{\omega} / W$.

Demonstração. Se $u, v \in W^{\omega}$, defina $\omega_{\text {red }}([u],[v])=\omega(u, v)$. Para ver que $\omega_{\text {red }}$ está bem definida, basta observar que se $\tilde{u} \in[u]$ e $\tilde{v} \in[v]$ então $\tilde{u}=u+w, \tilde{v}=v+z, \operatorname{com} w, z \in W$ e, como $W \subset W^{\omega}$

$$
\omega(u+w, v+z)=\omega(u, v)+\omega(u, z)+\omega(w, v)+\omega(w, z)=\omega(u, v)
$$

Para ver que $\omega_{\text {red }}$ é não-degenerada, tome $u \in W^{\omega}$ tal que $\omega_{\text {red }}([u],[v])=\omega(u, v)=0, \forall v \in W^{\omega}$. Então $u \in\left(W^{\omega}\right)^{\omega}=W, \operatorname{logo}[u]=0$.

O lema seguinte será apenas enunciado e não provado, por se tratar de um resultado de teoria geral de variedades, e não de variedades simpléticas.

Lema 2.5.4. Se $G$ é compacto e age livremente sobre $M$, então $M / G$ é uma variedade e o mapa $\pi: M \rightarrow M / G$ é um G-fibrado principal.

\subsubsection{Teorema de Marsden-Weinstein-Meyer}

Teorema 2.5.5. Sejam $(M, \omega)$ variedade simplética, $G$ um grupo de Lie compacto com ação Hamiltoneana sobre $M$ com aplicação momento $\mu$ e $k \in \mu(M)$. Suponha que $G$ age livremente sobre $\mu^{-1}(k)$, então, se $i: \mu^{-1}(k) \rightarrow M$ é a inclusão, temos que:

- $M_{\text {red }}=\mu^{-1}(k) / G$ é variedade

- A aplicação $\pi: \mu^{-1}(k) \rightarrow M_{\text {red }}$ é um G-fibrado principal

- Existe $\omega_{\text {red }}$ forma simplética em $M_{\text {red }}$ tal que $i^{*} \omega=\pi^{*} \omega_{\text {red }}$ 
Demonstração. Como $G$ age livremente sobre $\mu^{-1}(k)$ temos que $G_{p}=\{0\}$ para todo $p \in \mu^{-1}(k)$ o que implica $\mathfrak{g}_{p}=\operatorname{Lie}\left(G_{p}\right)=0$. O lema 2.5.2 nos garante então que d $\mu_{p}$ é sobrejetora. Logo $k$ é valor regular de $\mu$ e $\mu^{-1}(k)$ é subvariedade de $M$ com codimensão igual à dimensão de $G$. Com isso, o lema 2.5.4 nos dá os dois primeiros itens.

Para provar o terceiro item, observe que, se $p \in \mu^{-1}(k)$, então, pelo lema 2.5.2 temos que $\left(\mathrm{T}_{p} \mathcal{O}_{p}\right)^{\omega_{p}}=\operatorname{Ker} \mathrm{d} \mu_{p}=\mathrm{T}_{p} \mu^{-1}(k)$. Mas como $G$ age sobre $\mu^{-1}(k)$ temos $\mathrm{T}_{p} \mathcal{O}_{p} \subset \mathrm{T}_{p} \mu^{-1}(k)=$ $\left(\mathrm{T}_{p} \mathcal{O}_{p}\right)^{\omega_{p}}$, i.e., $\mathrm{T}_{p} \mathcal{O}_{p}$ é isotrópico.

O lema 2.5.3 nos garante que existe uma estrutura linear simplética em $\mathrm{T}_{p} \mu^{-1}(0) / \mathrm{T}_{p} \|_{p}=$ $\mathrm{T}_{[p]} M_{r e d}$ induzida por $\omega_{p}$. Logo, o mesmo lema nos dá um espaço vetorial simplético $\left(\mathrm{T}_{[p]} M_{r e d},\left(\omega_{r e d}\right)_{p}\right)$. Vamos agora mostrar que $\left(\omega_{\text {red }}\right)_{p}$ depende apenas de $[p]$, ou seja, poderemos denotar $\left(\omega_{\text {red }}\right)_{p}=$ $\left(\omega_{\text {red }}\right)_{[p]}$.

De fato, observe que existe $g \in G$ tal que $\psi(g, p)=\tilde{p}$ e que

$$
\begin{aligned}
\mathrm{d} \psi_{g}\left(\mathrm{~T}_{p} \mu^{-1}(k)\right) & =\mathrm{T}_{\tilde{p}} \mu^{-1}(k) \\
\mathrm{d} \psi_{g}\left(\mathrm{~T}_{p} \mathcal{O}_{p}\right) & =\mathrm{T}_{\tilde{p}} \mathcal{O}_{\tilde{p}}
\end{aligned}
$$

logo podemos fazer a identificação $[u]=\left[\mathrm{d} \psi_{g}(u)\right]$ para $u \in \mathrm{T}_{p} \mu^{-1}(k)$.

Assim se $\omega_{r e d}$ é a forma induzida escolhendo o ponto $p$ e $\tilde{\omega}_{r e d}$ a forma obtida pelo ponto $\tilde{p}$, para todos $u, v \in T_{p} \mu^{-1}(k)$, temos que

$$
\begin{aligned}
\left(\omega_{\text {red }}\right)_{p}([v],[u]) & =\omega(u, v) \\
& =\omega\left(\mathrm{d} \psi_{g}(u), \mathrm{d} \psi_{g}(v)\right) \\
& =\left(\tilde{\omega}_{r e d}\right)_{\tilde{p}}\left(\left[\mathrm{~d} \psi_{g}(u)\right],\left[\mathrm{d} \psi_{g}(v)\right]\right)
\end{aligned}
$$

Já mostramos que a cada ponto $p \in M_{\text {red }}$ existe uma estrutura linear simplética $\left(\omega_{\text {red }}\right)_{p}$ em $\mathrm{T}_{p} M_{\text {red }}$. Vamos agora provar que $\omega_{\text {red }}$ é uma forma simplética em $M$. O fato de $\left(\omega_{\text {red }}\right)_{p}$ ser simplética para todo $p \in M_{\text {red }}$ prova a biliearidade, anti-simetria e não degenerescência, resta apenas provar $\omega_{\text {red }}$ é fechada. Por construção, temos $i^{*} \omega=\pi^{*} \omega_{\text {red }}$, logo

$$
\begin{aligned}
\pi^{*} d \omega_{\text {red }} & =d \pi^{*} \omega_{\text {red }} \\
& =d i^{*} \omega \\
& =i^{*} d \omega \\
& =0
\end{aligned}
$$

Como $\pi^{*}$ é injetora, temos que $\omega_{\text {red }}$ é fechada e, portanto, simplética.

A seguir apresentaremos um exemplo para ilustrar o uso da redução simplética de Marsden provando que o espaço projetivo $\mathbb{C P}^{1}$ é uma variedade simplética. Na verdade, uma pequena adaptação pode ser feita para provar que qualquer $\mathbb{C P}^{n}$ é simplético.

Exemplo 2.5.6 (Redução para $\left.\mathbb{C P}^{1}\right)$. Considere a ação $\psi: S^{1} \times \mathbb{C}^{2} \rightarrow \mathbb{C}^{2}$ dada por $\psi\left(e^{2 \pi i t},\left(z_{1}, z_{2}\right)\right)=$ $\left(e^{2 \pi i t} z_{1}, e^{2 \pi i t} z_{2}\right)$. Essa ação pode ser vista como uma restrição da ação introduzida no exemplo 2.3.9, com $n=2$, ao subtoro diagonal $\left\{\left(e^{2 \pi i t}, e^{2 \pi i t}\right) \mid t \in \mathbb{R}\right\} \subset T^{2}$. Assim, aplicando o lema 2.3.15, temos que $\psi$ é Hamiltoneana com aplicação momento $\mu: \mathbb{C}^{2} \rightarrow \mathbb{R}^{*}$ dada por

$$
\mu\left(z_{1}, z_{2}\right)=\left\langle\left(\left|z_{1}\right|^{2}+k_{1},\left|z_{2}\right|^{2}+k_{2}\right), \cdot\right\rangle
$$

onde $k_{1}, k_{2} \in \mathbb{R}$ são constantes que tomaremos como zero. Temos então que $\mu^{-1}\left(\operatorname{Id}_{\mathbb{R}}\right)$ é a esfera de raio $1 \mathrm{em} \mathbb{C}^{2}$. Como $\psi_{g}$ é sempre uma rotação e $\psi$ é uma ação livre, temos que $\psi$ age livremente em $\mu^{-1}\left(\operatorname{Id}_{\mathrm{R}}\right.$. Assim, pelo teorema 2.5 .5 , temos que $\mu^{-1}\left(\operatorname{Id}_{\mathrm{R}} / S^{1}=\mathbb{C P}^{1}\right.$ é uma variedade simplética. 


\subsubsection{Redução para Grupos Produto}

Discutiremos aqui como usar o teorema 2.5.5 para fazer uma redução simplética em grupos produto. A idéia é, dados $G=G_{1} \times G_{2}$ grupo de Lie produto, e uma ação Hamiltoneana $\psi$ : $G \times M \rightarrow M$, fazer a redução apenas segundo $G_{1}$ e analisar a ação de $G_{2}$ no quociente.

Proposição 2.5.7. Sejam $(M, \omega)$ variedade simplética, $G_{1}$ e $G_{2}$ grupos de Lie e $G=G_{1} \times G_{2}$ grupo de Lie com a estrutura produto. Sejam $\psi: G \times M \rightarrow M$ ação Hamiltoneana com aplicação momento $\mu$. Então $\psi^{1}=\left.\psi\right|_{\left(G_{1} \times\left\{e_{G_{2}}\right\}\right) \times M}$ e $\psi^{2}=\left.\psi\right|_{\left(\left\{e_{G_{1}}\right\} \times G_{2}\right) \times M}$ são ações Hamiltoneanas com aplicação momento $\mu_{1}$ e $\mu_{2}$. Além disso, se $G_{1}$ age livremente em $\mu_{1}^{-1}(k), k \in \mu_{1}(M)$, então existe ação Hamiltoneana de $G_{2}$ em $M_{1}=\mu_{1}^{-1}(k) / G_{1}$ com relação à forma simplética reduzida $\omega_{\text {red }}$ com aplicação momento $\tilde{\mu}_{1}$ tal que o diagrama abaixo comuta.

Demonstração. Se $\mathfrak{g}, \mathfrak{g}_{1}$ e $\mathfrak{g}_{2}$ são as álgebras de Lie de $G, G_{1}$ e $G_{2}$, respectivamente, então o lema 2.3.15 garante que $\psi_{1}$ e $\psi_{2}$ são ações Hamiltoneanas com aplicação momento $\mu_{1}=i_{\mathfrak{g}_{1}}^{*} \circ \mu \mathrm{e}$ $\mu_{2}=i_{\mathfrak{g}_{2}}^{*} \circ \mu$, onde $i_{\mathfrak{g}_{i}}: \mathfrak{g}_{i} \rightarrow \mathfrak{g}$ é a inclusão para $i=1,2$. Em particular, dados $p \in M$ e $\eta_{1} \in \mathfrak{g}_{1}$ temos que

$$
\begin{aligned}
\mu_{1}^{\eta_{1}}(p) & =i_{\mathfrak{g}_{1}}^{*} \circ \mu(p)\left(\eta_{1}\right) \\
& =\mu(p)\left(\eta_{1}\right) \\
& =\mu^{\eta_{1}}(p)
\end{aligned}
$$

Analogamente, se $\eta_{2} \in \mathfrak{g}_{2}$, temos $\mu_{2}^{\eta_{2}}(p)=\mu^{\eta_{2}}(p)$.

Note que as ações $\psi^{1}$ e $\psi^{2}$ comutam, no sentido que, para quaisquer $g_{1} \in G_{1}$ e $g_{2} \in G_{2}$, $\psi_{g_{1}}^{1} \circ \psi_{g_{2}}^{2}=\psi_{g_{2}}^{2} \circ \psi_{g_{1}}^{1}$. Isso implica que $G_{2}$ age em $\mu_{1}^{-1}(k)$. Para ver isso, basta provar que, dados $p \in \mu_{1}^{-1}(k)$ e $\eta_{2} \in \mathfrak{g}_{2}$ elemento da álgebra de Lie de $G_{2},\left.\frac{\mathrm{d}}{\mathrm{d} t} \mu_{1}\left(\psi^{2}\left(\exp _{G_{2}}\left(t \eta_{2}\right), p\right)\right)\right|_{t=0}=0$. Logo, dado $\eta_{1} \in \mathfrak{g}_{1}$ elemento da álgebra de Lie de $G_{1}$, temos

$$
\begin{aligned}
\left.\frac{\mathrm{d}}{\mathrm{d} t} \mu_{1}^{\eta_{1}}\left(\psi^{2}\left(\exp _{G_{2}}\left(t \eta_{2}\right), p\right)\right)\right|_{t=0} & =\mathrm{d}\left(\mu_{1}^{\eta_{1}}\right)_{p}\left(X^{\eta_{2}}(p)\right) \\
& =\omega\left(X_{\mu_{1}^{\eta_{1}}}(p), X^{\eta_{2}}(p)\right) \\
& =\omega\left(X_{\mu_{1}^{\eta_{1}}}(p), X_{\left.\mu_{2}^{\eta_{2}}(p)\right)}\right. \\
& =\left\{\mu_{1}^{\eta_{1}}, \mu_{2}^{\eta_{2}}\right\}(p) \\
& =\left\{\mu^{\eta_{1}}, \mu^{\eta_{2}}\right\}(p) \\
& =\mu^{\left[\eta_{1}, \eta_{2}\right]}(p) \\
& =\mu^{\left[\eta_{1}, 0\right]}(p)+\mu^{\left[0, \eta_{2}\right]}(p) \\
& =0
\end{aligned}
$$

A igualdade $\left[\eta_{1}, \eta_{2}\right]=\left[\eta_{1}, 0\right]+\left[0, \eta_{2}\right]$ vem da definição do colchete produto, usando as decomposições $\eta_{1}=\eta_{1}+0$ e $\eta_{2}=0+\eta_{2}$.

Assim, se $\pi: \mu^{-1}(k) \rightarrow M_{1}$ é a projeção canônica do quociente, podemos definir a ação $\tilde{\psi}^{2}$ de $G_{2}$ em $M_{1}$ tal que $\pi \circ \psi_{g}^{2}=\tilde{\psi}_{g}^{2} \circ \pi$ para todo $g \in G_{2}$.

Por outro lado, um raciocínio análogo nos mostra que $\left.\frac{\mathrm{d}}{\mathrm{d} t} \mu_{2}^{\eta_{2}}\left(\psi^{1}\left(\exp _{G_{1}}\left(t \eta_{1}\right), p\right)\right)\right|_{t=0}=0$, ou seja, que $\mu_{2}$ é constante ao longo das órbitas de $G_{1}$. Isso nos permite definir a função $\tilde{\mu}_{2}: M_{1} \rightarrow \mathfrak{g}_{2}^{*}$ tal que $\tilde{\mu}_{2} \circ \pi=\mu_{2}$.

Vamos agora provar que $\tilde{\mu}_{2}$ é aplicação momento para $\tilde{\psi}^{2}$. Dado $\eta \in \mathfrak{g}_{2}$, denote por $\tilde{X}^{\eta}$ a ação 
infinitesimal de $\tilde{\psi}^{2}$. Veja que, dado $p \in \mu^{-1}(k)$

$$
\begin{aligned}
\tilde{X}^{\eta}([p]) & =\left.\frac{\mathrm{d}}{\mathrm{d} t} \tilde{\psi}^{2}(\exp (t \eta), \pi(p))\right|_{t=0} \\
& =\left.\frac{\mathrm{d}}{\mathrm{d} t} \pi \circ \tilde{\psi}^{2}(\exp (t \eta), p)\right|_{t=0} \\
& =\mathrm{d} \pi \circ X^{\eta}(p)
\end{aligned}
$$

Temos, então, que mantendo $p \in \mu^{-1}(k)$ e tomando $v \in \mathrm{T}_{p} \mu^{-1}(k)$

$$
\begin{aligned}
\omega_{\text {red }}\left(X_{\tilde{\mu}_{2}^{\eta}}([p]),[v]\right) & =\mathrm{d}\left(\tilde{\mu}_{2}^{\eta}\right)_{[p]} \circ \mathrm{d} \pi_{p}(v) \\
& =\mathrm{d}\left(\mu_{2}^{\eta}\right)_{p}(v) \\
& =\omega\left(X_{\mu^{\eta}}(p), v\right) \\
& =\omega\left(X^{\eta}(p), v\right) \\
& =\omega_{r e d}\left(\mathrm{~d} \pi_{p}\left(X^{\eta}(p)\right), \mathrm{d} \pi_{p}(v)\right) \\
& =\omega_{\text {red }}\left(\tilde{X}^{\eta}([p]),[v]\right)
\end{aligned}
$$

o que nos dá $X_{\tilde{\mu}_{2}^{\eta}}([p])=\tilde{X}^{\eta}([p])$. Para provar a equivariância, pela proposição 2.3 .7 basta provar que a aplicação comomento é um homomorfismo de Lie com o colchete de Poisson $\{\cdot, \cdot\}_{\text {red }}=$ $\omega_{\text {red }}\left(X_{(\cdot)}, X_{(\cdot)}\right)$. Considerando $\eta^{1}, \eta^{2} \in \mathfrak{g}_{2}$, temos

$$
\begin{aligned}
\left\{\tilde{\mu}_{2}^{\eta^{1}}, \tilde{\mu}_{2}^{\eta^{2}}\right\}_{\text {red }}([p]) & =\omega_{\text {red }}\left(X_{\tilde{\mu}_{2}^{\eta 1}}([p]), X_{\tilde{\mu}_{2}^{\eta^{2}}}([p])\right) \\
& =\omega\left(X_{\mu_{2}^{\eta^{1}}}(p), X_{\mu_{2}^{\eta^{2}}}(p)\right) \\
& =\left\{\mu_{2}^{\eta^{1}}, \mu_{2}^{\eta^{2}}\right\}(p) \\
& =\mu_{2}^{\left[\eta^{1}, \eta^{2}\right]}(p) \\
& =\tilde{\mu}_{2}^{\left[\eta^{1}, \eta^{2}\right]}([p])
\end{aligned}
$$

O exemplo a seguir exemplifica o uso da redução para grupos produto. Na verdade o uso a seguir é uma versão em dimensão baixa do uso que esse resultado terá na seção 3.4.

Exemplo 2.5.8 (Redução produto para $T^{2}$ agindo em $\mathbb{C}^{2}$ ). Considere a ação $\psi: T^{2} \times \mathbb{C}^{2} \rightarrow \mathbb{C}^{2}$ apresentada no exemplo 2.3.9. Essa ação tem aplicação momento $\mu: \mathbb{C}^{2} \rightarrow\left(\mathbb{R}^{2}\right)^{*}$ dada por

$$
\mu\left(z_{1}, z_{2}\right)=\left\langle\left(\left|z_{1}\right|^{2}+k_{1},\left|z_{2}\right|^{2}+k_{2}\right), \cdot\right\rangle
$$

Podemos decompor $T^{2}$ nos subtoros $T_{1}=\left\{\left(e^{2 \pi i t}, e^{2 \pi i t}\right) \mid t \in \mathbb{R}\right\}$ e $T_{2}=\left\{\left(e^{2 \pi i t}, e^{-2 \pi i t}\right) \mid t \in \mathbb{R}\right\}$. Vimos no exemplo 2.5.6 que a redução de Marsden por $\psi^{1}=\left.\psi\right|_{T_{1} \times \mathbb{C}^{2}}$ nos dá o espaço projetivo $\mathbb{C P}^{1}$. Assim, a proposição 3.4 nos garante que existe uma ação $\tilde{\psi}: T_{2} \times \mathbb{C P}^{1} \rightarrow \mathbb{C P}^{1}$ Hamiltoneana com aplicação momento $\tilde{\mu}: \mathbb{C P}^{1} \rightarrow \mathbb{R}^{*}$ definida de forma que $\tilde{\mu} \circ \pi=i_{\mathfrak{t}_{2}}^{*} \circ \mu$, onde $\mathfrak{t}_{2}$ é a álgebra de Lie de $T_{2}$ e $i_{\mathfrak{t}_{2}}: \mathfrak{t}_{2} \rightarrow \mathbb{R}^{2}$ é a inclusão de $\mathfrak{t}_{2}$ em $\mathbb{R}^{2}$ álgebra de Lie de $T^{2}$ e $\pi: \mu_{1}^{-1}\left(\operatorname{Id}_{\mathrm{R}}\right) \rightarrow \mathbb{C P}^{1}$ é aprojeção sobre o quociente.

\subsection{Condições para Existência e para a Unicidade da Aplicação Comomento}

Já vimos pelos resultados apresentados que a aplicação momento se mostra muito útil em problemas de geometria simplética, por exemplo auxiliando encontrar simetrias de um problema e 
reduzir sua dimensão ou apresentando uma lei de conservação no sistema considerado. Entretanto, tal aplicação nem sempre existe, devido a possíveis obstruções toplógicas para isso.

Nesta seção mostraremos condições necessárias para a existência e para a unicidade da aplicação comomento e, portanto, também da aplicação momento de uma ação simplética. Em resumo mostraremos que toda ação simplética de um grupo com álgebra de Lie semisimples nos dá uma ação hamiltoniana e que a aplicação comomento é única a menos de soma de elementos do anulador do ideal de comutadores.

Essa seção é baseada em [1], capítulo 26 e [5].

\subsubsection{Existência da Aplicação Comomento}

Nesta seção, dada uma ação simplética $\psi: G \times M \rightarrow M$ qualquer, vamos tentar construir uma aplicação comomento para ela, adicionando hipóteses conforme necessário. Essa seção é baseada em [5].

Naturalmente, como a aplicação comomento nos dá funções Hamiltoneanas para cada um dos campos gerados pela ação infinitesimal, o primeiro requerimento natural seria que $X^{\eta}$ é campo Hamiltoneano para todo $\eta \in \mathfrak{g}$, ou seja, que a ação é fracamente Hamiltoneana. Podemos então construir uma aplicação linear $H: \mathfrak{g} \rightarrow C^{\infty}(M)$ tal que $X_{H(\eta)}=X^{\eta}$ para todo $\eta \in \mathfrak{g}$. Essa aplicação satisfaz a condição 1 da definição 2.3.4.

Agora tentaremos, a partir da aplicação $H$, construir uma aplicação comomento para $\psi$. Como o gradiante simplético é invariante por some de constantes, a idéia é encontrar $c: \mathfrak{g} \rightarrow \mathbb{R}$ de forma que $H\left(\left[\eta_{1}, \eta_{2}\right]\right)+c\left(\left[\eta_{1}, \eta_{2}\right]\right)=\left\{\eta_{1}, \eta_{2}\right\}$. Baseado nisso, o lema a seguir nos dá uma condição para que uma ação fracamente Hamiltoneana seja Hamiltoneana.

Lema 2.6.1. Sejam $(M, \omega)$ variedade simplética, $G$ grupo de Lie com álgebra de Lie $\mathfrak{g}$. Sejam também $\psi: G \times M \rightarrow M$ ação fracamente Hamiltoneana e $H: \mathfrak{g} \rightarrow C^{\infty}(M)$ aplicação linear tal que $X_{H(\eta)}=X^{\eta}$ para todo $\eta \in \mathfrak{g}$, defina $\tau: \mathfrak{g} \times \mathfrak{g} \rightarrow \mathfrak{X}(M)$ por $\tau\left(\eta_{1}, \eta_{2}\right)=\left\{H_{\eta_{1}}, H_{\eta_{2}}\right\}-H\left(\left[\eta_{1}, \eta_{2}\right]\right)$.

Então se existir $c: \mathfrak{g} \rightarrow \mathbb{R}$ tal que $c\left(\left[\eta_{1}, \eta_{2}\right]\right)=\tau\left(\eta_{1}, \eta_{2}\right)$ temos que $\psi$ é Hamiltoneana com aplicação comomento dada por $H+c$.

Demonstração. Defina $\mu^{*}=H+c$. Como o gradiente simplético é invariante por soma de constantes, temos que $X_{\mu^{*}(\eta)}=X^{\eta}$. Resa provar que $\mu^{*}$ é um homomorfismo entre álgebras de Lie. Observe que, dados $\eta_{1}, \eta_{2} \in \mathfrak{g}$

$$
\begin{aligned}
\mu^{*}\left(\left[\eta_{1}, \eta_{2}\right]\right) & =H\left(\left[\eta_{1}, \eta_{2}\right]\right)+c\left(\left[\eta_{1}, \eta_{2}\right]\right) \\
& =\left\{H\left(\eta_{1}\right), H\left(\eta_{2}\right)\right\}-\tau\left(\eta_{1}, \eta_{2}\right)+c\left(\left[\eta_{1}, \eta_{2}\right]\right) \\
& =\left\{H\left(\eta_{1}\right), H\left(\eta_{2}\right)\right\} \\
& =\left\{H\left(\eta_{1}\right)+c\left(\eta_{1}\right), H\left(\eta_{2}\right)+c\left(\eta_{2}\right)\right\} \\
& =\left\{\mu^{*}\left(\eta_{1}\right), \mu^{*}\left(\eta_{2}\right)\right\}
\end{aligned}
$$

Observação 2.6.2. Note que, estendendo as aplicações $\tau$ e $c$ do lema para formas invariantes à esquerda em $G$, i.e., definindo $\tilde{\tau}_{g}=\mathrm{d} L_{g^{-1}}^{*} \tau$ e $\tilde{c}_{g}=\mathrm{d} L_{g^{-1}}^{*} c$, a condição $c\left(\left[\eta_{1}, \eta_{2}\right]\right)=\tau\left(\eta_{1}, \eta_{2}\right)$ se torna $\mathrm{d} c=\tau$, ou seja que a forma $\tilde{\tau}$ é exata. Podemos verificar que $\tilde{\tau}$ é sempre fechada, tornando a questão de uma ação ser ou não Hamiltoneana profundamente conectada à cohomologia de $G$. O capítulo 26 de [1] discute essa relaçãção

Agora a pergunta natural é quando podemos encontrar a aplicação $c$ com as propriedades descritas no lema. Uma condição que garante isso é que $G$ seja semi-simples, o que, quando $G$ é compacto é equivalente a $[\mathfrak{g}, \mathfrak{g}]=\mathfrak{g}$. Não provaremos nenhuma das duas afirmações aqui. Alguns exemplos de grupos semi-simples incluem os grupos compactos clássicos $S U(n), S O(n)$ e $S p(n)$ e seus produtos diretos, para $n>1$. 
O grupo $U(n)$ não é semi-simples devido ao fato de que os múltiplos da identidade $S^{1} \cdot I d$, quando passados para a álgebra de Lie, correspondem a $\mathbb{R} \cdot I d$, um conjunto de matrizes que não são comutadores pois seu traço não é zero.

\subsubsection{Unicidade da Aplicação Comomento}

Proposição 2.6.3. Seja G é um grupo de Lie compacto com álgebra de Lie $\mathfrak{g}$. Se $\mu_{1}^{*}$ e $\mu_{2}^{*}$ são aplicações comomento para uma mesma ação Hamiltoneana de $G$, então $\mu_{1}^{*}-\mu_{2}^{*} \in[\mathfrak{g}, \mathfrak{g}]^{0}$

Demonstração. Para todo $\xi \in \mathfrak{g}$, como $\mu_{1}^{\xi}$ e $\mu_{2}^{\xi}$ são hamiltonianos do mesmo campo, temos que $\mu_{1}^{\xi}-\mu_{2}^{\xi}=c(\xi)$ é localmente constante.

Mas como $\mu_{1}^{\xi}$ e $\mu_{2}^{\xi}$ são homomorfismos de álgebras de Lie, $\mu_{1}^{\left[\xi_{1}, \xi_{2}\right]}=\mu_{2}^{\left[\xi_{1}, \xi_{2}\right]}$, logo $c\left(\left[\xi_{1}, \xi_{2}\right]\right)=0$, i.e., $c \in[\mathfrak{g}, \mathfrak{g}]^{0}=0$. Logo $\mu_{1}^{*}=\mu_{2}^{*}$

A proposição acima nos garante que aplicações comomento são únicas a menos de adição de um elemento anulador do ideal de comutadores $[\mathfrak{g}, \mathfrak{g}]^{0}$. Temos então o seguinte corolário

Corolário 2.6.4. Seja G é um grupo de Lie compacto com álgebra de Lie g. Se G é semi-simples, então toda ação Hamiltoneana de $G$ admite uma única aplicação comomento. 


\section{Capítulo 3}

\section{Aplicação Momento e Convexidade}

\subsection{Introdução}

Neste capítulo visamos demonstrar alguns teoremas, todos baseados na imagem da aplicação momento de uma ação Hamiltoneana de um toro. Esse capítulo é baseado em [1], [3] e [5].

O primeiro é o teorema de Atiyah-Guillemin-Sternberg, ou Teorema de convexidade, cujo enunciado é apresentado a seguir. Aqui $\mathrm{CH}\left(\lambda_{1}, \ldots, \lambda_{N}\right)$ representa o casco convexo dos pontos $\left(\lambda_{1}, \ldots, \lambda_{N}\right)$.

Teorema 3.1.1 (Convexidade). Seja $(M, \omega)$ uma variedade simplética conexa e compacta. Se $\psi$ : $T^{n} \times M \rightarrow M$ é uma ação hamiltoniana de $T^{n}$ em $M$ e $\mu: M \rightarrow \mathfrak{t}^{*}$ é uma aplicação momento para essa ação, então os pontos fixos de $\psi$ são dados por uma reunião finita de subvariedades simpléticas, conexas de $M$

$$
\operatorname{Fix}\left(T^{m}\right)=\bigcap_{\theta \in T^{n}} \operatorname{Fix}\left(\psi_{\theta}\right)=\bigcup_{i=1}^{N} C_{i}
$$

Além disso $\mu$ é constante em cada $C_{i}$ e, se $\lambda_{i}$ é o valor de $\mu$ em $C_{i}$, então a imagem de $\mu$ é o casco convexo de $\left(\lambda_{1}, \ldots, \lambda_{N}\right)$

$$
\mu(M)=\mathrm{CH}\left(\lambda_{1}, \ldots, \lambda_{N}\right)
$$

Para tal utilizaremos teoria de Morse, bem como estruturas triplas (simplética, Riemanniana e quase-complexa), que farão a ponte entre teoria de Morse e geometria simplética.

O segundo teorema é o teorema de Schur e Horn. Esse resultado é na verdade um corolário do teorema 3.1.1, aplicado no caso da ação coadjunta de $U(n)$.

Para enunciarmos esse teorema, vamos apenas introduzir algumas notações. Seja $\lambda \in \mathbb{R}^{n}$ e defina $P(\lambda)=\left\{\left(\lambda_{s(1)}, \ldots, \lambda_{s(n)}\right) \mid s \in S_{n}\right\}$, onde $S_{n}$ é o grupo das permutações de $n$ elementos, e $C(\lambda)=\mathrm{CH}(P(\lambda))$. Seja também $\mathcal{H}(n)$ o subgrupo das matrizes hermitianas de ordem $n$. O teorema de Schur e Horn pode ser enunciado da seguinte maneira.

Teorema 3.1.2 (Schur e Horn). Sejam $\lambda, d \in \mathbb{R}^{n}$. Existe $A \in \mathcal{H}(n)$ com diagonal $d$ e espectro $\lambda$ se, e só se, $d \in C(\lambda)$.

A prova deste teorema é feita encontrando uma ação hamiltoneana nas matrizes anti-hermitianas de orden $n$ (que são facilmente identificadas $\operatorname{com} \mathcal{H}(n)$ via multiplicação por $i$ ) para a qual a função que retorna a diagonal de cada matriz seja aplicação momento.

O último teorema é o teorema de Delzant. Este é um teorema de classificação de um subconjunto das variedades simpléticas. As variedades aqui classificadas são chamadas variedades tóricas simpléticas, mas as chamaremos apenas de variedades tóricas. Uma variedade tórica é um $T^{n}$-espaço Hamiltoneano $\left(M, \omega, T^{n}, \mu\right)$ tal que $M$ é $2 n$-dimensional e a ação de $T^{n}$ em $M$ é efetiva. Neste teorema utilizaremos também o conceito de politopo de Delzant, que será apropriadamente definido na seção 3.4 .

O teorema de Delzant pode ser enunciado da seguinte forma. 
Teorema 3.1.3 (Delzant). Existe uma correspondência um a um entre variedades tóricas e politopos de Delzant. Essa correspondência é dada pela imagem da aplicação momento, ou seja, a aplicação

$$
(M, \omega, \psi, \mu) \mapsto \mu(M)
$$

Esse é um teorema com uma demosntração bastante técnica e não será demonstrado aqui. Ao invés disso na seção 3.4 construiremos a variedade tórica associada a um politopo particular, dando assim uma idéia geral do esqueleto da prova.

\subsection{Teorema de Convexidade}

Aqui apresentaremos a demonstração segundo Atiyah do teorema de convexidade. Essa seção é baseada em [3], [?] e [5].

\subsubsection{Estruturas Quase-Complexas}

Dada $M$ variedade, uma estrutura quase-complexa em $M$ é uma função $J:$ T $M \rightarrow$ T $M$ tal que $J_{x}: \mathrm{T}_{x} M \rightarrow \mathrm{T}_{x} M$ é linear e $J^{2}=-\mathrm{Id}$.

Se $(M, \omega)$ é uma variedade simplética, $J$ é uma estrutura quase-complexa em $M$ e $g$ é uma métrica Riemaniana em $M$, então a tripla $(\omega, g, J)$ é dita compatível se $\omega(\cdot, \cdot)=g(J(\cdot), \cdot)$. Um fato importante, e que não será provado aqui, é que dados dois dos elementos da tripla sempre podemos obter o terceiro de modo que formem uma tripla compatível. Em particular, como toda variedade admite métrica Riemaniana, toda variedade simplética admite estrutura quase-complexa.

Vamos agora provar alguns resultados que serão úteis mais adiante.

Lema 3.2.1. Seja $M$ uma variedade e $\omega, g, J$ forma simplética, métrica e estrutura quase-complexa em $M$, respectivamente, formando uma tripla compativel. Então vale que:

(a) Se $Q$ é subvariedade de $M$ tal que $J(\mathrm{~T} Q)=\mathrm{T} Q$, então $Q$ é subvariedade simplética.

(b) Seja $f: M \rightarrow M$ suave. Se duas das afirmações abaixo são verdadeiras, a terceira também o é

- $f$ é simplética, i.e., $f^{*} \omega=\omega$

- $f$ é Riemanniana, i.e., $f^{*} g=g$

- $f$ é unitária, i.e., $\mathrm{d} f \circ J=J \circ \mathrm{d} f$

(c) Seja $f: M \rightarrow \mathbb{R}$ suave. Então $\operatorname{grad}(f)=J \circ X_{f}$

Demonstração. Para provar o item (a), lembre que $Q$ é subvariedade simplética de $M$ se, e só se, $\mathrm{T}_{p} Q$ é subespaço vetorial simplético de $\mathrm{T}_{p} M$, para todo $p \in Q$.

Suponha agora que existe $X \in \mathrm{T}_{p} Q$ tal que $\omega(X, Y)=0$, para todo $Y \in \mathrm{T}_{p} Q$. Então temos que

$$
0=\omega(X, Y)=g(J(X), Y)
$$

Mas, como $J(X) \in \mathrm{T}_{p} Q$, temos, em particular, que $g(J(X), J(X))=0$, logo $X=0$. Dai conclui-se que $\mathrm{T}_{p} Q^{\omega} \cap \mathrm{T}_{p} Q=\{0\}$, $\operatorname{logo} \mathrm{T}_{p} Q$ é subespaço simplético de $\mathrm{T}_{p} M$.

Para provar o item (b), defina as funções $j=\left(J, \operatorname{Id}_{\mathrm{T} M}\right)$ e $F=(\mathrm{d} f, \mathrm{~d} f)$. Note que a definição de compatibilidade pode ser escrita como $\omega=g \circ j$. Veja também que $f$ é unitária se, e só se, $j \circ F=F \circ j$.

Assuma primeiro que $f$ é Riemanniana e unitária. Faremos aqui um pequeno abuso com a notação de pull-back. No caso de $f$, o pull-back indicará a composição da 2 -forma com $(\mathrm{d} f, \mathrm{~d} f)$, 
enquanto que no caso de $F$ e $j$ o pull-back é simplesmente a composição das duas funções. Dessa forma $f^{*} \omega=F^{*} \omega$. Assim

$$
\begin{aligned}
f^{*} \omega & =F^{*}(g \circ j) \\
& =(g \circ j \circ F) \\
& =(g \circ F \circ j) \\
& =(F \circ j)^{*} g \\
& =j^{*}\left(F^{*} g\right) \\
& =j^{*} g \\
& =\omega
\end{aligned}
$$

O caso em que $f$ é simplética e unitária é análogo, já que $g=\omega \circ\left(-J, \operatorname{Id}_{\mathrm{T} M}\right)$

Para o caso $f$ é Riemanniana e simplética, observe que

$$
\begin{aligned}
f^{*} \omega & =f^{*}\left(j^{*} g\right) \\
& =\left(F^{*} \circ j^{*}\right) g \\
& =(j \circ F)^{*} g
\end{aligned}
$$

Mas, por outro lado

$$
\begin{aligned}
f^{*} \omega & =\omega \\
& =j^{*} g \\
& =j^{*}\left(f^{*} g\right) \\
& =(F \circ j)^{*} g
\end{aligned}
$$

Em particular, temos $(F \circ j)^{*} g-(j \circ F)^{*} g=0$. Ou seja

$$
\begin{aligned}
0 & =g(\mathrm{~d} f \circ J(X), \mathrm{d} f(Y))-g(J \circ \mathrm{d} f(X), \mathrm{d} f(Y)) \\
& =g((\mathrm{~d} f \circ J-J \circ \mathrm{d} f)(X), \mathrm{d} f(Y))
\end{aligned}
$$

para todos $X, Y \in T_{p} M$. como d $f$ é um isomorfismo, temos d $f \circ J=J \circ \mathrm{d} f$.

Para o item (c), observe apenas que, para todo $p \in M$ e $Y \in T_{p} M$

$$
\begin{aligned}
g(\operatorname{grad}(f)(p), Y) & =\mathrm{d} f_{p}(Y) \\
& =\omega\left(X_{f}(p), Y\right) \\
& =g\left(J\left(X_{f}(p)\right), Y\right)
\end{aligned}
$$

e o resultado segue pela não degenerescência de $g$.

\subsubsection{Teoria de Morse}

A Teoria de Morse, mais especificamente funções de Morse-Bott, é parte do arcabouço teórico essencial para a demonstração do Teorema de Convexidade. Antes de começarmos, no entanto, seria apropriado discutir o conceito de Hessiana, que será usado nesta seção e também na demonstração do Teorema de Convexidade.

\section{Função Hessiana}

Dada uma função $f$, o operador Hessiano associa, para cada ponto do domínio, uma função bilinear relacionada à segunda derivada de $f$. A definição usual, dada em função de uma métrica e sua conexão Riemanniana é a seguinte. 
Definição 3.2.2. Seja $M$ uma variedade m-dimensional compacta dotada de uma métrica $g$ e com conexão Riemanniana $\nabla$. Se $f: M \rightarrow \mathbb{R}$ é uma função suave, a Hessiana de $f$ no ponto $p \in M$ é dada por

$$
\left(\nabla^{2} f\right)_{p}(X, Y)=g\left(\nabla_{X} \operatorname{grad}(f), Y\right)(p)
$$

para todos $X, Y \in \mathrm{T}_{p} M$. Alternativamente, podemos definir a Hessiana como um operador linear, dado por

$$
\operatorname{Hess}(f)_{p}(X)=\nabla_{X} \operatorname{grad}(f)
$$

Observação 3.2.3. Note que as duas definições acima são equivalentes. De fato, puxando $\left(\nabla^{2} f\right)_{p}$ pelo isomorfismo canônico entre 2,0-tensores e 1,1-tensores e identificando $T_{p} M$ com seu dual via o produto interno, obtemos Hess $(f)$. Dessa forma, daqui em diante denotaremos ambas as funções por $\operatorname{Hess}(f)$, a distinção devendo ficar clara pelo contexto.

Nesta seção estaremos apenas interessados na Hessiana avaliada em pontos críticos da função. O lema seguir mostra que, em tais pontos, a Hessiana independe da métrica escolhida.

Lema 3.2.4. Se $f: M \rightarrow \mathbb{R}$ é uma função suave e p é ponto crítico de $f$, então temos que

$$
\operatorname{Hess}(f)_{p}(X, Y)=X \cdot(Y \cdot f)(p)
$$

Demonstração. Seja $e_{1}, \ldots, e_{m}$ um referencial geodésico em $p$, i.e., sejam campos $e_{i} \in \mathfrak{X}(U), \operatorname{com} U$ aberto de $M$ contendo $p$, tais que $\nabla_{X} e_{i}(p)=0, \forall X \in \mathrm{T}_{p} M$, e os vetores $e_{1}(q), \ldots, e_{m}(q)$ formem uma base ortonormal de $\mathrm{T}_{q} M$ para todo $q \in U$. Então temos que

$$
\operatorname{grad}(f)=\sum_{i=1}^{m}\left(e_{i} \cdot f\right) e_{i}
$$

Considere $X, Y \in \mathrm{T}_{p} M \operatorname{com} Y=\left(y_{1}, \ldots, y_{m}\right)$ quando escrito na base $\left(e_{1}, \ldots, e_{m}\right)$. Temos então

$$
\begin{aligned}
\operatorname{Hess}(f)_{p}(X, Y) & =g\left(\nabla_{X} \operatorname{grad}(f), Y\right)(p) \\
& =g\left(\nabla_{X}\left(\sum_{i=1}^{m}\left(e_{i} \cdot f\right) e_{i}\right), Y\right)(p) \\
& =g\left(\sum_{i=1}^{m}\left(X \cdot\left(e_{i} \cdot f\right)\right) e_{i}+\sum_{i=1}^{m}\left(e_{i} \cdot f\right) \nabla_{X} e_{i}, Y\right)(p) \\
& =g\left(\sum_{i=1}^{m}\left(X \cdot\left(e_{i} \cdot f\right)\right) e_{i}, \sum_{i=1}^{m} y_{i} e_{i}\right)(p) \\
& =\sum_{i=1}^{m}\left(X \cdot\left(e_{i} \cdot f\right)\right)(p) y_{i}(p)
\end{aligned}
$$

Por outro lado observe que

$$
\begin{aligned}
X \cdot(Y \cdot f)(p) & =\sum_{i=1}^{m}\left(X \cdot\left(y_{i} e_{i} \cdot f\right)\right)(p) \\
& =\sum_{i=1}^{m}\left(X \cdot\left(e_{i} \cdot f\right)\right)(p) y_{i}(p)+\sum_{i=1}^{m}\left(\left(X \cdot y_{i}\right) e_{i} \cdot f\right)(p) \\
& =\sum_{i=1}^{m}\left(X \cdot\left(e_{i} \cdot f\right)\right)(p) y_{i}(p)
\end{aligned}
$$

onde a última igualdade vem do fato que $e_{i} \cdot f(p)=0$, pois $p$ é ponto crítico de $f$. 
Observação 3.2.5. Note que, se $p$ é ponto crítico de $f: M \rightarrow \mathbb{R}$, então, dados $X, Y \in \mathfrak{X}(M)$

$$
[X, Y] \cdot f(p)=d f_{p}([X, Y])=0
$$

Isso implica que $X \cdot Y \cdot f(p)=Y \cdot X \cdot f(p)$ e, portanto, é uma maneira para que o leitor não familiar com técnicas de geometria Riemanniana defina a Hessiana e veja que ela é um oprador simétrico, ao menos nos pontos críticos de $f$.

\section{Funções de Morse-Bott}

Uma função $f: M \rightarrow \mathbb{R}$ é dita função de Morse-Bott se o conjunto $C$ de pontos críticos de $f$ é dado por uma união disjunta $\bigcup_{i} C_{i}$ de subvariedades conexas de $M$ e, para todo $p \in C$, se $p \in C_{j}$, então $\mathrm{T}_{p} C_{j}=\operatorname{Ker} \operatorname{Hess}(f)_{p}$. As subvariedades $C_{i}$ são chamadas subvariedades críticas de $f$.

Chamamos de indice ou $n_{-}\left(C_{j}\right)$ de $f$ na subvariedade crítica $C_{j}$ a soma das dimensões dos autoespaços associados a autovalores negativos de $\operatorname{Hess}(f)$. O coíndice ou $n_{+}\left(C_{j}\right)$ de $f$ na subvariedade crítica $C$ é o equivalente para autovalores positivos. Observe que

$$
n_{+}\left(C_{j}\right)+n_{-}\left(C_{j}\right)+\operatorname{dim} \mathrm{T}_{x} C_{j}=\operatorname{dim} M
$$

A seguir apresentaremos dois lemas que são pilares da demonstração segundo Atiyah do Teorema de Convexidade. Apresentaremos esses resultados sem demonstração por hora. As provas podem ser encontradas na seção 3.2.4. Os lemas e suas provas, exceto quando especificado o contrário, foram retirados de [3].

O primeiro resultado é apenas um resultado de teoria de Morse-Bott.

Lema 3.2.6. Se $f: M \rightarrow \mathbb{R}$ é uma função de Morse-Bott com indice e coíndice diferentes de 1 em todo ponto, então para todo valor regular $c$ de $f$ os conjuntos de nível $f^{-1}(c)$ são conexos.

Agora voltaremos nossa atenção a variedades simpléticas e a aplicação momento. Sejam $(M, \omega)$ uma variedade simplética, $\psi: T^{n} \times M \rightarrow M$ ação hamiltoniana com aplicação momento $\mu$. Usaremos aqui a identificação $\operatorname{Lie}\left(T^{n}\right) \approx \mathbb{R}^{n}$.

O lema seguinte, diz, entre outras coisas, que as componentes da aplicação momento atendem as condições do lema 3.2.6. Usaremos as notações $\operatorname{Fix}(\psi)$ para os pontos fixos de uma ação $\psi$ e $T^{\eta}$ para o subtoro fechado gerado pelo elemento $\eta$ da álgebra de Lie de $T^{m}$, mais especificamente $T^{\eta}=\overline{\{\exp (t \eta) \mid t \in \mathbb{R}\}}$.

Lema 3.2.7. Para todo $\eta \in \mathfrak{g}$, temos que $\mu^{\eta}$ é uma função de Morse-Bott com o índice e coíndice pares em todo ponto. Além disso, o conjunto de pontos críticos de $\mu^{\eta}$

$$
\operatorname{Crit}\left(\mu^{\eta}\right)=\bigcap_{\theta \in T^{\eta}} \operatorname{Fix}\left(\psi_{\theta}\right)=\operatorname{Fix}(\psi)
$$

é uma subvariedade simplética de $M$.

Em particular, dos dois lemas acima obtemos o corolário:

Corolário 3.2.8. Para todo $\eta \in \mathfrak{g}$ e todo $c \in \mathbb{R}$ valor regular de $\mu_{\eta}$, temos que $\mu_{\eta}^{-1}(c)$ é conexo.

\subsubsection{Prova do Teorema de Convexidade}

Vamos agora provar o Teorema de Atiyah-Guillemin-Steinberg. Essa demonstração é baseada em [?].

Teorema (Convexidade). Seja $(M, \omega)$ uma variedade simplética conexa e compacta. Se $\psi: T^{m} \times$ $M \rightarrow M$ é uma ação hamiltoniana de $T^{m}$ em $M$ e $\mu: M \rightarrow \mathfrak{t}^{*}$ é uma aplicação momento para essa 
ação, então os pontos fixos de $\psi$ são dados por uma reunião finita de subvariedades simpléticas, conexas $C_{i}$ de $M$

$$
\operatorname{Fix}(\psi)=\bigcap_{\theta \in T^{m}} \operatorname{Fix}\left(\psi_{\theta}\right)=\bigcup_{i=1}^{N} C_{i}
$$

Além disso $\mu$ é constante em cada $C_{i}$ e, se $\lambda_{i}$ é o valor de $\mu$ em $C_{i}$, então a imagem de $\mu$ é o casco convexo de $\left(\lambda_{1}, \ldots, \lambda_{N}\right)$

$$
\mu(M)=\mathrm{CH}\left(\lambda_{1}, \ldots, \lambda_{N}\right)
$$

Durante a prova, iremos enunciar alguns lemas, os quais serão provados mais adiante na seção 3.2.4, na tentativa de remover ao máximo resultados técnicos nessa apresentação da prova.

A prova desse teorema pode ser dividida em três passos:

Passo 1: Provar que $\mu^{-1}(c)$ é conexo para todo $c \in E$, onde $E$ é conjunto denso em $\mathfrak{t}^{*}$.

A prova é feita por indução na dimensão de $T^{m}$. Se $m=1$, temos $\mathfrak{t}^{*} \approx \mathbb{R}$ e a aplicação momento é identificada com uma de suas componentes, logo basta aplicar o corolário 3.2.8.

Seja $\psi$ ação de $T^{m}$ em $M$ e assuma que o enunciado vale para toda ação de $T^{k}$ em $M$ com $k<m$. Vamos considerar primeiro o caso em que $\psi$ não é efetiva. Para isso usaremos o lema a seguir.

Lema 3.2.9. Se $\psi: T^{m} \times M \rightarrow M$ é ação não efetiva e $\mu$ sua aplicação momento, então existem um subtoro $\tilde{T}(m-1)$-dimensional de $T^{m}$ com álgebra de Lie $\tilde{\mathfrak{t}}, \pi^{*}: \tilde{\mathfrak{t}}^{*} \rightarrow \mathfrak{t}^{*}$ aplicação linear $e$ $\tilde{\mu}: M \rightarrow \tilde{\mathfrak{t}}$ aplicação momento para $\tilde{\psi}=\left.\psi\right|_{\tilde{T} \times M}$ tais que $\mu=\pi^{*} \circ \tilde{\mu}$.

Pela hipótese de indução, $\tilde{\mu}(M)$ é convexo e, pela linearidade de $\pi^{*}, \mu(M)$ também o é. Logo podemos considerar $\psi$ efetiva.

Se $\langle\cdot, \cdot\rangle$ é produto interno e $B=\left(\eta_{1}, \ldots, \eta_{m}\right)$ é base ortonormal de $\mathfrak{t}$, escreva $\mu_{i}=\mu^{\eta_{i}}$, onde $\mu$ é uma aplicação momento para $\psi$ e defina $\nu=\left\langle\left(\mu_{1}, \ldots, \mu_{m-1}\right), \cdot\right\rangle$. O lema 2.3 .15 nos garante que $\nu$ é aplicação momento para ação do subtoro $(n-1)$-dimensional de $T^{n}$ gerado por $\eta_{1}, \ldots, \eta_{m-1}$.

Vamos aceitar o seguinte lema.

Lema 3.2.10. Sejam $M$ variedade, $V$ espaço vetorial e $B=\left(v_{1}, \ldots, v_{n}\right)$ uma base de $V$. Se $f: M \rightarrow V$ é uma aplicação suave e $\left(f_{1}, \ldots, f_{n}\right)$ são as coordenadas de $f$ na base $B$, então o conjunto $E_{f}$ dos pontos $p=\left(p_{1}, \ldots, p_{n}\right) \in V$ tais que $\left(p_{1}, \ldots, p_{k}\right)$ é valor regular de $\left(f_{1}, \ldots, f_{k}\right)$, para $0<k \leq n$ é denso em $V$.

Considere $\zeta \in E_{\mu}$. Em particular $\left(\zeta_{1}, \ldots, \zeta_{m-1}\right)$ é valor regular de $\nu$ e podemos definir $Q=$ $\nu^{-1}\left(\zeta_{1}, \ldots, \zeta_{m-1}\right)$ subvariedade de $M$ que, pela hipótese de indução, é conexo. Assim, temos a igualdade $\mu^{-1}(\zeta)=Q \cap \mu_{m}^{-1}(\zeta)=\left(\left.\mu_{m}\right|_{Q}\right)^{-1}\left(\zeta_{m}\right)$. Logo, pelo lema 3.2.6, teremos provado o passo 1 se provarmos a seguinte afirmação:

Afirmação 3.2.11. $\left.\mu_{m}\right|_{Q}$ é função de Morse-Bott com índice e coíndice diferentes de 1.

Mas observe que, pelo método de multiplicadores de Lagrange, podemos encontrar números $\theta_{1}, \ldots, \theta_{m-1} \in \mathbb{R}$ tais que

$$
\left.\mathrm{d} \mu_{m}\right|_{Q}=\left.\sum_{i=1}^{m-1} \theta_{i} \mathrm{~d} \mu_{i}\right|_{Q}
$$

e portanto se escolhermos $\theta \in \mathfrak{t}$ como o vetor cujas coordenadas na base $B$ são $\left(-\theta_{1}, \ldots,-\theta_{m-1}, 1\right)$ teremos que

$$
\left.\mathrm{d} \mu_{\theta}\right|_{Q}=\left.\mathrm{d} \mu_{m}\right|_{Q}-\left.\sum_{i=1}^{m-1} \theta_{i} \mathrm{~d} \mu_{i}\right|_{Q}
$$

Como, em $Q, \mu_{1}, \ldots \mu_{m-1}$ são constantes, temos que se $\left.\mu^{\theta}\right|_{Q}$ for função de Morse-Bott, então $\left.\mu_{m}\right|_{Q}$ também o será e ambas funções terão mesmo índice e coíndice. Logo, basta provar a afirmação 3.2.11 com $\left.\mu_{\theta}\right|_{Q}$ no lugar de $\left.\mu_{m}\right|_{Q}$.

Pelo lema 3.2.7, temos que $\mu^{\theta}$ é função de Morse-Bott com índice e coíndice pares em todo ponto. Vamos assumir mais um lema. 
Lema 3.2.12. Sejam $\mu, \nu$ e $Q$ como definidos no passo acima. Então, se $\theta \in \mathfrak{t}$ e $C$ é subvariedade crítica de $\mu_{\theta}$ passando por $x \in Q$, então $Q$ e $C$ se interseptam transversalmente, ou seja, temos que $\mathrm{T}_{x} C+\mathrm{T}_{x} Q=\mathrm{T}_{x} M$.

Uma prova detalhada será dada na seção 3.2.4. O argumento se baseia no fato de que os fluxos Hamiltoneanos de $\mu_{1}, \ldots, \mu_{m-1}, \mu_{\theta}$ comutam, pois $T^{m}$ é abeliano. Isso implica que os gradientes simpléticos $X_{\mu_{1}}, \ldots, X_{\mu_{m-1}}$ são tangentes a $C$. O fato de $T_{x} C$ ser simplético, garantido pelo lema 3.2.7, e os campos serem lineramente independentes, grantido pela ação ser efetiva, implicará que os funcionais lineares $d \mu_{i}(x): C \rightarrow \mathbb{R}$ são linearmente independentes, que, por sua vez, implicará na transversalidade.

Usando o lema 3.2.12, podemos encontrar $T_{x} C^{C} \subset T_{x} Q$ complemento de $T_{x} C$. Mas note que $T_{x} C^{C}$ também é um complemento de $T_{x}(C \cap Q)$ em $T_{x} Q$. Ou seja, as hessianas de $\mu_{\theta}$ e $\left.\mu_{\theta}\right|_{Q}$ tem mesma parte não degenerada. Isso implica que $\left.\mu_{\theta}\right|_{Q}$ é uma função de Morse-Bott com índice e coíndice pares, provando a afrimação 3.2 .11 e, portanto, o passo 1.

Passo 2: Provar que $\mu(M)$ é convexo

Para provar o passo 2, basta mostrar que, dados $v_{1}, v_{2} \in \mu(M)$, temos $t v_{1}+(1-t) v_{2} \in \mu(M)$ para todo $t \in[0,1]$.

Vamos tratar primeiro do caso em que $v_{1}, v_{2} \in \mu(M) \cap E_{\mu}$, com $E_{\mu}$ definido como no lema 3.2.10.

Fixe $\langle\cdot, \cdot\rangle$ produto interno em $\mathfrak{t}$ e denote por $\theta: \mathfrak{t} \rightarrow \mathfrak{t}^{*}$ o isomorfismo de Riesz $\theta(\eta)=\langle\eta, \cdot\rangle$. Denote também por $\langle\cdot, \cdot\rangle$ o produto interno induzido em $\mathfrak{t}^{*}$ por $\theta$.

Denote por $r^{\perp}$ o hiper-plano de $\mathfrak{t}^{*}$ perpendicular a $r$ que contém o ponto 0. Sejam $v \in \mathfrak{t}^{*}$ o ponto na interseção $r \cap r^{\perp}$ e $\pi_{r^{\perp}}: \mathfrak{t}^{*} \rightarrow r^{\perp}$ projeção ortogonal. Note que $v_{1}$ e $v_{2}$ são valores regulares de $\mu$, portanto existe $\epsilon>0$ tal que $B_{\epsilon}\left(v_{1}\right)$ e $B_{\epsilon}\left(v_{2}\right)$ estão contidos em $\mu(M)$. Temos então que $B_{\epsilon}=\pi\left(B_{\epsilon}\left(v_{1}\right)\right)=\pi\left(B_{\epsilon}\left(v_{2}\right)\right)$ é uma bola de raio $\epsilon$ e centro $v$ no hiper-plano $r^{\perp}$. Logo, exise $w \in B_{\epsilon} \cap E_{\pi \circ \mu}$ e podemos encontrar $w_{1} \in B_{\epsilon}\left(v_{1}\right)$ e $w_{2} \in B_{\epsilon}\left(v_{2}\right)$ e uma reta $\tilde{r}$ paralela a $r$ contendo $w, w_{1}, w_{2}$. Esse processo é ilustrado na figura 3.1.

Se mostrarmos que $t w_{1}+(1-t) w_{2} \in \mu(M)$ para todo $t \in[0,1]$, poderemos, como $\epsilon$ é arbitrário, construir sequências $w_{1}^{n} \rightarrow v_{1}$ e $w_{2}^{n} \rightarrow v_{2}$ com $t w_{1}^{n}+(1-t) w_{2}^{n} \in \mu(M)$, o que, junto com a compacidade de $M$, nos garante o resultado desejado.

Note agora que basta provar que $\tilde{r} \cap \mu(M)$ é conexo. Assim, como $\mu$ é contínua, é suficiente que $\mu^{-1}(\tilde{r})$ seja conexo. Ou ainda, basta mostrar que $\left(\mu \circ \pi_{\tilde{r}^{\perp}}\right)^{-1}(w)$ é conexo. Vamos mostrar que $\pi_{r \perp} \circ \mu$ é (quase) aplicação momento para a ação de um toro, e aplicaremos o passo 1 para concluir a prova.

Escreva $\tilde{\mathfrak{t}}=\theta^{-1}\left(\tilde{r}^{\perp}\right)$ e defina $\tilde{\theta}: \tilde{\mathfrak{t}} \rightarrow \tilde{\mathfrak{t}}^{*}$ isomorfismo de Riesz dado pelo produto interno $\langle\cdot, \cdot\rangle$ restrito a $\tilde{\mathfrak{t}}$. Então $\Theta_{\tilde{r}^{\perp}}=\left.\tilde{\theta} \circ \theta^{-1}\right|_{\tilde{r}^{\perp}}: \tilde{r}^{\perp} \rightarrow \tilde{\mathfrak{t}}^{*}$ é um isomorfismo entre $\tilde{r}^{\perp}$ e $\tilde{\mathfrak{t}}^{*}$.

Mas obsere que, se $i_{\tilde{\mathfrak{t}}}: \tilde{\mathfrak{t}} \rightarrow \mathfrak{t}$ é a inclusão então $i_{\tilde{\mathfrak{t}}}^{*}=\Theta_{\tilde{r}^{\perp}} \circ \pi_{\tilde{r}^{\perp}}$. Logo, se $\tilde{T}$ é o subtoro gerado por $\tilde{\mathfrak{t}}$, o lema 2.3.15 nos garante que $\Theta_{\tilde{r} \perp} \circ \pi \circ \mu$ é uma aplicação momento para a ação restrita a $\tilde{T}$.

Além disso, como $\Theta_{\tilde{r}^{\perp}}$ é isomorfismo, é fácil mostrar que $E_{\Theta_{\tilde{r}^{\perp}} \circ \pi_{\tilde{r} \perp}}=\Theta_{\tilde{r}^{\perp}}\left(E_{\pi_{\tilde{r} \perp}}\right)$. Basta ver que $x \in \tilde{\mathfrak{t}}^{*}$ é valor regular de $\Theta_{\tilde{r}^{\perp}} \circ \pi_{\tilde{r}^{\perp}}$ se, e só se, $\Theta_{\tilde{r}^{\perp}}(x)$ é valor regular de $\pi_{\tilde{r}^{\perp}}$ e que o mesmo vale quando consideramos apenas as primeiras $k$ componentes de cada função e ponto. Logo, se $\tilde{w}=\Theta_{\tilde{r}^{\perp}}(w)$, temos que $\tilde{w} \in E_{\Theta_{\tilde{r}^{\perp}} \circ \pi_{\tilde{r} \perp}}$ e, pelo passo 1 ,

$$
\left(\Theta_{\tilde{r}^{\perp}} \circ \pi \circ \mu\right)^{-1}(\tilde{w})=(\pi \circ \mu)^{-1}(w)
$$

é conexo.

Considere agora $v_{1}, v_{2} \in \mu(M)$ quaisquer. Para todo $\epsilon>0$ podemos encontrar $w_{1}, w_{2} \in \mu(M) \cap$ $E_{\mu}$ com $w_{1} \in B_{\epsilon}\left(v_{1}\right)$ e $w_{2} \in B_{\epsilon}\left(v_{2}\right)$. Logo existem sequências $w_{1}^{n} \rightarrow v_{1}$ e $w_{2}^{n} \rightarrow v_{2}$ em $\mu(M) \cap E_{\mu}$. Pelo caso anterior sabemos que $t w_{1}^{n}+(1-t) w_{2}^{n} \in \mu(M)$ para todo $t \in[0,1]$ e, como $\mu(M)$ é compacto, temo que $t v_{1}+(1-t) v_{2} \in \mu(M)$.

Passo 3: Provar que $\mu(M)=C H\left(\lambda_{1}, \ldots, \lambda_{N}\right)$

Pelo lema 3.2.7, temos que $\operatorname{Fix}\left(T^{m}\right)=\bigcup_{i=1}^{n} C_{i}$, onde $C_{i}$ são as subvariedades críticas de $\mu$. Logo $\mu$ é constante em cada $C_{i}$, e podemos escolher $\lambda_{i} \in \mathfrak{t}^{*}$ tal que $\mu\left(C_{i}\right)=\left\{\lambda_{i}\right\}$ para todo $i \in\{1, \ldots, n\}$. 


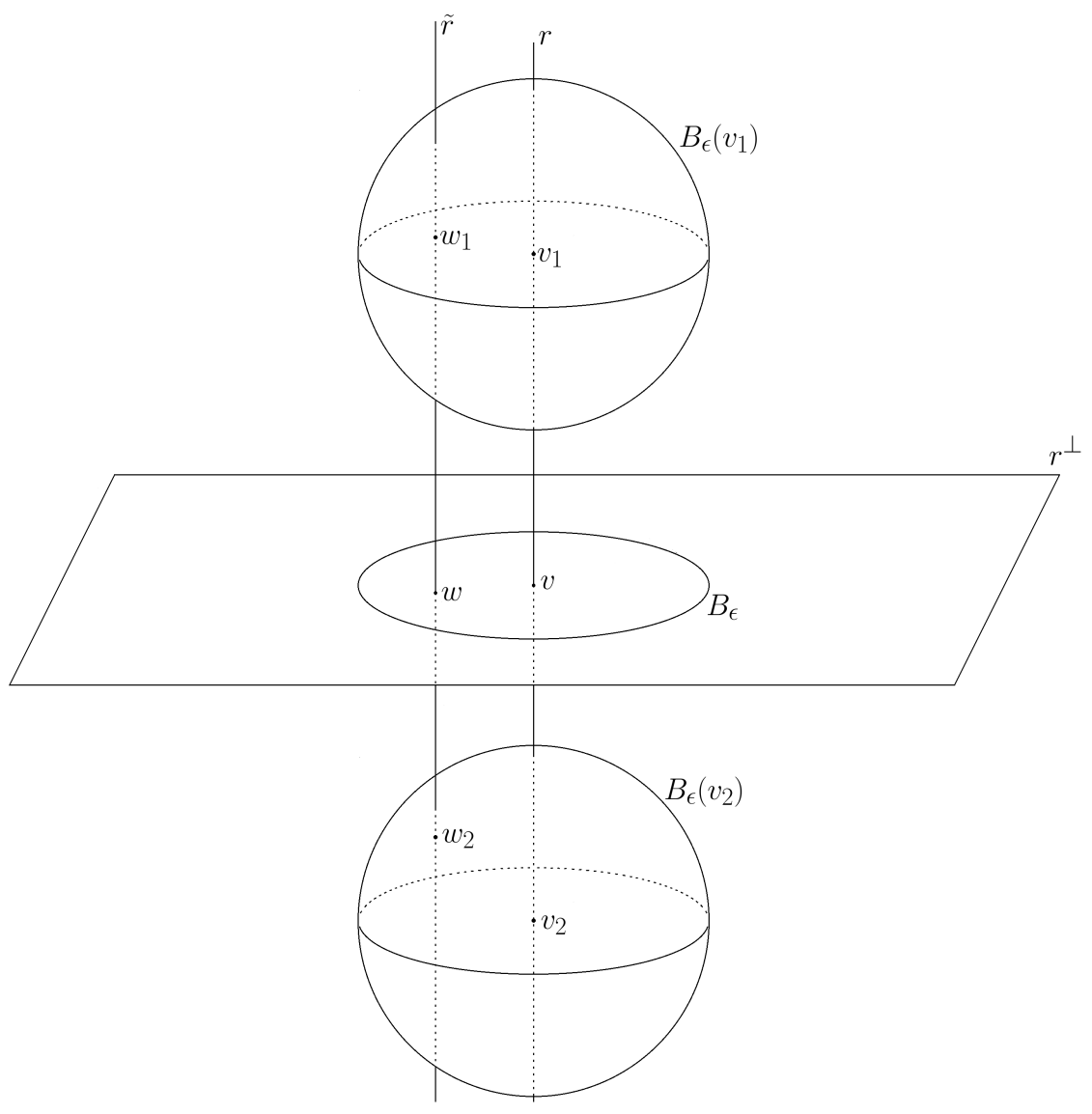

Figura 3.1: Aproximando a reta $r$ por uma reta $\tilde{r}$ que intercepta $r^{\perp}$ em um ponto de $E_{\pi \circ \mu}$ 
Como $\mu(M)$ é conexo temos $\mathrm{CH}\left(\lambda_{1}, \ldots, \lambda_{N}\right) \subset \mu(M)$. Para provar a outra inclusão considere o seguinte lema.

Lema 3.2.13. Se $V$ é um espaço vetorial e $v_{1}, \ldots, v_{n}, v \in V$, são vetores tais que $v \notin \mathrm{CH}\left(v_{1}, \ldots, v_{n}\right)$, então existe $w \in V^{*}$ tal que $\left.\langle w, v\rangle\right\rangle\left\langle w, v_{i}\right\rangle$ para todo $i \in\{1, \ldots, n\}$.

Seja $\xi \in \mathfrak{t} \backslash \operatorname{CH}\left(\lambda_{1}, \ldots, \lambda_{N}\right)$. Então pelo lema 3.2 .13 temos que existe $\eta \in \mathfrak{t}^{*}$ tal que vale $\langle\eta, \xi\rangle>\left\langle\eta, \lambda_{i}\right\rangle$ para todo $i \in\{1, \ldots, n\}$.

Observe que $\mathrm{d}\left(\mu_{\eta}\right)_{p}=0$ se, e só se, $p \in \bigcup_{i=1}^{n} C_{i}$. Logo $\mu_{\eta}=\langle\eta, \mu(\cdot)\rangle$ atinge seu máximo em algum $\operatorname{dos} C_{i}$. Mas, temos que

$$
\langle\eta, \xi\rangle>\sup _{i}\left\langle\eta, \lambda_{i}\right\rangle=\sup _{p \in M}\langle\eta, \mu(p)\rangle
$$

Logo temos que $\xi \notin \mu(M)$, o que prova o passo 3 e o teorema.

\subsubsection{Prova dos Lemas}

Nesta seção vamos provar os resultados que ficamos devendo nas seções 3.2.3 e 3.2.2. O primeiro é o lema 3.2.9, que foi baseado em [10].

Lema (3.2.9). Se $\psi: T^{m} \times M \rightarrow M$ é ação não efetiva e $\mu$ sua aplicação momento, então existem um subtoro $\tilde{T}(m-1)$-dimensional de $T^{m}$ com álgebra de Lie $\tilde{\mathfrak{t}}, \pi^{*}: \tilde{\mathfrak{t}}^{*} \rightarrow \mathfrak{t}^{*}$ função suave e $\tilde{\mu}: M \rightarrow \tilde{\mathfrak{t}}$ aplicação momento para $\tilde{\psi}=\left.\psi\right|_{\tilde{T} \times M}$ tais que $\mu=\pi^{*} \circ \tilde{\mu}$.

Demonstração. Se $\psi$ não é efetiva, existe $g \in T^{m}$ tal que $\psi_{g}=\operatorname{Id}_{M}$. Isso implica que $\psi_{t \theta}=\operatorname{Id}_{M}$ para todo $t \in \mathbb{R}$. Logo, se $\tilde{\eta} \in \mathfrak{t}$ é tal que $\exp (\tilde{\eta})=\theta$, temos $X_{\mu^{\tilde{\eta}}}=X^{\tilde{\eta}}=0$.

Fixando um produto interno $\langle\cdot, \cdot\rangle$, defina a sub-álgebra $\tilde{\mathfrak{t}}=\{\eta \in \mathfrak{t} \mid\langle\eta, \tilde{\eta}\rangle=0\}$ e $\tilde{T}$ o subgrupo gerado por ela. Pelo lema 2.3 .15 , temos que a ação $\tilde{\psi}=\left.\psi\right|_{\tilde{T} \times M}$ é hamiltoneana com aplicação momento $\tilde{\mu}=i_{\tilde{\mathfrak{t}}}^{*} \circ \mu$, onde $i_{\tilde{\mathfrak{t}}}: \tilde{\mathfrak{t}} \rightarrow \mathfrak{t}$ é a inclusão.

Se considerarmos $\pi: \mathfrak{t} \rightarrow \tilde{\mathfrak{t}}$ a projeção ortogonal sobre $\tilde{\mathfrak{t}}$, temos que para todo $\eta \in \mathfrak{t}$ vale a decomposição $\eta=\pi(\eta)+\langle\eta, \tilde{\eta}\rangle \tilde{\eta}$. Logo

$$
\begin{aligned}
\pi^{*} \circ \tilde{\mu}(p)(\eta) & =\left(i_{\tilde{\mathfrak{t}}} \circ \pi\right)^{*} \circ \mu(\eta) \\
& =\mu(p)(\pi(\eta)) \\
& =\mu(p)(\eta)-\mu(p)(\langle\eta, \tilde{\eta}\rangle \tilde{\eta}) \\
& =\mu(p)(\eta)
\end{aligned}
$$

concluindo a prova.

Lema (3.2.10). Sejam $M$ variedade, $V$ espaço vetorial e $B=\left(v_{1}, \ldots, v_{n}\right)$ uma base de $V$. Se $f: M \rightarrow V$ é uma função suave $e\left(f_{1}, \ldots, f_{n}\right)$ são as coordenadas de $f$ na base $B$, então o conjunto $E_{f}$ dos pontos $p=\left(p_{1}, \ldots, p_{n}\right) \in V$ tais que $\left(p_{1}, \ldots, p_{k}\right)$ é valor regular de $\left(f_{1}, \ldots, f_{k}\right)$, para $0<k \leq n$ é denso em $V$.

Demonstração. Para efeito de simplificar a notação, vamos usar a identificação $V \approx \mathbb{R}^{n}$ que leva $B$ na base canônica. Seja, para cada $i \in\{1, \ldots, n\}$, o conjunto $\mathfrak{R}_{\left(f_{1}, \ldots, f_{i}\right)}$ dos pontos regulares de $\left(f_{1}, \ldots, f_{i}\right): M \rightarrow \mathbb{R}^{i}$ e a função $\pi_{i}: \mathbb{R}^{n} \rightarrow \mathbb{R}^{i}$ a projeção das $i$ primeiras coordenadas. Então temos que

$$
E_{f}=\bigcap_{i=1}^{n} \pi_{i}^{-1}\left(\Re_{\left(f_{1}, \ldots, f_{i}\right)}\right)
$$

Vamos primeiro mostrar que $\pi_{i}^{-1}\left(\mathfrak{R}_{\left(f_{1}, \ldots, f_{i}\right)}\right)$ é aberto e denso em $\mathbb{R}^{n}$. Pelo teorema de Sard, $\mathfrak{R}_{\left(f_{1}, \ldots, f_{i}\right)}$ é aberto e denso, e como $\pi_{i}$ é contínua, temos $\pi_{i}^{-1}\left(\mathfrak{R}_{\left(f_{1}, \ldots, f_{i}\right)}\right)$ aberto. Para provar a densidade, considere $U \subset \mathbb{R}^{n}$ aberto. Como $\pi_{i}$ é aplicação aberta $\pi_{i}(U)$ é aberto de $\mathbb{R}^{i}$ e, portanto 
$\pi_{i}(U) \cap \Re_{\left(f_{1}, \ldots, f_{i}\right)}$ é não vazio. Como, $\pi_{i}$ é sobrejetora e contínua temos que o conjunto $\pi_{i}^{-1}\left(\pi_{i}(U) \cap\right.$ $\left.\mathfrak{R}_{\left(f_{1}, \ldots, f_{i}\right)}\right)=\pi_{i}^{-1}\left(\pi_{i}(U)\right) \cap \pi_{i}^{-1}\left(\mathfrak{R}_{\left(f_{1}, \ldots, f_{i}\right)}\right)$ é não vazio, já que $\pi_{i}^{-1}\left(\pi_{i}(U)\right)$ é aberto, provando a densidade.

Logo basta provar a seguinte afirmação

Afirmação. Se $X$ é um espaço topológico e $U, V \subset X$ são abertos e densos em $X$, então $U \cap V$ é aberto de denso em $X$.

$U \cap V$ é claramente aberto, já que $U$ e $V$ são abertos. Para provar que $U \cap V$ é denso em $X$, considere $\mathcal{O} \subset X$ aberto. Como $U$ é denso em $X$, existe $u \in U \cap \mathcal{O}$. Mas, como $U \cap \mathcal{O}$ é aberto não vazio e $V$ é denso em $X$, existe $v \in V \cap U \cap \mathcal{O}$. Isso conclui a prova.

Lema (3.2.13). Se $V$ é um espaço vetorial e $v_{1}, \ldots, v_{n}, v \in V$ são tais que $v \notin \mathrm{CH}\left(v_{1}, \ldots, v_{n}\right)$, então existe $w \in V^{*}$ tal que $\langle w, v\rangle>\left\langle w, v_{i}\right\rangle$ para todo $i \in\{1, \ldots, n\}$.

Demonstração. $\mathrm{CH}\left(v_{1}, \ldots, v_{n}\right)$ é um politopo, logo podemos escolher vetores $w_{1}, \ldots, w_{k} \in V^{*} \mathrm{e}$ constantes $\lambda_{1}, \ldots, \lambda_{k} \in \mathbb{R}$ tais que

$$
\mathrm{CH}\left(v_{1}, \ldots, v_{n}\right)=\left\{u \in V \mid\left\langle u, w_{i}\right\rangle \leq \lambda_{i}, i \in\{1, \ldots, k\}\right\}
$$

Como $v \notin \mathrm{CH}\left(v_{1}, \ldots, v_{n}\right)$, temos que existe $j \in\{1, \ldots, k\}$ tal que $\left\langle v, w_{j}\right\rangle>\lambda_{j}$. Mas como $v_{1}, \ldots, v_{n}$ estão em $\mathrm{CH}\left(v_{1}, \ldots, v_{n}\right)$, temos que

$$
\left\langle v, w_{j}\right\rangle>\lambda_{j} \geq\left\langle v_{i}, w_{j}\right\rangle
$$

Lema (3.2.12). Sejam $\mu, \nu$ e $Q$ como definidos no passo 1 do teorema. Ou seja, $\mu=\left(\mu_{1}, \ldots, \mu_{n}\right)$ : $M \rightarrow \mathfrak{t}^{*}$ é aplicação momento para uma ação $\psi: T^{m} \times M \rightarrow M, \nu=\left(\mu_{1}, \ldots, \mu_{n-1}\right)$ e, se $\zeta \in E_{\mu}$ defina $Q=\nu^{-1}\left(\zeta_{1}, \ldots, \zeta_{n-1}\right)$. Então, se $\theta \in \mathfrak{t}$ e $C$ é subvariedade crítica de $\mu_{\theta}$ passando por $x \in Q$, então $Q$ e $C$ se interseptam transversalmente, ou seja, temos que $\mathrm{T}_{x} C+\mathrm{T}_{x} Q=\mathrm{T}_{x} M$.

Demonstração. Observe que se provarmos que $\left(\mathrm{T}_{x} Q\right)^{\perp} \subset \mathrm{T}_{x} C$ teremos o resultado desejado. Mas como os funcionais $\left(\mathrm{d} \mu_{i}\right)_{x}: \mathrm{T}_{x} M \rightarrow \mathbb{R}$ geram $\mathrm{T}_{x} Q^{\circ} \approx \mathrm{T}_{x} Q^{\perp}$, basta provar que esses funcionais continuam linearmente independentes quando restritos ao subespaço $\mathrm{T}_{x} C$.

Para isso vamos vamos mostrar que os fluxos hamiltoneanos de $T^{m}$ comutam, ou seja, os fluxos associados aos gradientes simpléticos $X_{\mu_{Y}}$ comutam para todo $Y \in \mathfrak{t}$.

De fato, sejam $Y_{1}, Y_{2} \in \mathfrak{t}$. Se $\varphi^{1}$ é o fluxo de $X_{\mu_{Y_{1}}}$, então para cada $p \in M, \varphi_{(\cdot)}^{1}(p)$ é um subgrupo a 1-parâmetro em $\left.M \operatorname{com} \frac{\mathrm{d}}{\mathrm{d} t} \varphi_{t}^{1}(p)\right|_{t=0}=X_{\mu_{Y_{1}}}(p)=X^{Y_{1}}(p)$. Mas $\psi_{\exp \left(t Y_{1}\right)}(p)$, também satisfaz a esses requisistos. Logo, pelo teorema de existência e unicidade de EDOs, os dois grupos a 1-parâmetro coincidem. O análogo vale para $X_{\mu_{Y_{2}}}$ e seu fluxo $\varphi^{2}$. Então

$$
\varphi_{t}^{1} \varphi_{s}^{2}(p)=\psi_{\exp \left(t Y_{1}\right)} \psi_{\exp \left(s Y_{2}\right)}(p)=\psi_{\exp \left(s Y_{2}\right)} \psi_{\exp \left(t Y_{1}\right)}(p)=\varphi_{s}^{2} \varphi_{t}^{1}(p)
$$

onde a segunda igualdade vem de $T^{m}$ ser abeliano.

Logo, os fluxos de $X_{\mu_{\theta}}$ e $X_{i}$ comutam, com $1 \leq i \leq n$. Mas como $C$ é subvarieda crítica de $\mu_{\theta}$, o fluxo $\varphi_{(\cdot)(p)}^{\theta}$ de $X_{\mu_{\theta}}$ é constante para todo $p \in C$, forçando o fluxo de $X_{i}$ a preservar $C$. Mas isso implica que $X_{i}(p) \in T_{x} C$ para todo $p \in C$.

Lembre-se que $X_{1}, \ldots, X_{n-1}$ são linearmente independentes por hipótese. Logo, dados $\lambda_{1}, \ldots, \lambda_{m-1} \in$ $\mathbb{R}$ não todos nulos, temos que $\sum_{i=0}^{m-1} \lambda_{i} X_{i} \neq 0$. Mas o lema 3.2.7 nos diz que $C$ é subvariedade simplética, $\operatorname{logo} \mathrm{T}_{x} C$ é subespaço simplético, e existe $\eta$ tal que

$$
\omega\left(\sum_{i=0}^{m-1} \lambda_{i} X_{i}, \eta\right) \neq 0
$$

Mas isso é equivalente a 


$$
\sum_{i=0}^{m-1} \lambda_{i}\left(\mathrm{~d} \mu_{i}\right)_{x} \eta \neq 0
$$

Logo os funcionais $\left(\mathrm{d} \mu_{1}\right)_{x}, \ldots,\left(\mathrm{d} \mu_{m-1}\right)_{x}$ são linearmente independents, concluindo a prova.

Vamos agora provar os lemas da seção 3.2.2, começando pelo lema 3.2.6, adaptando as demonstrações apresentadas em [3] e [5]. Antes de prová-lo, no entanto, precisamos definir um pequeno arcabouço teórico.

Lembrando das definições de função de Morse-Bott, índice e coíndice, podemos afirmar que se $f: M \rightarrow \mathbb{R}$ é de Morse-Bott, $p \in \operatorname{Crit}(f)$ e $C$ subvariedade crítica passando por $p$, então

$$
T_{p} M=T_{p} C \oplus E_{p}^{+} \oplus E_{p}^{-}
$$

onde $E_{p}^{+}$é a soma direta dos autoespaços associado a autovalores positivos de $\operatorname{Hess}(f)_{p}$ e $E_{p}^{-}$o equivalente para os negativos.

Definição 3.2.14. Sejam $f: M \rightarrow \mathbb{R}$ função de Morse-Bott, $\phi$ o fluxo associado ao campo $-\operatorname{grad}(f), p \in \operatorname{Crit}(f)$ e $C$ subvariedade crítica contendo por $p$. Então definimos o fibrado índice

$$
W^{-}(C)=\left\{x \in M \mid \lim _{t \rightarrow-\infty} \phi_{t}(x) \in C\right\}
$$

e, analogamente, o fibrado coíndice

$$
W^{+}(C)=\left\{x \in M \mid \lim _{t \rightarrow \infty} \phi_{t}(x) \in C\right\}
$$

Agora vamos apresentar algumas afirmações que serão úteis na prova do lema, mas que não serão provadas.

Afirmação 3.2.15. Se $\left\{C_{i}\right\}_{i}$ é o conjunto das subvariedades críticas de $f$, temos que $\bigcup_{i} W^{-}\left(C_{i}\right)=$ $\bigcup_{i} W^{+}\left(C_{i}\right)=M$.

Afirmação 3.2.16. Sejam $M$ variedade compacta, $D_{1}, D_{2}$ subvariedades conexas e $f: M \rightarrow \mathbb{R}$ função suave. Chame $C_{D_{1}}^{D_{2}}=\left\{\gamma:[0,1] \rightarrow M \mid \gamma(0) \in D_{1}, \gamma(1) \in D_{2}\right\}$. Então o ponto $k$ definido por

$$
k=\min _{\gamma \in C_{D_{1}}^{D_{2}}} \max _{t \in[0,1]} f(\gamma(t))
$$

é valor critico de $f$ e Hess $(f)$ tem ao menos um auto-valor negativo

Afirmação 3.2.17. Sejam $M$ variedade, $f: M \rightarrow \mathbb{R}$ função de Morse-Bott com índice maior que 1 e $\alpha:[0,1] \rightarrow M$ curva em $M$. Seja $y \in M$ valor crítico de $f$ e assuma os seguintes fatos:

- $f(\alpha(0))<y, f(\alpha(1))<y$

- Existe $\epsilon>0$ tal que $(y, y+\epsilon)$ não contém pontos críticos de $f$

- $\max _{t \in[0,1]} f(\alpha(t))<y+\epsilon$

Então existe uma curva $\tilde{\alpha}$ homotópica a a tal que $\max _{t \in[0,1]} f(\tilde{\alpha}(t))<y$.

Afirmação 3.2.18. Sejam $f: M \rightarrow \mathbb{R}$ função de Morse-Bott, $C$ subvariedade crítica de $f$ com $f(C)=c$ e $\operatorname{codim}(C) \geq 2$ e $\phi$ o fluxo associado $a-\operatorname{grad}(f)$. Se $k \in f(M)$ é tal que $k>c$ e $(c, k]$ não contém, pontos críticos de $f$, considere $x_{0}, x_{1} \in f^{-1}(k) \cap W^{+}(C)$ e faça $y_{0}=\lim _{t \rightarrow-\infty} \phi_{t}\left(x_{0}\right) e$ $y_{1}=\lim _{t \rightarrow-\infty} \phi_{t}\left(x_{1}\right)$. Então se existe curva em $C$ ligando $y_{0}$ a $y_{1}$, existe curva em $f^{-1}(k)$ ligando $x_{0} a x_{1}$. 
Afirmação 3.2.19. Sejam $f: M \rightarrow \mathbb{R}$ função de Morse-Bott e $\left\{C_{i}\right\}_{i}$ o conjunto das subvariedades críticas de $f$ assoicadas ao menor valor crítico de $f$. Então, para todo $c \in f(M), W^{+}\left(C_{i}\right)$ intercepta todas as componentes conexas de $f^{-1}(c)$.

Afirmação 3.2.20. Sejam $f: M \rightarrow \mathbb{R}$ função de Morse-Bott, $C_{0}, C$ subvariedades críticas de $f$ associadas aos valores críticos $c_{0}$ e c respectivamente, onde $c_{0}=\min (f)$ e sejam $\epsilon_{1}, \epsilon_{2}>0$ tais que c é o único valor crítico de $f$ em $\left(c-\epsilon_{1}, c+\epsilon_{2}\right)$. Se $k \in\left(c, c+\epsilon_{2}\right), \tilde{k} \in\left(c-\epsilon_{1}, c\right) e$ $x_{0}, x_{1} \in f^{-1}(k) \cap W^{+}\left(C_{0}\right)$, defina $y_{0}, y_{1} \in f^{-1}(\tilde{k})$ com a interseção dos fluxos de $x_{0}$ e $x_{1}$ com o nível $f^{-1}(\tilde{k})$. Então, se existe caminho em $f^{-1}(\tilde{k})$ ligando $y_{0}$ a $y_{1}$, temos que existe caminho em $f^{-1}(k)$ ligando $x_{0}$ a $x_{1}$.

Agora vamos finalmente provar o lema 3.2.6. Seu enunciado a lembrar é

Lema (3.2.6). Se $f: M \rightarrow \mathbb{R}$ é uma função de Morse-Bott com indice e coíndice diferentes de 1 em todo ponto, então para todo valor regular $c$ de $f$ os conjuntos de nível $f^{-1}(c)$ são conexos.

Demonstração. Vamos primeiro provar que o conjunto $\operatorname{Min}(f)$ dos mínimos globais de $f$ é conexo. Assuma por absurdo, que $\operatorname{Min}(f)$ tem duas componentes conexas $D_{1}$ e $D_{2}$. Então o ponto $k$ definido por

$$
k=\min _{\gamma \in C_{D_{1}}^{D_{2}}} \max _{t \in[0,1]} f(\gamma(t))
$$

é valor critico de $f$ com índice positivo, pela afirmação 3.2.16. Por hipóstese tem índice maior que 1. Seja $\gamma_{0} \in C_{D_{1}}^{D_{2}}$ tal que $\max _{t \in[0,1]} f(\gamma(t))=k$. Pela afirmação 3.2.17, podemos encontrar $\tilde{\gamma} \in C_{D_{1}}^{D_{2}}$ tal que $\tilde{\gamma}(t)<k$, para todo $t \in[0,1]$. Mas pela construção de $k$, tal $\tilde{\gamma}$ não pode existir. Logo Min $(f)$ é conexo. Isso implica que $\operatorname{Min}(f)$ é subvariedade crítica de $f$. Com isto, a afirmação 3.2 .18 nos garante que se $x_{0}, x_{1} \in W^{+}(\operatorname{Min}(f))$ estão no mesmo nível, temos que existe um caminho dentro deste nível conectando $x_{0}$ a $x_{1}$.

Sejam $c_{0}<\ldots<c_{N}$ os valores críticos de $f$. Vamos mostrar agora, que $f^{-1}(c)$ é conexo para todo $c \in\left(c_{0}, c_{1}\right)$. Sejam $x_{0}, x_{1} \in f^{-1}(c)$. Lembre que, pela afirmação 3.2 .15 todo ponto de $M$ pertence ao fibrado índice de alguma subvariedade crítica de $f$. Mas como $f\left(\phi_{t}(x)\right)<f(x)$ se $t<0$ e $x \in M$, onde $\phi$ é o fluxo gradiente e $\operatorname{Min}(f)=f^{-1}\left(c_{0}\right)$ é a única subvariedade crítica associada a um valor menor que $c$, temos que $x_{0}, x_{1} \in W^{+}(\operatorname{Min}(f))$. Portanto, existe curva ligando $x_{0}$ a $x_{1}$, provando a conexidade de $f^{-1}(c)$.

Vamos agora mostrar por indução que $f^{-1}(c)$ é conexo para todo $c$ valor regular de $f$. Suponha que o resultado esteja provado para todo valor regular $c<c_{j}$. Sejam $x_{0}, x_{1} \in f^{-1}\left(c_{j}+\epsilon\right)$. Pela afirmação 3.2.19, existem $x_{0}^{\prime}, x_{1}^{\prime} \in W^{+}(\operatorname{Min}(f))$ tais que $x_{0}$ e $x_{0}^{\prime}$ estão na mesma componente conexa de $f^{-1}(c)$, assim como $x_{1}$ e $x_{1}^{\prime}$. Mas, pela hipótese de indução, $f^{-1}(k)$ é conexo para todo $k$ valor regular de $f$ com $k<c_{j}$. Em conjunto com a afirmação 3.2.20, temos que existe um caminho ligando $x_{0}^{\prime}$ e $x_{1}^{\prime}$, o que nos dá um caminho ligando $x_{0}$ a $x_{1}$. Logo $f^{-1}(c)$ é conexo.

Vamos agora provar o segundo lema da seção. Os resultados daqui foram retirados de [3]. Vamos primeiro provar um resultado que vai nos auxiliar na prova do lema 3.2.7.

Lema 3.2.21. Seja $\psi$ uma ação simplética do toro $T^{m}$ em uma variedade simplética $M$. Se $G \subset T^{m}$ é um subgrupo, então o conjunto dos pontos fixos de $G$

$$
\operatorname{Fix}(\psi)=\bigcap_{\theta \in G} \operatorname{Fix}\left(\psi_{\theta}\right)
$$

é uma suvariedade simplética de $M$.

Demonstração. Seja $\Psi_{\theta}^{p}=\left(\mathrm{d} \psi_{\theta}\right)_{p}: \mathrm{T}_{p} M \rightarrow \mathrm{T}_{\psi_{\theta}(p)} M$. Note que se $p \in \operatorname{Fix}(\psi), \Psi^{p}=\Psi_{(\cdot)}^{p}$ define uma ação de $G$ em $\mathrm{T}_{p} M$. Como $T^{m}$ é compacto, existe uma métrica $g$ em $M$ tal que $\psi$ é ação isométrica. Considere $\exp _{p}: \mathrm{T}_{p} M \rightarrow M$ a exponencial Riemanniana segundo $g$. Se $\theta \in G$ e $\eta \in \mathfrak{g}$, 
então, como $\psi_{\theta}$ é isometria, temos que $\psi_{\theta}\left(\exp _{p}(\eta)\right)$ é geodésica com velocidade $\Psi_{\theta}^{p}(\eta)$. Logo, temos que

$$
\exp _{p} \circ \Psi_{\theta}^{p}=\psi_{\theta} \circ \exp _{p}
$$

Logo, numa vizinhança de $p, \theta$ é ponto fixo de $G$ se, e só se, ele é a exponencial de algum ponto fixo de $\Psi^{p}$. Além disso, como $\Psi_{\theta}^{p}$ é linear, se $\eta \in T_{p} M$ é ponto fixo, então $t \eta$ também o é, para todo $t \in \mathbb{R}$, e $\exp _{p}(t \eta)$ é ponto fixo de $\psi_{\theta}$ para $t$ pequeno. Logo, $v \in \mathrm{T}_{p} \operatorname{Fix}(G)$ se, e só se existe $\eta$ ponto fixo de $\Psi^{p}$ tal que

$$
v=\left.\frac{\mathrm{d}}{\mathrm{d} t} \exp _{p}(t \eta)\right|_{t=0}=\eta
$$

Logo, temos que

$$
\mathrm{T}_{p} \operatorname{Fix}(\psi)=\bigcap_{\theta \in G} \operatorname{Ker}\left(\Psi_{\theta}^{p}-\mathrm{Id}\right)
$$

Mas considere $J: \mathrm{T} M \rightarrow \mathrm{T} M$ estrutura quase-complexa tal que $g\left((\cdot, J(\cdot))=\omega(\cdot, \cdot)\right.$. Como $\Psi^{p}$ é simplética segundo $\omega_{p}$ e Reimanniana segundo $g_{p}$, pelo item (b) do lema 3.2.1, $\Psi^{p}$ e $J_{p}$ comutam. Mas isso implica que o auto-espaço de $\Psi_{p}$ associado a 1 é preservado por $J_{x}$, e pelo item (a) do lema 3.2.1, é simplético. Isso conclui a prova.

Finalmente, apresentamos a prova do lema 3.2.7

Lema (3.2.7). Para todo $\eta \in \mathfrak{g}$, temos que $\mu^{\eta}$ é uma função de Morse-Bott com índice e coíndice pares em todo ponto. Além disso, o conjunto de pontos críticos de $\mu^{\eta}$

$$
\operatorname{Crit}\left(\mu^{\eta}\right)=\bigcap_{\theta \in T^{\eta}} \operatorname{Fix}\left(\psi_{\theta}\right)=\operatorname{Fix}\left(T^{\eta}\right)
$$

é uma subvariedade simplética de $M$.

Demonstração. Note que os pontos críticos de $\mu^{\eta}$ equivalem a pontos fixos pela ação de qualquer elemento de $T^{m}$ do conjunto $\{\exp (t \eta) \mid t \in \mathbb{R}\}$. Como a ação é continua, esses pontos são fixos também pelo fecho do conjunto, que é $T^{\eta}$. Logo, temos

$$
\operatorname{Crit}\left(\mu^{\eta}\right)=\operatorname{Fix}\left(T^{\eta}\right)
$$

que, pelo lema 3.2.21, é uma subvariedade simplética.

Observe agora que, se $p \in M$ é ponto fixo de $T^{\eta}$ e $\nabla$ é uma conexão riemmaniana associada a uma métrica em relação à qual a ação pe isométrica, então podemos definir o campo linear $\nabla_{(\cdot)} X_{\mu \eta}(p): \mathrm{T}_{p} M \rightarrow \mathrm{T}_{p} M$. Note que este campo linear está bem definido pois $X_{\mu^{\eta}}=X^{\eta}$. Pelo item (c) do lema 3.2.1, temos

$$
\begin{aligned}
\nabla_{(\cdot)} X_{\mu^{\eta}}(p) & =\nabla_{(\cdot)}\left(J \circ \operatorname{grad}\left(\mu^{\eta}\right)\right)(p) \\
& =J_{p} \nabla_{(\cdot)} \operatorname{grad}\left(\mu^{\eta}\right)(p) \\
& =J_{p} \operatorname{Hess}\left(\mu^{\eta}\right)_{p}
\end{aligned}
$$

A igualdade $\nabla \circ J=J \circ \nabla$ pode ser verificada pelo leito no caro euclidiano em que $\nabla$ é o operador diferencial d. Para provar o caso geral podemos usar a representação isotrópica e recair no caso euclidiano.

Entretanto, se considerarmos $T_{p} M$ uma variedade Riemanniana com métrica $g_{p}$ e exp a exponencial Riemanniana associada, temos que, para cada vetor $v \in \mathrm{T}_{p} M$ o campo $\nabla_{(\cdot)} X_{\mu \eta}(p)$ gera o grupo a 1-parâmetro $\mathrm{d} \psi_{\exp (t \eta)} v$. Mas este então coincide com o grupo a 1-parâmetro $\exp \left(-t J_{p} \mathrm{Hess}_{p} v\right)$. 
Logo os pontos fixos de $\mathrm{d} \psi_{\exp (t \eta)}$ correspondem a elementos do kernel de Hess $\left(\mu^{\eta}\right)$. Ou seja

$$
\begin{aligned}
\operatorname{Ker} \operatorname{Hess}\left(\mu^{\eta}\right)_{p} & =\bigcap_{t \in \mathbb{R}} \operatorname{Ker}\left(\mathrm{d} \psi_{\exp (t \eta)}-I d\right) \\
& =\bigcap_{\theta \in T^{\eta}} \operatorname{Ker}\left(\mathrm{d} \psi_{\theta}-I d\right) \\
& =\mathrm{T}_{p} \operatorname{Fix}\left(T^{\eta}\right)
\end{aligned}
$$

onde a última igualdade foi provada no lema 3.2.21. Isso prova que $\mu^{\eta}$ é função de Morse-Bott.

Lembrando que o grupo d $\psi_{\exp (t \eta)} v$ é unitário com relação a $J_{p}$, pois é Riemanniano com relação a $g_{p}$ e simplético com relação a $\omega_{p}$, seu gerador $\nabla_{(\cdot)} X_{\mu^{\eta}}(p)=-J_{p} \operatorname{Hess}\left(\mu^{\eta}\right)_{p}$ também o é. Logo Hess $\left(\mu^{\eta}\right)_{p}$ comuta com $J_{p}$ e todos os seus autoespaços são unitários, portanto de dimensão par. Assim $\mu^{\eta}$ tem índice e coíndice pares em $p$.

\subsection{Teorema de Schur e Horn}

Vamos agora demonstrar o teorema 3.1.2 introduzido no início do capítulo. Nessa seção faremos uso extensivo de alguns grupos de matrizes. Para propósitos de fixar a notação, vamos relembrar as definições dos grupos que serão usados. Considere $M_{n}(\mathbb{C})$ o grupo das matrizes quadradas de ordem $n$. Dada uma matriz $U \in M_{n}(\mathbb{C})$, definimos sua adjunta $U^{*}$ por $\left(U^{*}\right)_{i j}=\overline{U_{j} i}$. Definimos então o grupo das matrizes unitárias por

$$
U(n)=\left\{U \in M_{n}(\mathbb{C}) \mid U^{*}=U^{-1}\right\}
$$

A álgebra de Lie de $U(n)$ por sua vez coincide com um outro grupo de matrizes chamado grupo das matrizes anti-Hermitianas, dado por

$$
\mathfrak{u}(n)=\left\{u \in M_{n}(\mathbb{C}) \mid u^{*}=-u\right\}
$$

Outro grupo que usaremos é o grupo das matrizes hermitianas, definido por

$$
\mathcal{H}(n)=\left\{H \in M_{n}(\mathbb{C}) \mid H^{*}=H\right\}
$$

Note que multiplicação por $i$ é um isomorfismo entre $\mathfrak{u}(n)$ e $\mathcal{H}(n)$.

Como já foi dito na seção 3.1, o teorema de Schur e Horn é uma aplicação do teorema de convexidade para a ação coadjunta de $U(n)$, que é equivalente à ação de $U(n)$ em $\mathcal{H}(n) \approx \mathfrak{u}(n)^{*}$ por conjugação.

Lembraremos também, que o espectro de uma matriz quadrada $A$ aqui é definido como um vetor $\lambda \in \mathbb{R}^{k}$, onde cada entrada $\lambda_{i}$ é um autovalor de $A$, cada autovalor de $A$ aparece em $\lambda$ um número de vezes igual à dimensão do seu autoespaço associado e os autovalores são ordenados em ordem decrescente. Nesse caso sempre trataremos de espectros de matrizes diagonalizáveis, portanto teremos sempre $\lambda \in \mathbb{R}^{n}$.

Antes da prova do teorema, apresentaremos três lemas. O primeiro não passa de um corolário do teorema espectral para matrizes hermitianas.

Lema 3.3.1. Seja A uma matriz anti-hermitiana. Se A comuta com todas as matrizes $g \in U(n)$, então A é diagonal e as entradas na diagonal de A são números imaginários puros.

Demonstração. Observe primeiro que a matriz $i A$ é hermitiana. Assim, o teorema espectral nos diz que $i A$ é unitariamente diagonalizável, ou seja, existem $\tilde{g} \in U(n)$ e $D$ matriz diagonalcom entradas reais tais que $i A=\tilde{g} D \tilde{g}^{-1}$. Mas isso implica que $D=\tilde{g}^{-1}(i A) \tilde{g}=i A$, logo temos $A=-i D$.

O lema a seguir introduz uma relação entre a ação coadjunta de $U(n)$ e matrizes isoespectrais em $\mathfrak{u}(n) \approx \mathcal{H}(n)$. Esse será o ponto central do teorema que nos permitirá construir uma ação Hamiltoneana na qual aplicar o teorema de convexidade. 
Lema 3.3.2. Seja $U(n)$ o grupo das matrizes unitárias e Ad : $U(n) \times \mathfrak{u}(n) \rightarrow \mathfrak{u}(n)$ sua ação adjunta. Então as órbitas de Ad coincidem com os conjuntos isospectrais de $\mathfrak{u}(n)$.

Demonstração. Como $U(n)$ é um grupo de matrizes, temos que $\operatorname{Ad}(g) A=g A g^{-1}$, logo a ação adjunta preserva espectros, e, se $\mathcal{O}_{\lambda}$ é o conjunto das matrizes de espectro $\lambda$ em $U(n)$, temos $\operatorname{Ad}(U(n)) \subset \mathcal{O}_{\lambda}$.

Por outro lado, dadas $\eta, \tilde{\eta} \in \mathcal{O}_{\lambda}$, o lema 3.3.1 garante que existem $g, \tilde{g} \in U(n)$ e $D, \tilde{D}$ matrizes diagonais tais que $D=g \eta g^{-1}$ e $\tilde{D}=\tilde{g} \tilde{\eta} \tilde{g}^{-1}$. Mas como $\eta$ e $\tilde{\eta}$ tem espectro $\lambda, D$ e $\tilde{D}$ diferem apenas por permutação dos elementos da diagonal, o que pode ser corrigido por conjugação por um elemento de $U(n)$. Assim, sem perda de generalidade, podemos assumir $g \eta g^{-1}=\tilde{g} \tilde{\eta} \tilde{g}^{-1}$, ou equivalentemente, $\tilde{\eta}=\left(\tilde{g}^{-1} g\right) \eta\left(\tilde{g}^{-1} g\right)^{-1}$. Portanto, $\tilde{\eta} \in \operatorname{Ad}(U(n))$. Podemos então concluir que $\operatorname{Ad}(U(n))=\mathcal{O}_{\lambda}$.

O último lema trata de sugrupos agindo pela ação co-adjunta de um grupo. Recorde que vimos, no teorema 2.4.1 que para cada órbita co-adjunta $\omega_{\xi^{*}}\left(X^{\eta^{1}}\left(\xi^{*}\right), X^{\eta^{2}}\left(\xi^{*}\right)\right)=\xi^{*}\left(\left[\eta^{1}, \eta^{2}\right]\right)$ define uma forma simplética e a ação co-adjunta é Hamiltoneana com aplicação momento dada pela inclusão $i_{M}: M \rightarrow \mathfrak{g}^{*}$. O que o lema diz, a grosso modo, é que quando reduzimos a ação adjunta a um subgrupo, a aplicação momento é a projeção ortogonal sobre o dual da álgebra de Lie do subgrupo.

Lema 3.3.3. Seja $G$ grupo de Lie, $\zeta \in \mathfrak{g}^{*}$ e $M=\operatorname{Ad}^{*}(G) \zeta$. Seja também $\langle\cdot, \cdot\rangle$ métrica em $\mathfrak{g} e$ $\theta: \mathfrak{g} \rightarrow \mathfrak{g}^{*}$ o isomorfismo definido por $\theta(\xi)=\langle\xi, \cdot\rangle$. $\theta$ induz uma métrica em $\mathfrak{g}^{*}$, que também chamaremos de $\langle\cdot, \cdot\rangle$. Então, se $H$ subgrupo de Lie de $G$ com álgebra de Lie $\mathfrak{h}, i_{\mathfrak{h}}: \mathfrak{h} \rightarrow \mathfrak{g}$ é a inclusão entre as álgebras de Lie e $\pi_{\theta(\mathfrak{k})}: \mathfrak{g} \rightarrow \theta(\mathfrak{h})$ é a projeção ortogonal sobre $\theta(\mathfrak{h})$, temos que $\left.\mathrm{Ad}^{*}\right|_{H \times M}$ é Hamiltoneana com aplicação momento dada por $i_{\mathfrak{h}}^{*} \circ \pi_{\theta(\mathfrak{h})}$.

Demonstração. Esse resultado foi provado no exemplo 2.3.16.

Finalmente, antes de iniciarmos a prova do teorema, definiremos diag : $M_{n \times n}(\mathbb{C}) \rightarrow \mathbb{C}^{n}$ função que leva matrizes complexas no vetor cujas entradas correspondem às entradas da diagonal da matriz, ou seja, tal que $\operatorname{diag}(A)_{i}=A_{i i}$. Vale relembrar também que, como definido na seção 3.1, dado um vetor $\lambda=\left(\lambda_{1}, \ldots, \lambda_{n}\right) \in \mathbb{R}^{n}$, denotamos

$$
P(\lambda)=\left\{\left(\lambda_{\sigma(1)}, \ldots, \lambda_{\sigma(n)}\right) \mid \sigma \in P(n)\right\}
$$

onde $P(n)$ é o conjunto das permutações de $1, \ldots, n$, e

$$
C(\lambda)=\mathrm{CH}(P(\lambda))
$$

onde $\mathrm{CH}$ denota o casco convexo.

Teorema. Sejam $\lambda, d \in \mathbb{R}^{n}$. Existe $A \in \mathcal{H}(n)$ com espectro $\lambda$ e $\operatorname{diag}(A)=d$ se, e só se, $d \in C(\lambda)$.

Demonstração. Considere $U(n)$ o grupo das matrizes unitárias de ordem $n$, e sua ação co-adjunta $\operatorname{Ad}^{*}: U(n) \times \mathfrak{u}(n)^{*} \rightarrow \mathfrak{u}(n)^{*}$. Seja $\langle\cdot, \cdot\rangle$ métrica bi-invariante em $U(n)$ definida pela fórmula $\langle A, B\rangle=$ $\operatorname{Re} \operatorname{tr}\left(A B^{*}\right)$. Note que se $A, B \in \mathfrak{u}(n)$, temos que $\langle A, B\rangle=-\operatorname{Re} \operatorname{tr}(A B)$.. Podemos então definir o isomorfismo $\theta: \mathfrak{u}(n) \rightarrow \mathfrak{u}(n)^{*}$ dado por $\theta(A)=\langle A, \cdot\rangle$.

Vamos provar que $\theta$ é equivariante com relação a $\mathrm{Ad}_{\mathrm{d}} \mathrm{Ad}^{*}$. De fato, dadas $A, B \in \mathfrak{u}(n)$

$$
\begin{aligned}
\left(\operatorname{Ad}^{*}(g) \circ \theta\right)(A)(B) & =\operatorname{Ad}^{*}(g)(\theta(A))(B) \\
& =\theta(A)\left(\operatorname{Ad}\left(g^{-1}\right) B\right) \\
& =\left\langle A, \operatorname{Ad}\left(g^{-1}\right)\right\rangle \\
& =\langle\operatorname{Ad}(g) A, B\rangle \\
& =(\theta \circ \operatorname{Ad}(g))(A)(B)
\end{aligned}
$$


Logo, podemos usar $\theta$ para transportar os resultados do lema 3.3.3 para $\mathfrak{u}(n)$. Ou seja, se $M=\operatorname{Ad}(U(n)) \eta$ é órbita adjunta de $\mathfrak{u}(n)$, existe $\omega$ forma simplética em $M$ tal que a ação $\left.\operatorname{Ad}\right|_{U(n) \times M}$ é Hamiltoneana com aplicação momento dada por $\left.i_{\theta(M)} \circ \theta\right|_{M}=\theta \circ i_{M}$. Pelo lema 3.3.2 e o fato que $M$ é subconjunto das matrizes anti-hermitianas, todas as matrizes em $M$ tem o mesmo espectro $i \lambda$, onde $\lambda \in \mathbb{R}^{n}$. Logo $-i M$ é o conjunto de matrizes hermitianas de espectro $\lambda$. Se provarmos que $\operatorname{diag}(-i M)=C(\lambda)$, ou equivalentemente, que diag $(M)=i C(\lambda)$, teremos provado o teorema.

Para isso considere $T$ o conjunto das matrizes diagonais em $U(n)$. $T$ é compacto, pois é fechado dentro de um compacto, e é abeliano, logo $T$ é um toro. Se chamarmos $\mathfrak{t}=\operatorname{Lie}(T)$, podemos então fazer a identificação

$$
\begin{array}{ccccc}
\mathfrak{t}^{*} & \approx & \mathfrak{t} & \approx & \mathbb{R}^{n} \\
\langle A, \cdot\rangle & \stackrel{\theta^{-1}}{\longrightarrow} & A & \longrightarrow & \operatorname{diag}(A)
\end{array}
$$

onde $\operatorname{diag}(A)$ é o vetor composto pelas entradas da diagonal de $A$. Note que $\left.\operatorname{diag}\right|_{\mathfrak{t}}: \mathfrak{t} \rightarrow \mathbb{R}^{n}$ é um isomorfismo, pois todas as matrizes $A \in \mathfrak{t}$ são da forma

$$
\left.\frac{\mathrm{d}}{\mathrm{d} t}\left[\begin{array}{ccc}
e^{\lambda_{1} 2 \pi i t} & & 0 \\
& \ddots & \\
0 & & e^{\lambda_{n} 2 \pi i t}
\end{array}\right]\right|_{t=0}=\left[\begin{array}{ccc}
\lambda_{1} & & 0 \\
& \ddots & \\
0 & & \lambda_{n}
\end{array}\right]
$$

Suponha provado que exisite aplicação momento $\mu: M \rightarrow \mathfrak{t}^{*}$ tal que, usando a identificação $\mathfrak{t}^{*} \approx \mathbb{R}^{n}$ acima, $\mu(A)=\operatorname{diag}(A)$. Vamos provar que se $\mathcal{F}$ o conjunto dos pontos fixos de $\left.\operatorname{Ad}\right|_{T \times M}$ então $\mu(\mathcal{F})=i P(\lambda)$. Note que $A \in \mathcal{F}$ se, e só, se $\operatorname{Ad}(g) A=g A g^{-1}=A$, ou seja, $A$ comuta com todas as matrizes diagonais de $U(n)$. Pelo lema 3.3.1, temos que $A$ é diagonal com entradas imaginárias puras na diagonal. Como $A$ é diagonal, $\operatorname{diag}(A)$ é uma permmutação de seu espectro $i \lambda$. Logo, $\operatorname{diag}(\mathcal{F})=i P(\lambda)$. O teorema de convexidade nos garante, então, que $\operatorname{diag}(M)=i C(\lambda)$.

Nos resta apenas provar que existe a aplicação momento desejada. O lema 3.3.3 nos dá uma aplicação momento para $\left.\mathrm{Ad}^{*}\right|_{T \times \theta(M)}$, que podemos transportar via o isomorfismo $\theta$ e obter $\mu=$ $i_{\mathfrak{h}}^{*} \circ \pi_{\theta(\mathfrak{h})} \circ \theta$ como aplicação momento para $\left.\mathrm{Ad}\right|_{T \times M}$. Vamos provar que $\mu$ é a aplicação momento que procuramos, ou seja que diag $\circ \theta^{-1} \circ \mu=\left.\operatorname{diag}\right|_{M}$.

Na prova do lema 3.3.3 mostramos que vale a equação $\theta \circ \pi_{\mathfrak{h}}=\pi_{\theta(\mathfrak{h})} \circ \theta$, o que nos dá

$$
\begin{aligned}
\mu(A) & =i_{\mathfrak{h}}^{*} \circ \pi_{\theta(\mathfrak{h})} \circ \theta(A) \\
& =i_{\mathfrak{h}}^{*} \circ \theta \circ \pi_{\mathfrak{h}}(A) \\
& =\left.\theta\left(\pi_{\mathfrak{h}}(A)\right)\right|_{\mathfrak{t}}
\end{aligned}
$$

Basta provar, então que $\pi_{\mathfrak{t}}$ preserva a diagonal de $A$. Faremos isso mostrando que $\pi_{\mathfrak{t}}(A)=A^{D}$, onde $A^{D}$ é a parte diagonal de $A$. Para isso, note que $\mathfrak{t}$ é gerado pelas matrizes $e_{j}$, onde $\left(e_{j}\right)_{m, n}=$ $i \delta_{j m} \delta_{m n}$, ou seja, matrizes com zero em todas as entradas exceto o $j$-ésimo elemento da diagonal cuja entrada é $i$. Logo

$$
\begin{aligned}
\pi_{\mathfrak{t}}(A) & =\sum_{i=0}^{n}\left\langle A, e_{j}\right\rangle e_{j} \\
& =\sum_{j=0}^{n}-\operatorname{Retr}\left(A, e_{j}\right) e_{j} \\
& =\sum_{j=0}^{n}-i a_{j k} e_{j} \\
& =A^{D}
\end{aligned}
$$




\subsection{Teorema de Delzant}

O teorema de Delzant é um teorema de classificação de variedades tóricas, identificando-as com politopos de Delzant. Vamos começar relembrando rapidamente o conceito de variedades tóricas apresentado na seção 3.1 e definir formalmente politopos de Delzant.

Uma variedade tórica é um $T^{n}$-espaço Hamiltoneano $\left(M, \omega, T^{n}, \mu\right)$ tal que $M$ é $2 n$-dimensional e a ação de $T^{n}$ em $M$ é efetiva.

Um politopo $\Delta$ em $\mathbb{R}^{n}$ é dito de Delzant se ele é simples, racional e suave. Diremos que $\Delta$ é simples se em cada vértice se encontram exatamente $n$ arestas. $\Delta$ será dito racional se, em cada vértice $p$, pudermos escolher $v_{1}, \ldots, v_{n} \in \mathbb{Z}^{n}$ vetores diretores para as arestas que se encontram em $p$. Finalmente, $\Delta$ será suave se, em cada vértice, os $v_{1}, \ldots, v_{n}$ escolhidos na condição anterior formem uma base de $\mathbb{Z}^{n}$.

Os politopos na figura 3.2 todos são politopos de Delzant
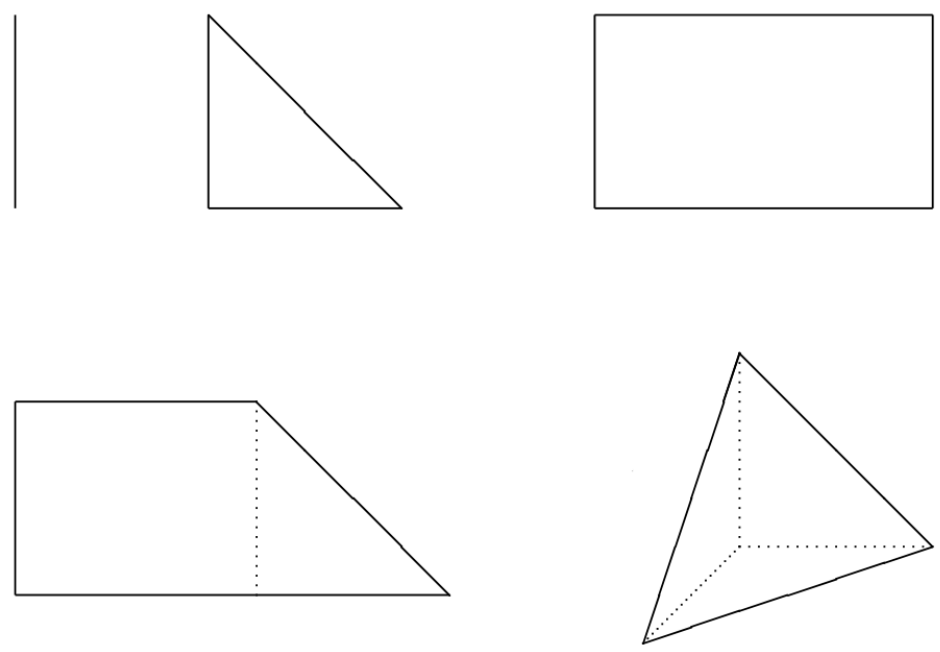

Figura 3.2: Exemplos de politopos de Delzant

Os dois politopos na figura 3.3 não são de Delzant. O politopo da esquerda falha na condição de racionalidade, já que, tanto no vértice de cima quanto no da esquerda, não é possível escolher um sistema de coordenadas tal que os vetores diretores das duas arestas que se encontram estejam em $\mathbb{Z}^{2}$. Já o da direita falha na condição de simplicidade, pois o vértice do topo é o encontro de 4 arestas enquanto os outros tem apenas 3 .
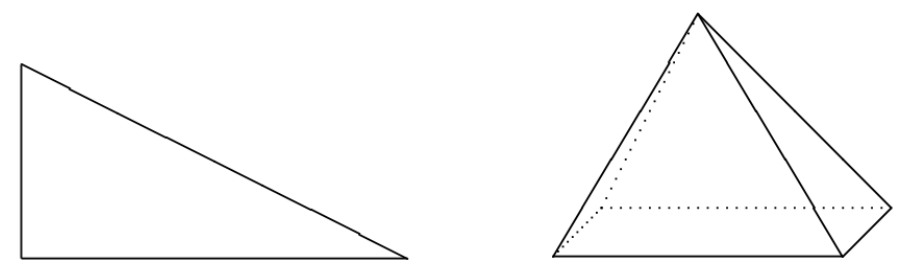

Figura 3.3: Exemplos de politopos não Delzant

As figuras 3.2 e 3.3 acima fora retiradas de [1], Capítulo 28.

Vamos relembrar também o enunciado do teorema de Delzant:

Teorema. Existe uma correspondência um a um entre variedades tóricas e politopos de Delzant. Essa correspondência é dada pela imagem da aplicação momento, ou seja, se $(M, \omega, \psi, \mu)$ é uma variedade tórica, o politopo de Delzant correspondente é $\mu(M)$. 
Esse é um resultado bastante profundo, com uma demosntração usando conceitos bastante abstratos tanto de álgebra quanto de geometria. O que faremos aqui é apenas construir a variedade tórica associada ao politopo $\Delta=[0, a] \subset \mathbb{R}^{*}$. Essa construção é feita baseada em [1], capítulo 29.

Antes de discutirmos o teorema, no entanto, vamos apresentar alguns lemas.

Lema 3.4.1. Sejam $V$ um espaço vetorial dotado de produto interno $\langle\cdot, \cdot\rangle, W$ sub-espaço de $V$ e $i_{W}: W \rightarrow V$ a inclusão. Se $\theta: V \rightarrow V^{*}$ é a aplicação de Riesz definida por $\theta(v)=\langle v, \cdot\rangle, e$ existe um produto interno em $V^{*}$ que faz de $\theta$ isometria. Além disso, $\theta(W)$ é isomorfo a $W^{*}$ via restrição dos funcionais a W. Compondo a projeção ortogonal com essa identificação obtemos a função $\pi: V^{*} \rightarrow W^{*}$. Temos, então, que $\pi=i_{W}^{*}$.

Demonstração. A prova desse lema já foi feita como parte da demonstração do lema 3.3.3. Lá provamos que, dados $\xi \in V^{*}$ e $\eta \in W$, temos $i_{W}^{*}(\xi)(\eta)=\xi(\eta)=\pi \circ \xi(\eta)$.

Note que no lema 3.3.3 $\mathrm{V}$ e $W$ eram álgebras de Lie, mas seus colchetes não foram usados, apenas suas estruturas lineares, logo a demonstração se aplica aqui.

Lema 3.4.2. Seja $(M, \omega)$ variedade simplética e $G, \tilde{G}$ grupos de Lie com álgebras de Lie $\mathfrak{g}$ e $\tilde{\mathfrak{g}}$, respectivamente. Dados $\psi: G \times M \rightarrow M, \tilde{\psi}: \tilde{G} \times M \rightarrow M$ ações e $L: \tilde{G} \rightarrow G$ isomorfismo de grupos tais que $\left(\operatorname{Id}_{M}, L\right)$ é equivariante com relação a $\psi$ e $\tilde{\psi}$, ou seja, que $\psi(L(g), p)=\tilde{\psi}(g, p)$ para todos $g \in \tilde{G}$ e $p \in M$. Equivalentemente, podemos pedir que o diagrama abaixo comute.

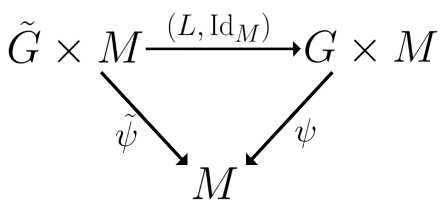

Então, se $\psi$ é ação Hamiltoneana com aplicação momento $\mu: M \rightarrow \mathfrak{g}$, temos que $\tilde{\psi}$ também é Hamiltoneana com aplicação momento dada por $\tilde{\mu}=\left(\mathrm{d} L_{e}\right)^{*} \circ \mu$.

Demonstração. Durante esse lema, denotaremos o campo infinitesimal da ação $\tilde{\psi}$ gerado por $\eta \in \tilde{\mathfrak{g}}$ como $\tilde{X}^{\eta}$. Os campos infinitesimais de $\psi$ seguirão com a notação normal.

O lema será provado se provarmos que, para todo $\eta \in \tilde{\mathfrak{g}}, X_{\tilde{\mu}}^{\eta}=X^{\eta}$. Para isso, observe primeiro que, se $p \in M$, então

$$
\begin{aligned}
\tilde{\mu}^{\eta}(p) & =\tilde{\mu}(p)(\eta) \\
& =\left(\mathrm{d} L_{e}\right)^{*} \circ \mu(f(p))(\eta) \\
& =\mu(p)\left(\mathrm{d} L_{e}(\eta)\right) \\
& =\mu^{\mathrm{d} L_{e}(\eta)}(p)
\end{aligned}
$$

Assim, dados $v_{p} \in \mathrm{T}_{p} \tilde{M}$ e $\alpha:(-\epsilon, \epsilon) \rightarrow \tilde{M}$ tal que $\alpha(0)=p$ e $\alpha^{\prime}(0)=v_{p}$, temos

$$
\begin{aligned}
\omega\left(X_{\tilde{\mu}^{\eta}}(p), v_{p}\right) & =\mathrm{d} \tilde{\mu}_{p}^{\eta}\left(v_{p}\right) \\
& =\left.\frac{\mathrm{d}}{\mathrm{d} t} \tilde{\mu}^{\eta}(\alpha(t))\right|_{t=0} \\
& =\left.\frac{\mathrm{d}}{\mathrm{d} t} \mu^{\mathrm{d} L_{e}(\eta)}(\alpha(t))\right|_{t=0} \\
& =\mathrm{d} \mu^{\mathrm{d} L_{e}(\eta)}\left(v_{p}\right) \\
& =\omega\left(X_{\mu^{\mathrm{d} L_{e}(\eta)}}(p), v_{p}\right) \\
& =\omega\left(X^{\mathrm{d} L_{e}(\eta)}(p), v_{p}\right)
\end{aligned}
$$

Assim, como $\omega$ é não degenerada, temos que $X_{\tilde{\mu}} \eta=X^{\mathrm{d} L_{e}(\eta)}$. Logo basta provar que $X^{\mathrm{d} L_{e}(\eta)}(p)=$ $\tilde{X}^{\eta}(p)$. Note que em cada lado da equação o campo infinitesimal induzido por uma ação diferente. 
Antes disso, no entanto, observe que, se $\exp _{G}$ e $\exp _{\tilde{G}}$ são as aplicações exponenciais de cada grupo, vale que $L \circ \exp _{\tilde{G}}=\exp _{G} \circ \mathrm{d} L$. Logo

$$
\begin{aligned}
X^{\mathrm{d} L_{e}(\eta)}(p) & =\left.\frac{\mathrm{d}}{\mathrm{d} t} \psi\left(\exp _{G}\left(t \mathrm{~d} L_{e}(\eta)\right), p\right)\right|_{t=0} \\
& =\left.\frac{\mathrm{d}}{\mathrm{d} t} \psi\left(L \circ \exp _{\tilde{G}}(t \eta), p\right)\right|_{t=0} \\
& =\left.\frac{\mathrm{d}}{\mathrm{d} t} \tilde{\psi}\left(\exp _{\tilde{G}}(t \eta), p\right)\right|_{t=0} \\
& =\tilde{X}^{\eta}(p)
\end{aligned}
$$

Vamos agora à construção prometida.

Note que a definição de $\Delta$ é um pequeno abuso de notação, associando o politopo com um intervalo de $\mathbb{R}$ via a identificação

$$
\mathbb{R} \ni \lambda \approx \lambda \operatorname{Id}_{\mathbb{R}} \in \mathbb{R}^{*}
$$

Dessa forma, uma maneira mais precisa de representar o politopo seria como o conjunto $\Delta=$ $\left\{x \in \mathbb{R}^{*} \mid 0<x(1)<a\right\}$. Mais adiante, será interessante descrever $\Delta$ como por um conjunto de restrições, e iremos preferir a forma $\Delta=\left\{x \in \mathbb{R}^{*} \mid x(-1)<0, x(1)<a\right\}$.

Considere a ação $\phi: T^{2} \times \mathbb{C}^{2} \rightarrow \mathbb{C}^{2}$ dada por $\psi\left(\left(e^{2 \pi i t_{1}}, e^{2 \pi i t_{2}}\right),\left(z_{1}, z_{2}\right)\right)=\left(e^{2 \pi i t_{1}} z_{1}, e^{2 \pi i t_{2}} z_{2}\right)$. Se usarmos a identificação $\mathbb{C}^{2} \approx \mathbb{R}^{4}$ para usar a forma simplética $\omega=\mathrm{d} x_{1} \wedge \mathrm{d} y_{1}+\mathrm{d} x_{2} \wedge \mathrm{d} y_{2}$ em $\mathbb{C}^{2}$, então, como vimos no exemplo $2.3 .9, \phi$ é uma ação Hamiltoneana com aplicação momento $\nu: \mathbb{C}^{2} \rightarrow\left(\mathbb{R}^{2}\right)^{*}$ dada por

$$
\nu\left(z_{1}, z_{2}\right)\left(t_{1}, t_{2}\right)=-\pi\left(\left(\left|z_{1}\right|^{2}+k_{1}\right) t_{1}+\left(\left|z_{2}\right|^{2}+k_{2}\right) t_{2}\right)
$$

onde $k_{1}, k_{2} \in \mathbb{R}$ são constantes que escolheremos como $k_{1}=0$ e $k_{2}=-\frac{a}{2 \pi}$.

Agora a idéia dessa construção é, a menos de identificações, a seguinte: encontraremos um subgrupo $N$ de $T^{2}$ e faremos a redução para grupos produto como descrita em 3.4 para o produto $T^{2} \approx N \times T^{2} / N$. Denote por $\mathfrak{n}$ e $\mathfrak{t}$ as álgebras de Lie de $N$ e $T=T^{2} / N$, respectivamente, e $i_{\mathfrak{n}}: \mathfrak{n} \rightarrow \mathbb{R}^{2}$ e $i_{\mathfrak{t}}: \mathfrak{t} \rightarrow \mathbb{R}^{2}$ as respectivas inclusões. Então teremos a aplicação momento $\nu$ decomposta em

$$
\nu=i_{\mathfrak{n}}^{*} \circ \nu+i_{\mathfrak{t}}^{*} \circ \nu
$$

A redução de grupos consistirá em aplicar a redução de Marsden em $\mathbb{C}^{2}$ para a ação $\phi$ restrita ao subgrupo $N$, obtendo $M_{\Delta}=\frac{\left(i_{\mathfrak{n}} \circ \nu\right)^{-1}(0)}{N}$. Veremos que $N$ será obtido como o kernel de uma aplicação injetora $\mathfrak{p}: \mathbb{R}^{2} \rightarrow \mathbb{R}$ (onde 2 e 1 foram escolhidos por serem o número de vértices e dimensão do espaço do politopo $\Delta$, respectivamente), e portanto $\operatorname{dim}(N)=1$. Mostraremos que $\left(i_{\mathfrak{n}} \circ \nu\right)^{-1}(0)$ é uma esfera isomorfa a $S^{3}$, e, tomando seu quociente por um grupo unidimensional, obteremos $M_{\Delta}=\mathbb{C P}^{1}$ variedade bidimensional com uma forma simplética induzida $\omega_{\Delta}$.

Para completar a quádrupla $\left(M_{\Delta}, \omega_{\Delta}, T^{1}, \mu\right)$ que forma a variedade tórica, recorde que provamos em 3.4 que a ação $\phi$ restrita ao subgrupo $T^{1} \approx T^{2} / N$ induz uma ação $\psi: T^{1} \times M_{\Delta} \rightarrow M_{\Delta}$ no quociente. Além disso, a aplicação $\left.\left(i_{\mathfrak{t}}^{*} \circ \nu\right)\right|_{\left(i_{\mathfrak{n}}^{*} \circ \nu\right)^{-1}(0)}$ é constante nas órbitas de $N$ e induz $\mu: M_{\Delta} \rightarrow \mathfrak{t}^{*}$ que é aplicação momento para $\psi$.

Vamos agora à construção de fato. Considere a função $\mathfrak{p}: \mathbb{R}^{2} \rightarrow \mathbb{R}$ dada por $\mathfrak{p}\left(t_{1}, t_{2}\right)=t_{2}-t_{1}$. É fácil ver que $\mathfrak{p}$ é sobrejetora e que $\mathfrak{p}\left(\mathbb{Z}^{2}\right)=\mathbb{Z}$. Assim, usando a indentificação $\mathbb{R}^{2} / \mathbb{Z}^{2}=T^{2}$, temos que $\mathfrak{p}$ induz uma aplicação $P: T^{2} \rightarrow T^{1}$, dada por $P\left(e^{2 \pi i t_{1}}, e^{2 \pi i t_{2}}\right)=e^{2 \pi i\left(t_{1}-t_{2}\right)}$.

Defina $N=\operatorname{Ker} P=\left\{\left(e^{2 \pi i t}, e^{2 \pi i t}\right) \mid t \in \mathbb{R}\right\}$ e $\mathfrak{n}=\operatorname{Lie}(N)=\{(t, t) \mid t \in \mathbb{R}\}$. Como já foi mostrado no lema 2.3.15, se $i_{\mathfrak{n}}: \mathfrak{n} \rightarrow \mathbb{R}^{2}$ é a inclusão, então $i_{\mathfrak{n}}^{*} \circ \nu$ é aplicação momento para a ação $\left.\phi\right|_{N \times \mathbb{C}^{2}}$.

Desejamos fazer a redução simplética de $\mathbb{C}^{2}$ pela ação de $\phi_{N \times \mathbb{C}^{2}}$. Considere então o nível zero $Z=\left(i_{\mathfrak{n}}^{*} \circ \nu\right)^{-1}(0)$. Vamos mostrar que $N$ age livremente em $Z$. Para isso vamos obter uma descrição 
mais precisa do conjunto $Z$. Observe que, dados $\left(z_{1}, z_{2}\right) \in \mathbb{C}^{2}$ e $(t, t) \in \mathfrak{n}$, então

$$
\begin{aligned}
\left(i_{\mathfrak{n}}{ }^{*} \circ \nu\right)\left(z_{1}, z_{2}\right)(t, t) & =i_{\mathfrak{n}}{ }^{*}\left(\nu\left(z_{1}, z_{2}\right)\right)(t, t) \\
& =\nu\left(z_{1}, z_{2}\right)\left(i_{\mathfrak{n}}(t, t)\right) \\
& =\pi\left(\left(\left|z_{1}\right|^{2}-\frac{a}{2 \pi}\right) t+\left|z_{2}\right|^{2} t\right) \\
& =\pi\left(\left|z_{1}\right|^{2}+\left|z_{2}\right|^{2}-\frac{a}{2 \pi}\right) t
\end{aligned}
$$

Logo $\left(i_{\mathfrak{n}}{ }^{*} \circ \nu\right)\left(z_{1}, z_{2}\right)=0$ se, e só se, $\left|z_{1}\right|^{2}+\left|z_{2}\right|^{2}=\frac{a}{2}$. Isso nos dá

$$
Z=\left\{\left.\left(z_{1}, z_{2}\right) \in \mathbb{C}^{2}|| z_{1}\right|^{2}+\left|z_{2}\right|^{2}=\frac{a}{2}\right\}
$$

e $Z$ é a esfera de raio $\frac{a}{2}$ em $\mathbb{C}^{2}$. Como a ação de $T^{2}$ preserva $\left|z_{1}\right|^{2}$ e $\left|z_{2}\right|^{2}$ qualquer subgrupo age livremente sobre $Z$.

Além disso, 0 é valor regular de $i_{\mathfrak{n}}^{*} \circ \nu$. De fato, se escrevermos $\left(z_{1}, z_{2}\right)=\left(x_{1}+i y_{1}, x_{2}+i y_{2}\right)$, então a matriz que representa $\mathrm{d}\left(i_{\mathfrak{n}}^{*} \circ \nu\right)_{\left(z_{1}, z_{2}\right)}$ é

$$
\left[\begin{array}{l}
2 x_{1} \\
2 y_{1} \\
2 x_{2} \\
2 y_{2}
\end{array}\right]
$$

logo o único ponto crítico de $i_{\mathfrak{n}}^{*} \circ \nu$ é $(0,0) \notin Z$.

Assim, o teorema 2.5.5 de redução simplética garante que existe $\omega_{\Delta}$ forma simplética na variedade $M_{\Delta}=Z / N$ tal que, $p^{*} \omega_{\Delta}=i_{Z}^{*} \omega$, onde $p: Z \rightarrow M_{\Delta}$ é a projeção usual sobre o quociente e $i_{Z}: Z \rightarrow \mathbb{C}^{2}$ é a inclusão. Note que $M_{\Delta}$ é o quociente de uma esfera em $\mathbb{C}^{2}$ por uma ação de $S^{1}$, $\operatorname{logo} M_{\Delta}=\mathbb{C P}^{1}$.

Vamos agora mostrar que existe ação Hamiltoneana de $T^{1} \mathrm{em} M_{\Delta}$ com aplicação momento $\mu: M_{\Delta} \rightarrow \mathbb{R}^{*}$ tal que $\mu(\Delta)=\Delta$. Note, no entanto, que o grupo $\tilde{N}=\left\{\left(e^{2 \pi i t}, e^{-2 \pi i t}\right) \mid t \in \mathbb{R}\right\}$ gerado por $\mathfrak{n}^{\perp}$ é um toro. De fato, $\tilde{N}$ é abeliano, pois $T^{2}$ é abeliano, e fechado, pois dada $\left\{g_{n}\right\}_{n \in \mathbb{N}}$ seqüência em $\tilde{N}$ que converge para um elemento $g \in T^{2}$, podemos encontrar $t_{n}$ seqüência em $\mathbb{R}$ que converge para $t \in \mathbb{R}$, tal que $g_{n}=\left(e^{2 \pi i t_{n}}, e^{-2 \pi i t_{n}}\right)$. Logo $g_{n} \rightarrow\left(e^{2 \pi i t}, e^{-2 \pi i t}\right) \in \tilde{N}$.

Como $\operatorname{dim}(\tilde{N})=1$, temos que $\tilde{N} \approx T^{1}$. A idéia desta parte da prova então será encontrar um isomorfismo entre os dois grupos que induza a ação desejada.

Se aplicarmos aqui os resultados da proposição sobre redução para grupos produto, obteremos uma ação hamiltoneana $\tilde{\psi}: \tilde{N} \times M_{\Delta} \rightarrow M_{\Delta}$ com aplicação momento $\tilde{\mu}: M_{\Delta} \rightarrow \mathfrak{n}^{\perp}$ tal que o diagrama abaixo comuta, onde $\pi_{2}:\left(\mathbb{R}^{2}\right)^{*} \rightarrow\left(\mathfrak{n}^{\perp}\right)^{*}$ é a projeção ortogonal pela métrica induzida em $\left(\mathbb{R}^{2}\right)^{*}$ pela métrica canônica de $\mathbb{R}^{2}$.

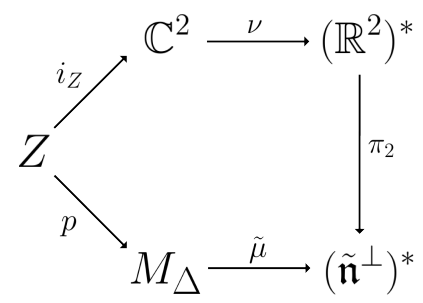

Considere $L: T^{1} \rightarrow \tilde{N}$ definido por $L\left(e^{2 \pi i t}\right)=\left(e^{-2 \pi i t}, e^{2 \pi i t}\right)$. $L$ é um isomorfismo de grupos. A ação $\psi: T^{1} \times M_{\Delta} \rightarrow M_{\Delta}$ definida por $\psi(g, p)=\tilde{\psi}(L(g), p)$ faz com que $\left(\operatorname{Id}_{M_{\Delta}}, L\right)$ seja equivariante. Pelo lema 3.4.2, $\psi$ é Hamiltoneana com aplicação momento dada por $\mu=\mathrm{d} L^{*} \circ \tilde{\mu}$. Podemos então reescrever o diagrama acima como 


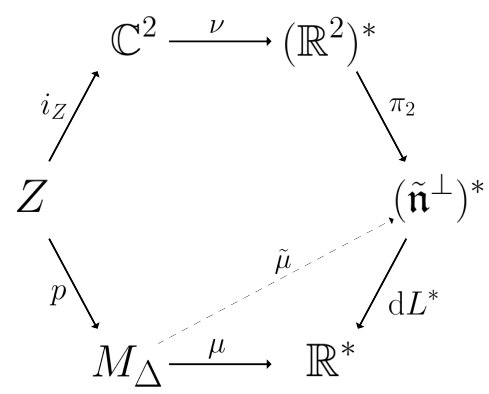

Nos resta apenas provar que $\mu\left(M_{\Delta}\right)=\Delta$.

A comutatividade do segundo diagrama é equivalente à equação

$$
\mu \circ p=\mathrm{d} L^{*} \circ \pi_{2} \circ \nu \circ i_{Z}
$$

Temos então que

$$
\begin{aligned}
\mu\left(M_{\Delta}\right) & =\mu \circ p(Z) \\
& =\mathrm{d} L^{*} \circ \pi_{2} \circ \nu(Z) \\
& =\mathrm{d} L^{*} \circ i_{\mathfrak{n}^{\perp}}^{*} \circ \nu(Z)
\end{aligned}
$$

onde a última igualdade é apenas a aplicação do lema 3.4.1.

Para continuar, vamos provar a igualdade $\nu(Z)=\frac{1}{2} \mathfrak{p}^{*}(\Delta)$. Essa igualdade pode parecer estranha a princípio, mas note que $\nu$ e $\mathfrak{p}$ contém toda a informação de $\Delta$. Isso é porque $\Delta$ é definido pelas restrições $x(-1)<0$ e $x(1)<a$ e usamos os escalares 0 e $a$ para definir as constantes $k_{1}$ e $k_{2}$ em $\nu$ e definimos $\mathfrak{p}$ de forma que $\mathfrak{p}\left(e_{1}\right)=-1$ e $\mathfrak{p}\left(e_{2}\right)=1$. Ao final dessa prova discutiremos como usar essas informações para extender a prova para casos de dimensão maior. Enfim, à prova.

Lembrando que $Z=\left\{\left.\left(z_{1}, z_{2}\right) \in \mathbb{C}^{2}|| z_{1}\right|^{2}+\left|z_{2}\right|^{2}=\frac{a}{2}\right\}$ temos que $\left(z_{1}, z_{2}\right) \in Z$ se, e só se $\left|z_{1}\right|^{2}-\frac{a}{2}=-\left|z_{2}\right|^{2}$, e substituindo na fórmula de $\nu$ temos que

$$
\begin{aligned}
\nu\left(z_{1}, z_{2}\right)\left(t_{1}, t_{2}\right) & =\left(-\left|z_{2}\right|^{2}\right) t_{1}+\left|z_{2}\right|^{2} t_{2} \\
& =\left|z_{2}\right|^{2}\left(t_{2}-t_{1}\right)
\end{aligned}
$$

onde $0 \leq\left|z_{2}\right|^{2} \leq \frac{a}{2}$

Por outro lado, note que se $\lambda \operatorname{Id}_{\mathbb{R}} \in \Delta$, então

$$
\begin{aligned}
\mathfrak{p}^{*}\left(\lambda \operatorname{Id}_{\mathbb{R}}\right)\left(t_{1}, t_{2}\right) & =\lambda \operatorname{Id}_{\mathbb{R}}\left(\mathfrak{p}\left(t_{1}, t_{2}\right)\right) \\
& =\lambda\left(t_{2}-t_{1}\right)
\end{aligned}
$$

onde $0 \leq \lambda \leq a$. Assim provamos $\nu(Z)=\frac{1}{2} \mathfrak{p}^{*}(\Delta)$ e temos que $\mu\left(M_{\Delta}\right)=\mathrm{d} L^{*} \circ i_{\mathfrak{n}^{\perp}}^{*} \circ \frac{1}{2} \mathfrak{p}^{*}(\Delta)$. Dados $x \in \Delta$ e $t \in \mathbb{R}$

$$
\begin{aligned}
\mathrm{d} L^{*} \circ i_{\mathfrak{n} \perp}^{*} \circ \frac{1}{2} \mathfrak{p}^{*}(x)(t) & =\left(\frac{1}{2} \mathfrak{p} \circ i_{\mathfrak{n} \perp} \circ \mathrm{d} L\right)^{*}(x)(t) \\
& =x\left(\frac{1}{2} \mathfrak{p} \circ i_{\mathfrak{n} \perp} \circ \mathrm{d} L(t)\right) \\
& =x\left(\frac{1}{2} \mathfrak{p}(-t, t)\right) \\
& =x(t)
\end{aligned}
$$

$\operatorname{Logo} \mathrm{d} L^{*} \circ i_{\mathfrak{n} \perp}^{*} \circ \frac{1}{2} \mathfrak{p}^{*}=\operatorname{Id}_{\mathbb{R}^{*}}$ e $\mu\left(M_{\Delta}\right)=\Delta$.

Vamos agora discutir como generalizar isso para um politopo de dimensão maior. Vamos primeiro olhar com cuidado a construção acima. Chamaremos de $n$ a dimensão do espaço que contém $\Delta$, ou seja, 1 . Chamaremos de $d$ o número de restrições necessárias para definir $\Delta$, no caso 2 . Esses valores são usados em vários pontos da construção. Vamos dar um novo exemplo, mas dessa vez daremos 
apenas os primeiros passos, já que os seguintes erão iguais aos acima.

Considere agora $\Delta^{\prime}$ o triângulo retângulo isóceles abaixo

Podemos definir $\Delta$ com as seguintes restrições

$$
\begin{aligned}
\Delta=\left\{x \in\left(\mathbb{R}^{2}\right)^{*} \mid\right. & x(1,1)<1 \\
x(1,0) & <-1 \\
x(0,1) & <-1\}
\end{aligned}
$$

Para futuras refrências, sejam $v_{1}=(1,1), v_{2}=(1,0), v_{3}=(0,1), \lambda_{1}=1$ e $\lambda_{2}=\lambda_{3}=-1$. Temos aqui então $n=2$ e $d=3$. O valor de $d$ define quantas cópias de $\mathbb{C}$ irão compor o o espaço inicial e a dimensão do toro agindo nele. Logo, seja $\psi: T^{3} \times \mathbb{C}^{3} \rightarrow \mathbb{C}^{3}$ ação semelhante à dada acima, em que cada componente de $T^{3}=\left(S^{1}\right)^{3}$ age em uma componente de $\mathbb{C}^{3}$ por multiplicação. Essa ação é hamiltoneana com aplicação momento $\nu: \mathbb{C}^{3} \rightarrow\left(\mathbb{R}^{3}\right)^{*}$ definida por

$$
\nu\left(z_{1}, z_{2}, z_{3}\right)\left(t_{1}, t_{2}, t_{3}\right)=-\pi \sum_{i=0}^{3}\left(\left|z_{i}\right|^{2}+k_{i}\right) t_{i}
$$

onde escolheremos $k_{i}=-\lambda_{i}$.

Usaremos agora $d$ e $n$ para determinar a dimensão de domínio e contra-domínio da função $\mathfrak{p}$, respectivamente. Definiremos então $\mathfrak{p}: \mathbb{R}^{3} \rightarrow \mathbb{R}^{2}$ de forma que $\mathfrak{p}\left(e_{i}\right)=v_{i}$. A partir daqui a construção segue como feita acima. Mais especificamente, teremos $N=\operatorname{Ker}(\mathfrak{p})$ unidimensional e $\left(i_{\mathfrak{n}}^{*} \circ \nu\right)^{-1}(0) \approx S^{5}$. Isso nos dá $M_{\Delta}=S^{5} / N=\mathbb{C P}^{2}$, com uma ação Hamiltoneana de $T^{3} / N \approx T^{2}$ com aplicação momento induzida por $\left.\left(i_{\mathfrak{t}}^{*} \circ \nu\right)\right|_{\left(i_{\mathfrak{t}}^{*} \circ \nu\right)^{-1}(0)}$, onde $\mathfrak{t}$ é a álgebra de Lie de $T^{3} / N$ e $i_{\mathfrak{t}}: \mathfrak{t} \rightarrow \mathbb{R}^{3}$ é a inclusão. 


\section{Referências Bibliográficas}

[1] CANNAS, A. Lectures on Symplectic Geometry. Berkeley: Springer-Verlag, 2006. $217 \mathrm{p}$.

[2] HOLM D. D., SCHAMAH T., STOICA C., Geometric Mechanics and Symmetry: From Finite to Infinite Dimensions. Oxford tests in apllied and engineering mathematics

[3] MCDUFF, D. SALAMON, D. Introduction to Symplectic Topology. Segunda Edição. Oxford: Clarendon Press, 1998. 486 p.

[4] BRIQUet, R., RRibeiro, D. N., CASTro, F. I., AlexandrinO, M. M., Rudimentos de Mecânica em Geometria Diferencial, Rio de Janeiro, 2005

[5] THORBERGSSON, G. Symplektische Geometrie I: Eine Vorlesung gehalten im SS 1998 an der Universität zu Köln

[6] http://www-personal.umich.edu/ wangzuoq/437W13

[7] http://www.math.sunysb.edu/ basu/notes/GSS6.pdf

[8] M.F. Atiyah, Convexity and commuting Hamiltonians. Bull, London Math. Soc., 14 (1982), pp. 1-15

[9] Delzant, T., Hamiltoniens periodiques et images convexes de l'application moment, Bull. Soc. Math. France 116 (1988), 315-339

[10] http://www.math.uchicago.edu/ may/VIGRE/VIGRE2010/REUPapers/Schmaltz.pdf 